Final Report

\title{
RADIATION-INDUCED TOPOLOGICAL DISORDER IN IRRADIATED NETWORK STRUCTURES
}

\author{
DOE Grant DE-FG02-89ER45396
}

for the period 22 June 1996 to 21 June 2002

\author{
submitted by \\ Linn W. Hobbs, Principal Investigator \\ Professor of Materials Science \\ Department of Materials Science \& Engineering \\ Massachusetts Institute of Technology \\ Room 13-4054, 77 Massachusetts Avenue \\ Cambridge, MA 02139-4307 \\ Tel: (617) 253-6835 \\ Fax: (617) 252-1020 \\ <hobbs@mit.edu> \\ submitted to \\ Division of Materials Sciences \\ Office of Energy Research \\ U. S. Department of Energy \\ Washington, D.C. 20545 \\ Tel: (301) 903-8230/903-3427 \\ Fax: (301) 903-9513 \\ $<$ Robert.Hwang@science.doe.gov>
}

December 2002 


\section{Summary}

This report summarizes results of a research program investigating the fundamental principles underlying the phenomenon of topological disordering in a radiation environment. This phenomenon is known popularly as amorphization, but is more formally described as a process of radiation-induced structural arrangement that leads in crystals to loss of long-range translational and orientational correlations and in glasses to analogous alteration of connectivity topologies. The program focus has been on a set compound ceramic solids with directed bonding exhibiting structures that can be described as networks. Such solids include $\mathrm{SiO}_{2}, \mathrm{Si}_{3} \mathrm{~N}_{4}, \mathrm{SiC}$, which are of interest to applications in fusion energy production, nuclear waste storage, and device manufacture involving ion implantation or use in radiation fields. The principal investigative tools comprise a combination of experimental diffraction-based techniques, topological modeling, and molecular-dynamics simulations that have proven a rich source of information in the preceding support period.

The results from the present support period fall into three task areas. The first comprises enumeration of the rigidity constraints applying to i) more complex ceramic structures (such as rutile, corundum, spinel and olivine structures) that exhibit multiply polytopic coordination units or multiple modes of connecting such units, ii) elemental solids (such as graphite, silicon and diamond) for which a correct choice of polytope is necessary to achieve correct representation of the constraints, and iii) compounds (such as spinel and silicon carbide) that exhibit chemical disorder on one or several sublattices. With correct identification of the topological constraints, a unique correlation is shown to exist between constraint and amorphizability which demonstrates that amorphization occurs at a critical constraint loss. The second task involves the application of molecular dynamics (MD) methods to topologically-generated models of amorphized network silicas. These methods are shown to generate fully connected topologically-disordered networks, equilibrated to achieve accurately-specified atomic coordinates that can be compared to correlation data derived from diffraction experiments. The MD equilibrations demonstrate the insensitivity of diffraction methods to substantial differences in intermediate-range topology, with the exception of the first diffraction peak which is shown to be uniquely sensitive to topological differences. The third task concerns application of MD simulations to amorphization of silicon carbide, which exhibits anomalous amorphizability. Amorphization of this compound is shown to derive from its facility for tolerating chemical disorder, and a critical homonuclear bond density threshold is established as a criterion for its amorphization. 


\section{TABLE OF CONTENTS}

Summary

1. INTRODUCTION

2. RIGIDITY CONSTRAINTS IN THE AMORPHIZATION OF IRRADIATED COMPLEX CERAMICS

2.1 Multiply-Polytopic Multiply-Connected Structures 8

2.1.1 Rutile

2.1.2 Corundum

2.1.3 Spinel

2.1.4 Olivine

2.2 Polytope Choice in Elemental Networks

2.2.1 Graphite

2.2.2 Diamond and Silicon

2.3 Chemical Disorder

2.3.1 Spinel

2.3.2 Silicon Carbide

2.4 Functional Correlation of Amorphizability and Topological Freedom 14

3. MOLECULAR DYNAMICS MODELING OF AMORPHIZED SILICAS 15

$\begin{array}{ll}3.1 \text { Assembly and Reassembly of Networks } & 15\end{array}$

3.1.1 Amorphous Constructions 16

$\begin{array}{ll}3.1 .2 \text { Reassembly } & 18\end{array}$

3.2 Molecular Dynamics Refinement of Simulated Pseudo-Cascades 18

3.2.1 Initial configurations and potentials 19

3.2.2 Reassembly Topologies

21

3.2.3 Real- and Reciprocal-Space Corrrelations 22

4. MOLECULAR DYNAMICS MODELING OF AMORPHIZED SiC 23

4.1 Topology of SiC 23

4.1.1 Rigidity and Chemical Disorder 24

4.1.2 Chemical and Topological Disorder Metrics 25

4.2 Molecular Dynamics Simulations of Disorder 26 
4.2.1 Choice of Potential 26

4.2.2 Pseudo-Cascade Embedded Simulations 28

4.2.3 Chemical Disorder Simulations 30

4.3 The Amorphizing Transition in SiC 32

5. ON-GOING RESEARCH IN AMORPHIZED STRUCTURES 33

5.1 Characterization of Topological Disorder by 33 Electron Diffraction and Imaging

5.1.1 First Peak in Electron Diffraction 33

5.1.2 Fluctuation Microscopy of Amorphized $\mathrm{SiO}_{2}$, $\mathrm{SiC}$ and $\mathrm{Si}_{3} \mathrm{~N}_{4}$

5.2 Further MD Simulation of SiC Amorphization 36

5.2.1 Ab-initio MD Modeling of Silcon Carbide Structure 36

5.3 MD Simulation of Silcon Nitride Amorphization 38

5.3.1 Topology of $\mathrm{Si}_{3} \mathrm{~N}_{4}$

5.3.2 Potentials for Silicon Nitride $\quad 39$

5.3.3 Topological Analysis of MD-Refined Silicon Nitride 40

5.3.4 Modeling Aluminum Nitride Amorphization 41

5.4 Structural Simulations of Amorphous Silica 41

5.4.1 Paracrystallinity in Amorphous Silicas 41

5.4.2 Ring-Circuit Enumeration in Local Ring Clusters 42

5.4.3 MD Simulation of Silica Anomaly using a Topological Approach 43

5.5 Topological-MD Modeling of Irradiation Amorphized Silicon 44

5.5.1 Topological-MD Simulation of Amorphous Zones in Silicon $\quad 44$

References 45

Publications and Presentations during final Grant Period 58 


\section{INTRODUCTION}

This report summarizes the results of a program of research funded under DOE Grant DE-FG02-89ER45396 for the period June 1996 to June 2002, with the emphasis on the last three-year funding period, 22 June 1999 to 21 June 2002. The program has had as its objectives investigation of the responses to radiation environments of ceramics heavily-irradiated with electrons, neutrons and ions, with potential application to fusion energy technology and high-level nuclear waste storage. The radiation responses of many of these ceramic materials, and non-metals in general, have been reviewed by Clinard and Hobbs [1] and more recently by Hobbs et al. [2]. The major issues involved have been the subject of a series of DOE-sponsored workshops in which the principal investigator has participated. Four most recent have focused on SiC-based composites for fusion energy applications [3], ceramic nuclear waste storage in glass [4] and ceramic [5] media, and amorphization of ceramics [6].

The major focus of the most recent funding period has been on the radiationinduced transformation in ceramic solids from initially crystalline to eventually aperiodic states-states classed geologically as metamict states [7] and a transformation known more popularly as amorphization. For reasons advanced later, these states will henceforth be referred to as topologically disordered [8], implying loss of both translational and orientational long-range correlations. In contrast to metals, only one class of which (ordered alloys) amorphizes in response to disordering radiation [9], many ceramic compounds exhibit a metamict response. The criteria appear to be at least partially topological [8] and rooted in the concepts of structural freedom [10-13] and percolation rigidity [14]. The major technological concerns about amorphization are dimensional stability (e.g., quartz $\left(\mathrm{SiO}_{2}\right)$ [15], zircon $\left(\mathrm{ZrSiO}_{4}\right]$ [16] and many other silicates undergo volume expansion of $5-15 \%$ during amorphization) and increased chemical reactivity (e.g., in nuclear wasteforms, the lowered resistance to leaching [17] that is often exacerbated by microfracturing attendant upon the large volume change). Dimensional stability is critical in certain microstructural configurations, such as SiCfiber/SiC-matrix composites proposed for first wall and blanket applications nuclear fusion reactors.

The scientific interest is in the way solids are disordered by the fluence of displacive radiation and subsequently rearrange and the way atoms order in other than crystalline arrangements. The usual mode of radiation disordering is displacement of atoms in overlapping ballistic collision cascades [18] engendered by fast neutron or medium-to-heavy ion irradiation. Amorphization by single ballistic displacements is also known to occur, e.g. by fast electron irradiation of quartz [19], SiC [20] and ordered metal alloys [9, 21], and by radiolytic displacment mechanisms in silica [22] and silicates [23]. The distinction is important because, in the case of single ballistic or radiolytic displacements, order is lost incrementally, bond-by-bond, and in many solids restorative back reactions, point-defect aggregation or decomposition will occur as alternative responses under these conditions. The metamict transformation in readily amorphizable solids is functionally complete at displacement densities $<1$ displacements per atom (dpa), a radiation dose 1-2 orders of magnitude lower than encountered in other major radiation damage phenomena such as void swelling. Metamict solids tend to recrystallize 
when heated, epitaxially if a crystalline interface is available [24,25], and recrystallize as fast as they disorder during irradiation above a critical amorphization temperature $[26,27]$. The critical amorphization temperature $T_{c}$ may be very high (as high as the 1140 $\mathrm{K} \alpha-\beta$ transition in quartz [28]), very low (probably $<100 \mathrm{~K}$ for $\mathrm{Al}_{2} \mathrm{O}_{3}$ and effectively 0 $\mathrm{K}$ for redundantly constrained structures like $\mathrm{MgO}$ or spinels [27]), or (inconveniently) not far from room temperature, as in $\mathrm{SiC}(<390 \mathrm{~K}$ [29]).

Glasses also lack both translational and orientation order, though it is inaccurate to refer to metamict solids as glasses, because a glass transition may not necessarily exist $[8,30]$. Irradiated glasses "amorphize" too, in the sense that irreversible structural alterations accompany the radiation-induced displacements, leading to terminally altered arrangements which have been mostly characterized by correlative changes in density and refractive index [31]. There is no unique "amorphous" phase of any compound, however, but instead a large range of possible topologically disordered arrangements whose structures depend on their mode of formation [32], some of which are variously accessible to displacive radiation pathways. Vitreous silica appears to be the reproducibly average product of the quenching of characteristic atomic arrangements existing in silica liquid [30]; metamict silica (which is $2.7 \%$ denser than vitreous silica [15]) is the likewise reproducibly average product of rearranging a wide family of crystalline or vitreous $\mathrm{SiO}_{2}$ precursors through bond breakage and rebonding [33], though details of the structure have been shown [34] to depend on which precursor and the mode of irradiative disordering.

Three fundamental questions can be asked about metamictization from either crystalline or glassy precursors: 1) Why are some structures more susceptible to amorphization than others? 2) What are the atomic sequences occurring during the transformation? 3) What is (are) the structure(s) of the metamict state(s)? Work by us on topological criteria (reported in \$2), and by others [10-12,35,36] using combined chemical and structural parameter combinations reflecting dynamic crystallization potential have provided substantial insight into the first question regarding amorphizability. Few studies until recently [37-41] have addressed the disordering signatures of displacive radiation events or the subsequent atomic-scale reconstructions in ceramic solids. And, despite increasingly sophisticated experimental methods brought to bear on radiation-induced amorphization mechanisms in ceramics [42], very little still is known about the atomic structures of the metamict states themselves $[5,43]$.

Historically, our previous programs have divided between experimental and modeling investigations of radiation-induced structural rearrangements in crystalline and glassy ceramic solids. The modeling effort has been concerned with developing a unified topological point of view from to which to understand the structures of crystalline and non-crystalline solids alike, through a focus on the connectivity of structural units. This successful effort has resulted in a demonstrable criterion for amorphizability based on rigidity considerations [10-12] and a unique topological description of a family of network structures $\left(\mathrm{SiO}_{2}, \mathrm{Si}_{3} \mathrm{~N}_{4}, \mathrm{SiC}\right)$ based on closed-circuit connectivities [44]. The experimental portion has focused on the information derivable from fast electron scattering, utilizing inelastic scattering to provide information about local environments through electron energy-loss near-edge fine structure, and elastic scattering to provide information about atom arrangements at intermediate range through energy-filtered electron diffraction and its Fourier transformation into radial density functions. 
The most recent three-year program (summarized in $\S \S 2-4$ ) has focused almost exclusively on topological and molecular dynamics (MD) modeling of the metamict transition and state in $\mathrm{SiO}_{2}$ and $\mathrm{SiC}$, to better understand the topological options available for non-crystalline arrangements, to provide accurate coordinates for topological models for comparison to diffraction data by equilibrating models to realistic potentials, and-in the case of $\mathrm{SiC}$ - to explore the role of chemical disorder in effecting metamict arrangements and amorphizability. The more restictive emphasis was in part due to constriction of the research funds available from DOE and in part due to advantageous utilization of personnel resources as they have serendipitously appeared. This effort has been reported in two extensive book chapters $[45,46]$ and in other individual publications reported in the Appendix.

An important finding of the most recent program-that of all the information derivable from diffraction data, only the first diffraction peak is capable of distinguishing between vastly different topological arrangements, and thus of providing definitive information about intermediate-range arrangements-has important implications for the experimental realm for investigators of amorphous states of material, as they seek to understand the information contained in the diffraction spectrum from amorphized structures. Similarly, the successful MD modeling of $\mathrm{SiO}_{2}$ topological arrangements holds lessons for extension of modeling efforts to more complex silicate structures involving other cations ( $\mathrm{Na}, \mathrm{Ca}, \mathrm{Zr}$ ) for which satisfactory atomic potentials $[47,48]$ have been developed and also into the elemental silicon network, regarding which there is a current interest in radiation-induced degradation of microelectromechanical (MEMS) devices [49]. The latter effort has been embarked upon with the assistance of Professor Martin Bazant, recently arrived from Harvard, who has recently developed a new coordination-affected potential for Si [50]. And lastly, a major extension of the present MD modeling effort in SiC has engaged the collaboration of Professor Sidney Yip of MIT's Nuclear Engineering Department, whose group has carried out extensive MD modeling of thermomechanical properties of, point defects in, and pressure-induced amorphization of SiC [51-55].

\section{RIGIDITY CONSTRAINTS IN THE AMORPHIZATION OF IRRADIATED COMPLEX CERAMICS}

We have shown previously that the ease with which ceramic structures amorphize under displacive radiation can be related to connectivity and the consequent topological constraints which impose rigidity on the original atomic arrangement [10-12]. The starting point is to consider, as connected structural units (polytopes), elemental (M) or cation coordination polyhedra (e.g. $\mathrm{M}-\mathrm{M}$ rods, $\mathrm{M}-\mathrm{M}_{4}$ tetrahedra, $\mathrm{M}-\mathrm{X}_{3}$ triangles, $\mathrm{M}-\mathrm{X}_{4}$ tetrahedra, $\mathrm{M}-\mathrm{X}_{6}$ octahedra, etc.), which are connected by vertex, edge or face sharing. The difference between the degrees of freedom and the structural constraints imposed by connections to neighboring polytopes is known as topological freedom $f$, which can be calculated for simple structures using the formulation of Gupta [56]

$$
f=d-C\{d-[\delta(\delta+1) / 2 V]\}-(d-1)(Y / 2)-[(p-1) d-(2 p-3)](Z / p)
$$


where $d$ is the dimensionality of the structure $(d=1-3), C$ is the number of polytopes of dimensionality $\delta$ common to a vertex, $V$ is the number of vertices per polytope (hence $\{\mathrm{C}, \mathrm{V}\}$ expresses the connectivity), $Y$ is the fraction of edge-sharing vertices and $Z$ the fraction of vertices sharing $p$-sided faces. This parameter has been shown to be generally well correlated (Table 1) to the critical displacement energy density (e.g., eV/atom) or displacement density (dpa) for amorphization in simple ceramic structures (e.g. rocksalt [57], fluorite [58], $\mathrm{Si}_{3} \mathrm{~N}_{4}$ networks [64], $\mathrm{SiO}_{2}$ networks [68]) that have a single polytope form (octahedra for rocksalt, cubes for fluorite, tetrahedra for the last two tetrahedral networks) and a single connectivity mode (edge sharing for the first two, vertex sharing for the last two).

\subsection{Multiply-Polytopic Multiply-Connected Structures}

Structures which have more than one connectivity mode (e.g. vertex and edge sharing in rutile, edge sharing and face sharing in corundum) or more than one polytope type (e.g. tetrahedra and octahedra in spinels or olivine) require a more detailed evaluation than is provided by simple averaging of $C, \delta, Y, p$ and $Z$, if the actual constraints are to be properly evaluated. Elemental structures, like those of $\mathrm{C}$ or $\mathrm{Si}$, can be considered using $\mathrm{M}-\mathrm{M}$ rod polytopes, but these do not capture the special bonding constraint imposed by covalent bonding (in the case of $\mathrm{C}$ and Si by their hybridized trigonal $\mathrm{sp}^{2}$ or tetrahedral $\mathrm{sp}^{3}$ bonding orbitals). In these cases, choice of an alternative polytope which reflects the bonding geometry is required to reflect the overall constraint. For structures such as $\mathrm{SiC}$, for which chemical disorder ( $\mathrm{Si}$ on $\mathrm{C}$ sites, and vice versa) is energetically acceptable, the influence of the possibility and state of chemical disorder on the choice of polytope must be considered. We have devised appropriate evaluation schemes for these more complicated situations, which are reported in two publications $[11,12]$ and below.

\subsubsection{Rutile}

The rutile structure (Fig. 1), adopted by $\mathrm{TiO}_{2}$ and also the isomorphous high pressure silica polymorph stishovite and a polymorph of $\mathrm{GeO}_{2}$ ) offers a salutory example. In this structure, parallel strings of edge-sharing $\left[\mathrm{TiO}_{6}\right]$ octahedra are interconnected through vertices shared across strings in $\{6,3\}$ connectivity. A vertex involved in a shared edge has one degree of freedom less. If involved in two independent edges, then some freedom would still remain; but if involved in two dependent edges (belonging to the same polytope), then there may or may not be freedom remaining. Confusion can arise in the calculation of $Y$ in eqn (1) if a global enumeration of vertices involved in shared edges is performed. If every vertex were involved in at least one shared edge, then $Y=1$; but vertices involved in two or more shared edges may be more constrained, and there needs to be some way to differentiate these degrees of constraint. From the more local point of view of a single polytope, what is important is whether one of its vertices is involved in an edge share with that polytope and another polytope.

This distinction is important for rutile, in which the oxygen atoms at every vertex are common to three octahedra $(C=3)$ and every oxygen is involved in a shared edge 
Table 1. Coordination, connectivity, topological freedom and amorphizability for some ceramic compounds with single and multiple polytopes and sharing modes

\begin{tabular}{|c|c|c|c|c|c|}
\hline Structure & Polyhedra : sharing & $\{V, C\}$ & $f$ & $\begin{array}{l}\text { Amorphization } \\
\text { dose }(\mathrm{eV} / \text { atom })^{\mathrm{a}}\end{array}$ & Ref. \\
\hline $\mathrm{MgO}$ & Octahedra : edges & $\{6,6\}$ & -10 & 5000 & [57] \\
\hline $\mathrm{UO}_{2}$ & Cubes : edges & $\{8,4\}$ & -7 & $>3000$ & [58] \\
\hline $\mathrm{MgAl}_{2} \mathrm{O}_{4}$ & $\begin{array}{l}\text { Octahedra : edges; } \\
\text { tetrahedra : } \text { corners }^{\mathrm{c}}\end{array}$ & $\begin{array}{l}\{4,4\} \\
\{6,4\}\end{array}$ & -5 & $400^{b}$ & [59] \\
\hline$\alpha-\mathrm{Al}_{2} \mathrm{O}_{3}$ & Octahedra : faces, edges \{ & $6,4\}$ & -5 & 380 & [60] \\
\hline $\mathrm{CaTiO}_{3}$ & $\begin{array}{l}\text { Octahedra : corners }{ }^{\mathrm{c}} \\
\text { truncated cubes }: \text { faces }\end{array}$ & $\begin{array}{l}\{6,2\}^{\mathrm{c}} \\
\{12,4\}\end{array}$ & $<-1$ & 89 & [61] \\
\hline $\begin{array}{l}\mathrm{TiO}_{2} \\
\text { (rutile) }\end{array}$ & Octahedra : edges, corners & $\{6,3\}$ & -3.7 & 75 & [62] \\
\hline $\mathrm{Mg}_{2} \mathrm{SiO}_{4}$ & $\begin{array}{l}\text { Octahedra : edges; } \\
\text { tetrahedra : edges, corners }{ }^{c}\end{array}$ & $\begin{array}{l}\{6,4\} \\
\{4,4\}^{\mathrm{c}}\end{array}$ & -3.4 & 79 & [35] \\
\hline $\begin{array}{l}\mathrm{Al}_{2} \mathrm{SiO}_{5} \\
\text { (sillimanite) }\end{array}$ & $\begin{array}{l}\text { Octahedra : edges; } \\
\text { tetrahedra : corners }{ }^{\mathrm{c}}\end{array}$ & $\begin{array}{l}\{6,3\} \\
\{4,3\}^{\mathrm{C}}\end{array}$ & -2.2 & 40 & [35] \\
\hline $\mathrm{SiC}$ & Tetrahedra : corners & $\{4,4\}$ & -3 & 13 & [63] \\
\hline $\mathrm{Si}_{3} \mathrm{~N}_{4}$ & Tetrahedra : corners & $\{4,3\}$ & -1.5 & $57^{d}$ & [64] \\
\hline $\mathrm{ReO}_{3}$ & Octahedra : corners & $\{6,2\}$ & -1 & $35^{e}$ & {$[65]$} \\
\hline $\mathrm{SiC}$ & Tetrahedra : corners & $\{4,2\}$ & $<0$ & 13 & [63] \\
\hline$C$ (graphite) & Triangles : corners & $\{3,2\}$ & $<0$ & 15 & [66] \\
\hline Si & Tetrahedra : corners & $\{4,2\}$ & $<0$ & 11 & {$[67]$} \\
\hline $\mathrm{CaSiO}_{3}$ & $\begin{array}{l}\text { Octahedra : edges; } \\
\text { tetrahedra : corners }{ }^{\mathrm{c}}\end{array}$ & $\begin{array}{l}\{6,3\} \\
\{4,2\}^{\mathrm{c}}\end{array}$ & $<0$ & 11 & [68] \\
\hline $\mathrm{Be}_{2} \mathrm{SiO}_{4}$ & Tetrahedra : corners & $\{4,2\}$ & 0 & 11 & [68] \\
\hline $\mathrm{AlPO}_{4}$ & Tetrahedra : corners & $\{4,2\}$ & 0 & 10 & [69] \\
\hline $\mathrm{SiO}_{2}$ & Tetrahedra : corners & $\{4,2\}$ & 0 & 7 & [68] \\
\hline $\mathrm{P}_{2} \mathrm{O}_{5}$ & Tetrahedra : corners & $\{4,1.6\}$ & +0.6 & $0.5^{f}$ & [70] \\
\hline $\mathrm{B}_{2} \mathrm{O}_{3}$ & Triangles : corners & $\{3,2\}$ & +1 & g & \\
\hline
\end{tabular}

\footnotetext{
${ }^{a}$ Low-temperature values of critical energy density required for amorphization well below critical amorphization temperature. ${ }^{b}$ Cation sublattice. ${ }^{c}$ Weak-link connectivity. ${ }^{d}$ Highly sensitive to implanted

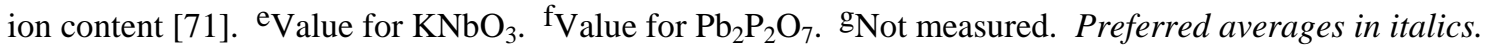




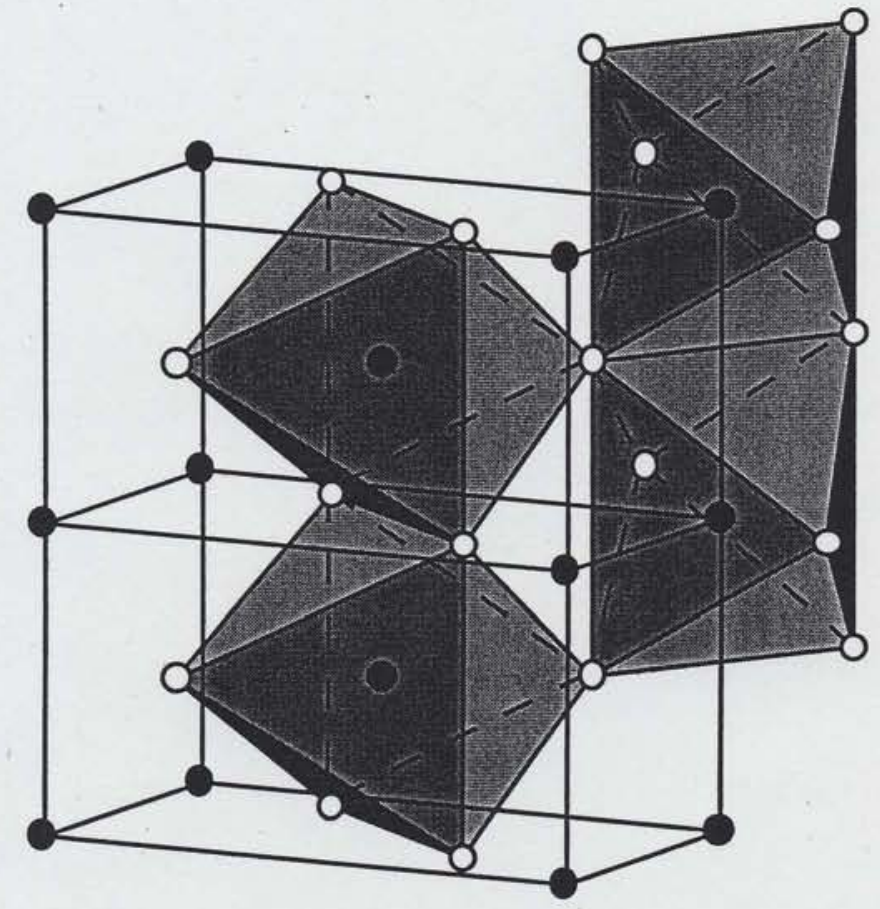

Ti OO

[ $\left.\mathrm{TiO}_{6}\right],\{6,3\}, \mathrm{Y}=0.66, \mathrm{f}=-3.7$

Fig. 1 Rutile structure of $\mathrm{TiO}_{2}$, showing edge and corner sharing of $\left[\mathrm{TiO}_{6}\right]$ octahedra. 
linking two octahedra. However, a given octahedron has two shared edges, with only 4 of its 6 oxygens involved in those shared edges, hence $Y=4 / 6$, not 1 as defined by Gupta [56]. The result of this edge sharing is a large reduction in topological freedom (to $f_{a v g}=$ -3.7), borne out by the relative difficulty of amorphization of $\mathrm{TiO}_{2}$ rutile (recently measured [62] at $75 \mathrm{eV} /$ atom, $0.75 \mathrm{dpa}$ ).

\subsubsection{Corundum}

The corundum structure of alumina $\left(\alpha-\mathrm{Al}_{2} \mathrm{O}_{3}\right)$ offers a similarly instructive example for simultaneous edge and face sharing. The face sharing is very inhomogeneously distributed, confined to isolate pairs of octahedra that are only edgeshared with other octahedra. The arrangement of six such pairs is defined in the depiction of successive basal layers of octahedra in Fig. 2. Every octahedron is paired with another in just such a way, face-sharing one of its 8 triangular faces with a second tetrahedron in the adjacent basal layer; of its twelve edges, 3 are consumed in the shared face, 3 are shared with surrounding octahedra, and 6 remain unshared. Eqn (1) thus becomes $f=-5-Y-Z$. All six oxygen vertices are involved in the shared edges, but half of these are in the shared face, so $Y=1 / 2$ and $Z=1 / 2$. In this way, eqn (1) calculates $f_{\text {avg }}$ $=-6$, but this is in fact an overestimate of the constraint. Because of their isolation, the shared faces contribute nothing to the network rigidity; instead they define a "superpolytope" (also illustrated in Fig. 2) which conveniently offers a neater and wholly unambiguous way to enumerate the rigidity, The super-polytope comprises two facesharing octahedra with 9 vertices (an $\left[\mathrm{Al}_{2} \mathrm{O}_{9}\right]$ unit), which is connected to 3 other such polytopes by a single edge-sharing mode in which all vertices participate $(Y=1)$. This arrangement yields $f_{s p}=-5$.

\subsubsection{Spinel}

Oxide spinels (e.g. $\mathrm{MgAl}_{2} \mathrm{O}_{4}$ ) represent a particularly resilient class of structure whose connectivity is well distributed, clearly the secret to its notable [59] resistance to amorphization. In this structure, cations occupy tetrahedral and octahedral sites in a cubic close packed (сср) arrangement of oxygen anions. There are twice as many octahedra (which are in $\{6,4\}$ connectivity) as tetrahedra (in $\{4,4\}$ connectivity): in normal spinel, the $\mathrm{Mg}^{2+}$ cations occupy the designated tetrahedral sites and $\mathrm{Al}^{3+}$ cations the designated octahedral sites, though redistribution is possible, for example during irradiation. All $\mathrm{O}^{2-}$ anions are equivalent, each being part of 3 octahedra and 1 tetrahedron and also participating in 3 shared edges. Half the octahedron edges are shared, but every vertex in an octahedron participates in that edge sharing (so $Y_{O}=1$ ). The tetrahedra share only vertices, and only with the octahedra, and each vertex with respect to a tetrahedron is involved only in vertex sharing of that tetrahedron $(Y=0)$. Hence, for the octahedra $f_{o}=-6$ and for tetrahedra $f_{t}=-3$, yielding the globally weighted average $f_{\text {avg }}=-5$. Alternatively, applying eqn (1) to vertices in the octahedral and tetrahedral polytopes, $f_{\text {avg }}=3-6-1.5=-4.5$, less the constraint $\left(+1\right.$ if $\left.Y_{O}=1\right)$ provided by the octahedral edge sharing, yielding $f_{a v g}=-5.5$. The globally weighted value of $Y$ in

this case is certainly not as large as unity, however. We believe that averaging the 

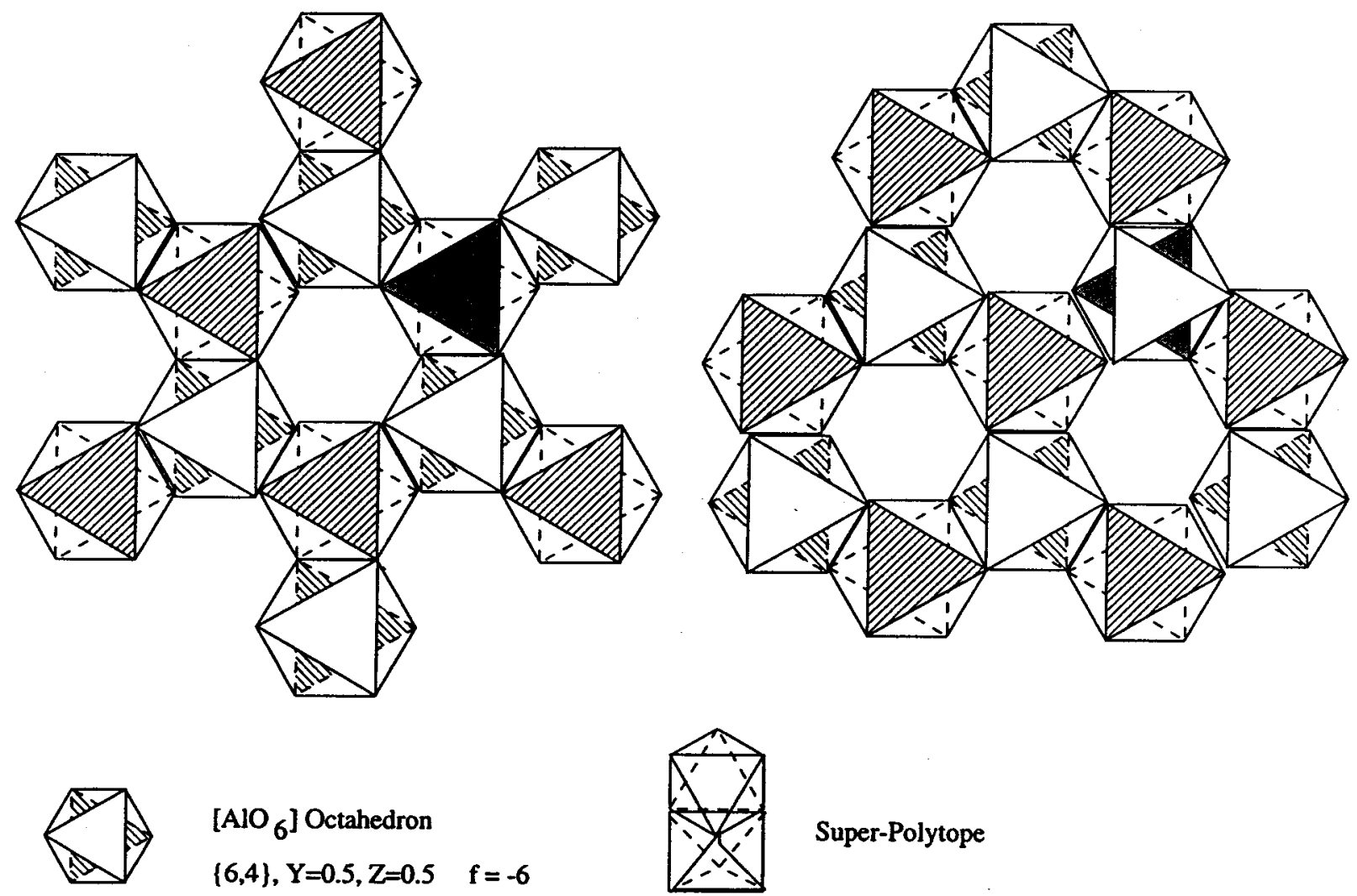

Super-Polytope

Fig. 2 Successive (0001) layers of corundum structure of $\alpha-\mathrm{Al}_{2} \mathrm{O}_{3}$, illustrating edge sharing of $\left[\mathrm{AlO}_{6}\right]$ octahedra and face sharing (shaded) of octahedra in an $\left[\mathrm{Al}_{2} \mathrm{O}_{9}\right]$ super-polytope illustrated. 


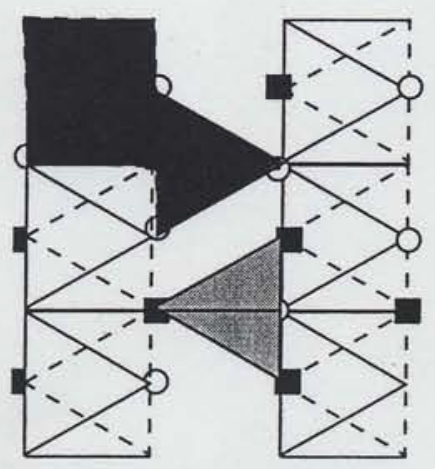

level 0

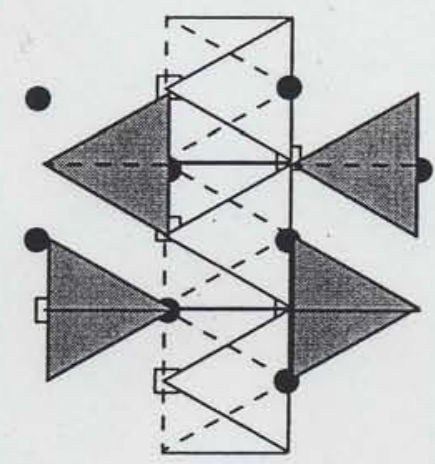

level 2

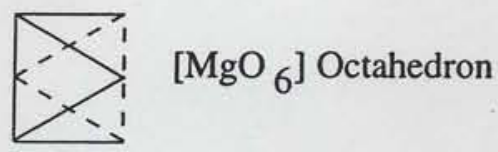

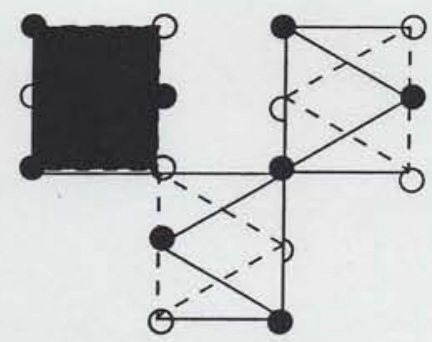

level 1

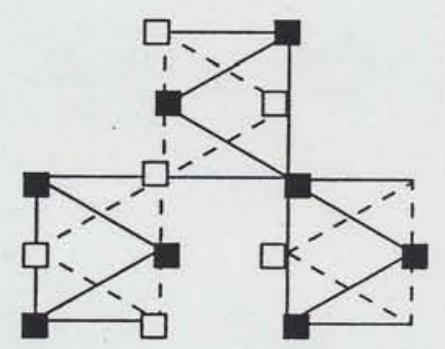

level $3=-1$

$\left[\mathrm{SiO}_{4}\right]$ Tetrahedron

Fig. 3 Four successive layers in the olivine structure of $\mathrm{Mg}_{2} \mathrm{SiO}_{4}$, showing chains of edgesharing $\left[\mathrm{MgO}_{6}\right]$ octahedra and linking $\left[\mathrm{SiO}_{4}\right]$ tetrahedra. The constituents of an $\left[\mathrm{Mg}_{2} \mathrm{SiO}_{11}\right]$ rigid super-polytope are shaded. 
separate polytopic freedoms is the better method of enumeration and that for spinel $f=-5$ is the correct topological freedom.

\subsubsection{Olivine}

Forsterite $\left(\mathrm{Mg}_{2} \mathrm{SiO}_{4}\right)$, which has the olivine structure, is a hexagonal analogue to the cubic spinels. Though it is a compound of identical stoichiometry to spinel, it is far less resistant to irradiation-induced amorphization [35]. The $\mathrm{Mg}^{2+}$ cations form $\left[\mathrm{MgO}_{6}\right]$ octahedra arranged in edge-sharing chains, while the $\mathrm{Si}$ atoms form isolated $\left[\mathrm{SiO}_{4}\right]$ tetrahedra that share 3 of their 6 edges and one corner with the chains of $\left[\mathrm{MgO}_{6}\right]$ octahedra (Fig. 3). As in spinel, the octahedra share half their edges, and their oxygen vertices are connected to 3 octahedra and one tetrahedron. Unlike spinel, there are two different kinds of vertices. The first (comprising 75\%) are part of 3 octahedra and one tetrahedon, and all participate in edge sharing $\left(Y_{1}=1\right)$, yielding a contribution $f_{1}=-5.5$, as in spinel. The others (25\%) do not participate in edge sharing $\left(Y_{2}=0\right)$, yielding the contribution $f_{2}=-4.5$. The species-weighted average is $f_{\text {avg }}=-5.25$. Alternatively, forsterite can be considered to be comprise two sorts of $\left[\mathrm{MgO}_{6}\right]$ octahedra, distinguishable by their environments $\left(V=6, C=4, Y_{01}=1, f_{o 1}=-5.75\right.$ and $C=4, Y_{o 2}=$ $\left.0.5, f_{2}=-5.5\right)$, and $\left[\mathrm{SiO}_{4}\right]$ tetrahedra $\left(V=4, \mathrm{C}=4, Y_{t}=0.75, f_{t}=-3.75\right)$, for a weighted average of $f_{\text {avg }}=-5.2$, very close to the vertex-based estimate.

Both these averages, while adequate for spinel, do not correctly represent the susceptibility of the rigidity to removal of oxygens at the second kind of vertex. The super-polytope approach, discussed for alumina, can be advantageously applied to the olivine case in this instance to obviate the difficulty. A rigid $\left[\mathrm{Mg}_{2} \mathrm{SiO}_{11}\right]$ super-polytope can be defined (Fig. 3) which comprises two edge-sharing octahedra and one tetrahedron and has 11 vertices. Eight of these vertices are shared between three super-polytopes, 2 between two super-polytopes and one is non-bridging. Therefore, $C_{a v g}=11(8 / 3+2 / 2+$ $1)=2.36$. Also, there are 6 vertices involved in face sharing of the super-polytopes, so that $Z=6 / 11$. The resulting freedom, $f_{s p}=-3.4$, is much smaller than the global average and more accurately reflects the weak link provided by the vertices that do not participate in edge sharing.

\subsection{Polytope Choice in Elemental Networks}

The choice of polytope with which to represent a structure is important for two reasons: It is necessary to represent correctly the actual constraints, and a given choice may lead to an easier enumeration of the available structural freedoms. For predictably coordinated compounds, the coordination unit is likely to persist intact and undistorted in any reorganization of the structure, and the choice of polytope is obvious. For monatomic solids, like $\mathrm{C}$ or $\mathrm{Si}$, the choice is less obvious.

\subsubsection{Graphite}

In graphite, $\mathrm{C}$ atoms are 3-connected through $\mathrm{sp}^{2}$ hybridized bonds in planar hexagonal nets (Fig. 4) only loosely bound to neighboring sheets. For the choice of a 
[CC] rod polytope, graphite can be represented by $\{2,3\}(\delta=1, \mathrm{~d}=2)$, in which representation $f=+1$. Given the experimental amorphizability (15 eV/atom [66], Table 1) for graphite-close to that of $\mathrm{SiO}_{2}$ for which $f=0$ - this representation clearly underestimates the structural constraints. Under-representation occurs because the trigonal directionality of the $\mathrm{C}-\mathrm{C}$ bonds has been ignored: the bonding geometry introduces angular constraints that must be accounted for; these are largely steric and weaker for metallic bonding, as indicated, but are strong constraints for highly directional covalently bonded carbon.

A more appropriate choices of polytope is an equilateral triangle for graphite. In Fig. 4, a $\left[\mathrm{CC}_{3}\right]$ triangular polytope $(\delta=2)$ is seen to be $\{3,3\}$-connected, resulting in $f=$ -1 . The difficulty with this representation, which now overestimates the experimentallydeduced constraints, is that it requires two distinguishable species of carbon atom, one at the polytope center, the other at the vertices, which is unphysical. A still better representation is the $\left[\mathrm{C}_{-3}\right]$ shaded triangles centered on each $\mathrm{C}$ atom with vertices at the bond midpoints; these are $\{3,2\}$-connected, with $f=0$. In this representation, the Cvertex-C angle is required to be $180^{\circ}$ (corresponding to the straight [CC] rod), whereas in the $\{3,2\}$ arrangement of triangles depicted in Fig. 4 this angle is free; hence, there is less freedom in the graphite structure than in the $\{3,2\}$ representation, and $f$ is somewhat $<0$, as suggested by the $15 \mathrm{eV} /$ atom amorphization dose. Flexibility in this C-vertex-C angle corresponds to a distortion of the $120^{\circ} \mathrm{sp}^{2}$ bonds, which means that amorphization of graphite must be accompanied by bond-angle changes.

\subsubsection{Silicon and Diamond}

A similar situation obtains for the diamond structure, into which both carbon and silicon can assemble, with 4-coordination resulting from hybridized $\mathrm{sp}^{3}$ tetrahedral bonds (Fig. 5). Representation of the tetrahedral structure by [SiSi] (or [CC]) rods (for which representation $\{C, V\}=\{2,4\}, \delta=3, d=3$, and $f=+1$ ) leads to an under-representation of the tetrahedral bond constraint. Two alternative tetrahedron arrangements are possible. In the first of the these, $\left[\mathrm{SiSi}_{4}\right]$ (or $\left[\mathrm{CC}_{4}\right]$ ) tetrahedra are $\{4,4\}$-connected, but the representation clearly overstates $(f=-3)$ the rigidity of the network for the same reason that $\left[\mathrm{CC}_{3}\right]$ triangles fail to represent graphite: the indistinguishability of the $\mathrm{Si}$ (or $\mathrm{C}$ ) atoms. The alternative is $[\mathrm{Si}-4]$ (or $[\mathrm{C}-4]$ ) tetrahedra with vertices at the bond midpoints which are $\{4,2\}$-connected. As in the analogous representation of graphite, the Si-vertexSi (or C-vertex-C) angle is constrained to be $180^{\circ}$ if (in this case) the $109.5^{\circ}$ tetrahedral bond angles are to be maintained; to the extent that the tetrahedral angle can be altered, the structure approaches the topological freedom $(f=0)$ of the $\{4,2\}$ network of $\mathrm{SiO}_{2}$. The experimental amorphizability of Si (11 eV/atom [67], Table 1) suggests a value close to $f=0$. Hence, amorphization of silicon must also be accompanied by bond-angle changes.

\subsection{Chemical Disorder}




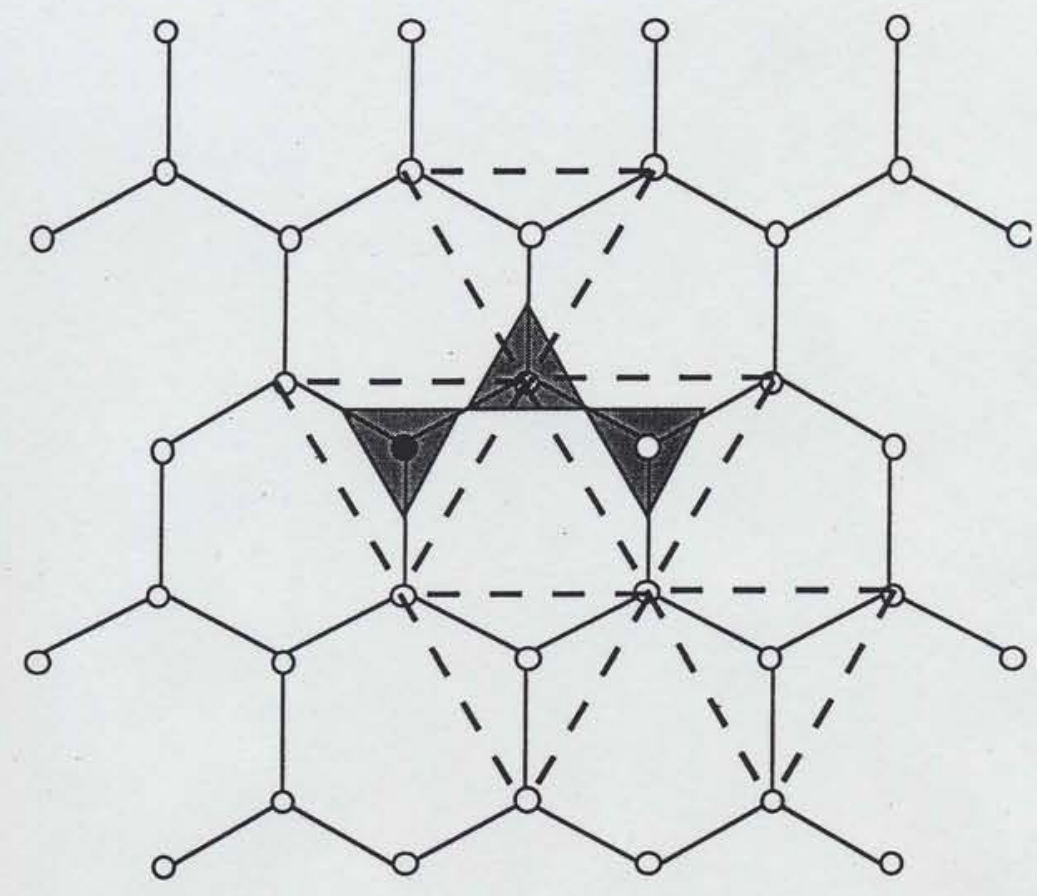

$\longrightarrow$ Rods: $\{2,3\}, \delta=1, f=+0.5$

Triangles: $\{3,3\}, \delta=2, f=-1$

$\nabla$ Triangles (to bond midpoint): $\{3,2\}, \delta=2, \mathrm{f}=0$

Fig. 4 Hexagonal two-dimensional network of graphite (0001) sheet, with three connectivity repreentations, using $[\mathrm{CC}]$ rods, $\left[\mathrm{CC}_{3}\right]$ coordination triangles, and $\left[\mathrm{C}_{3}\right]$ triangles with vertices at $\mathrm{C}-\mathrm{C}$ bond midpoints. 

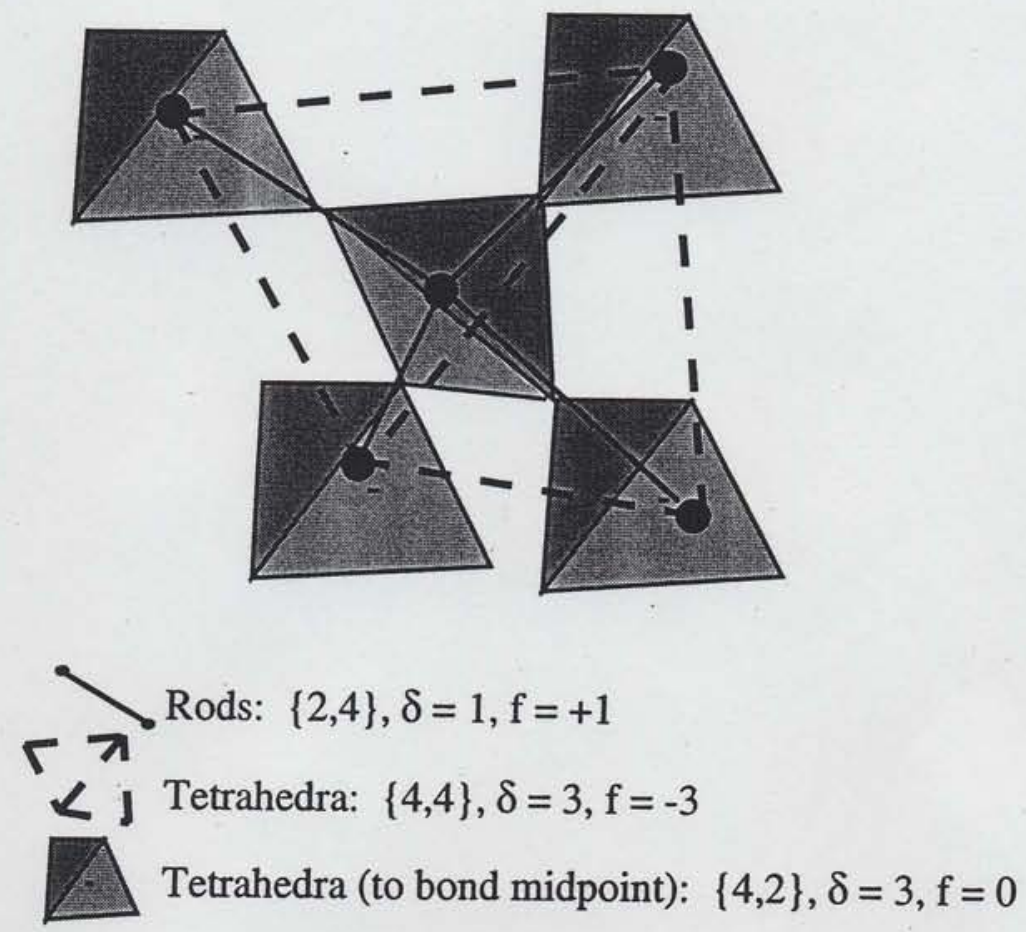

Fig. 5 Diamond structure of silicon, with three connectivity representations, using [SiSi] rods, $\left[\mathrm{SiSi}_{4}\right]$ coordination tetrahedra, and $\left[\mathrm{Si}_{4}\right]$ tetrahedra with vertices at the $\mathrm{Si}-\mathrm{Si}$ bond midpoints. 
The coordination polytope representation of structures for the purposes of amorphizability presupposes that the polytope identities will not change during irradiation, that is, that the central atom or ion of the polytope will not change identity or coordination. This assumption is not always warranted when multiple sites are available for occupation, when the valence of a cation can be altered, or when atoms of different species are interchangeable with little energy cost.

\subsubsection{Spinel}

In normal $\mathrm{MgAl}_{2} \mathrm{O}_{4}$ spinel, for example, in which ideally the $\mathrm{Mg}^{2+}$ cations occupy one-eighth of the tetrahedral interstitial sites in a cubic close-packed (сср) oxygen sublattice and $\mathrm{Al}^{3+}$ half the octahedral sites, it is well known that partial inversion of the site occupations regularly occurs. During irradiation, it is likely that an even more random redistribution of the two cations among all available normally occupied sites (as well as those not normally occupied) may occur. Under ion irradiation, spinels appear to disorder in two stages [59,71,72]. In the first, the cation sublattice appears to disorder after about $400 \mathrm{eV} /$ atom ( $~ 4 \mathrm{dpa}$ ), leaving the oxygen sublattice intact; in the second, something approaching complete amorphization is observed at $3500 \mathrm{eV} /$ atom (35 dpa) at cryogenic temperature. The former value is in accord with the topological freedom $(f=-$ 5) calculated above for spinel. The latter represents the dose to amorphize a structure which is closer to that for the defect rocksalt structure that would obtain if the cations were distributed uniformly over 3/4 of the octahedral interstices of the cсp oxygen sublattice by irradiation-induced disordering.

\subsubsection{Silicon Carbide}

Silicon carbide, in either cubic $\beta$ form or any of its $\alpha$ polytypes, may be represented topologically as a $\{4,4\}$ structure comprising $\left[\mathrm{SiC}_{4}\right]$ (or alternatively $\left[\mathrm{CSi}_{4}\right]$ ) tetrahedra joined four at a vertex. The corresponding topological freedom value $(f=-3)$ suggests that $\mathrm{SiC}$ should be highly constrained and difficult to amorphize, a conclusion inconsistent with its surprising ease of amorphization (13 eV/atom [63]). Its ready amorphizability (below 298-500 K depending on irradiation type) can be explained by the ability of $\mathrm{SiC}$ to sustain chemical disorder in the form of $\mathrm{C}_{\mathrm{Si}}$ or $\mathrm{Si}_{\mathrm{C}}$ substitutions. These substitutions, with relatively small energy cost, lead to creation of formal [ $\left.\mathrm{SiSi}_{4}\right]$ and $\left[\mathrm{CC}_{4}\right]$ antisite defects and the whole range of partial $\left[\mathrm{Si}-\mathrm{SiC}_{3}\right],\left[\mathrm{Si}-\mathrm{Si}_{2} \mathrm{C}_{2}\right],\left[\mathrm{Si}-\mathrm{Si}_{3} \mathrm{C}\right]$, etc. defect configurations, which are discussed more fully in $\S 4$. Collisional disorder may thus generate an average diamond structure with random occupation of sites by $\mathrm{Si}$ and $\mathrm{C}$ atoms, leading to a topological freedom similar to that of silicon ( $f=0$, as argued above) with an average atom. The replacement-to-displacement ratio is higher for cascade displacements than for single atom displacements, so it can be expected that ion irradiation will be more efficient (per unit deposited displacement energy) than electron irradiation in inducing amorphization, which appears to be the case [73]. Recent MD simulations of collision cascades in SiC [74] show that a large number of partial antisite defects are formed in the resulting amorphous clusters, and indeed recent EXAFS and Raman experiments [75-77] in ion-amorphized $\mathrm{SiC}$ have detected substantial C-C coordination. Anti-site defects are likely to anneal thermally at modest temperatures in 
SiC, accounting for (or at least contributing to) the lower ( 290-340 K) observed critical temperature for amorphization by electron irradiation [20,78] compared to that $(\sim 420-498$ K) for ion-irradiation induced amorphization [63].

\subsection{Functional Correlation of Amorphizability and Toplogical Freedom}

The various rationalized values for topological freedom established in Table 1 indicate a strong correlation with measured amorphizabilities (at least in terms of collisional energy deposition per atom for ion irradiation). The correlation can be rendered into more quantitative form by plotting the logarithm of $|f-3|$ against the logarithm of the amorphization dose $D$ (Fig. 6). The result, which is surprisingly linear across the whole four orders of magnitude in critical amorphization dose, corresponds to the approximate functional relationship

$$
f=3-2.51 D^{0.187}
$$

The topological justification for choice of this form for eqn (2) is the recognition that irradiation successively destroys the constraints on each polytope vertex (which without constraints would ultimately end up with three degrees of freedom in three-dimensional structures). From a comparison of eqns (1) with (2), it is evident that the term $2.51 D^{0.187}$ in eqn (2) represents the destruction of the vertex constraints by irradiation sufficient to render a structure topologically floppy. The reason why a structure like those of silica, with $f=0$ already, or a phosphate with $f>0$, should require additional input of bondrupture energy is that introduction of topological disorder requires rebonding; mere floppiness (e.g. presence of soft rigid-unit modes [79]) is not sufficient for amorphization. The particular power law in eqn (2) will depend on the kinetic model [80] adopted for the amorphization process and its back reactions, which must in any case be similar for the range of structures represented.

Fig. 6 can be used to predict the effective topological freedom in structures (e.g. perovskites) for which the contribution of the several structural elements is unclear and can increase appreciation of what features confer structural stability. For example, eqn (2) predicts $f=-2.8$ for $\mathrm{CaTiO}_{3}$, a structure comprised of vertex-sharing $\left[\mathrm{TiO}_{6}\right]$ with the resulting cuboctohedral interstices stuffed with large $\mathrm{Ca}^{2+}$ cations. This result suggests that the effect of the highly-coordinated $\mathrm{Ca}^{2+}$ ion and its face-sharing $\left[\mathrm{CaO}_{12}\right]$ cuboctahedral polytopes on structural rigidity of a corner-sharing network of octahedra (for which $f=-1$, as in the $\mathrm{ReO}_{3}$ structure) is certainly not negligible, but by no means overriding either.

The topological approach is, of course, purely geometrical and (apart from the considerations of polytope preference and directional bonding already described) does not take into account further influences of chemistry or atomic species. These influences are clearly important: for example, $\mathrm{CaTiO}_{3}$ amorphizes after 89 eV/atom (0.89 dpa [65]), while another isostructural perovskite $\mathrm{KNbO}_{3}(0.35 \mathrm{dpa}$, assuming similar displacement energies [80]) is more easily amorphizable. Amorphization involves a complicated competition between topological capacity to sustain disorder and the energetic motivation to recrystallize (often epitaxially on adjacent undamaged crystal). An alternative semi- 


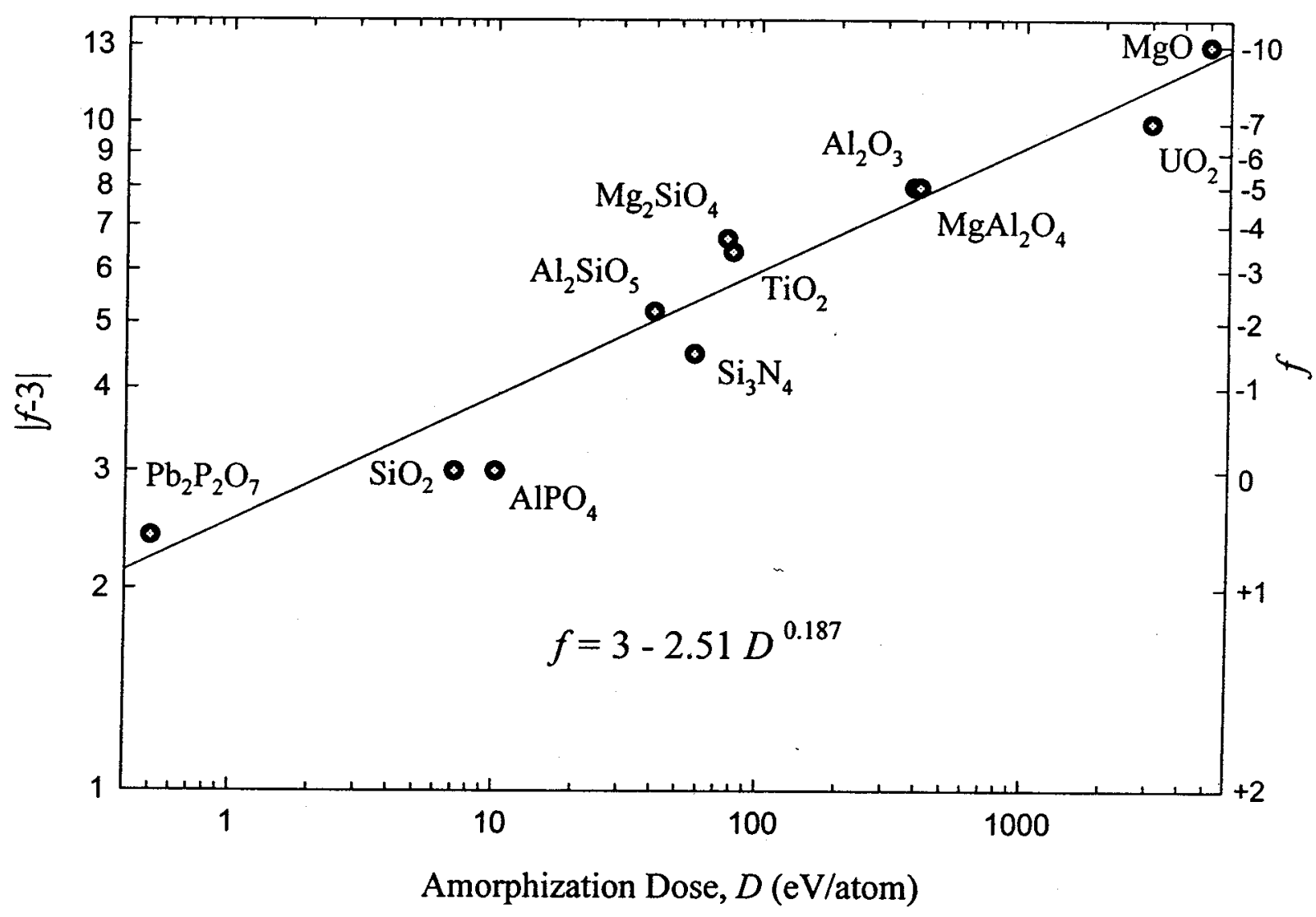

Fig. 6 Correlation of topological freedom $f$ with amorphization dose $D$ in $\log \log$ plot of $|f-3|$ against $D$. The line is a non-linear least squares fit to the experimental amorphization doses given in $\mathrm{eV} /$ atom. 
empirical approach [36] has considered the chemistry of amorphization more explicitly in the form of a semi-empirical parameter, incorporating rigidity and measured physical parameters. In particular, this approach invokes the correlation of crystallization with melt viscosity to predict amorphizability within a more restricted suite of compounds in the $\mathrm{MgO}-\mathrm{Al}_{2} \mathrm{O}_{3}-\mathrm{SiO}_{2}$ ternary system [35].

\section{MOLECULAR DYNAMICS MODELING OF AMORPHIZED SILICAS}

Detailed information about the structure and structural changes in crystalline solids can be derived (on average) from X-ray, neutron or electron diffraction information or directly from high-resolution electron microscopy. Equivalent information is not available for amorphous or amorphized solids. Diffraction measurements yield structurally average diffracted intensities, from which it is possible to obtain information only about directionally and species-averaged pair correlations, expressed in the form of radial density functions (RDFs) or species-averaged total correlation functions $T(r)$, by Fourier transformation [81]. Such diffraction-based data provide accurate information about short-range correlation distances and-somewhat less reliably-about coordination number within the first atom correlation shell (e.g. the $\left[\mathrm{SiO}_{4}\right]$ tetrahedron in all tetrahedral silicates), both of which are likely to change little from one silica structure to the next. Apart from small differences in Si coordination, it is only at second- or mostly at third-neighbor tetrahedra that there are any significant topological distinctions in tetrahedral silica structures, and $T(r)$ is notoriously insensitive to differences in intermediate order in this range [82].

Structural modeling of topologically disordered silicas represents a productive alternative prospect from which to explore the structural bounds imposed by topological possibilities. Two different approaches are useful. In the first, topological rules or assembly algorithms are used to erect structural models with desired topologies. Since such topologies are based on local connectivities and not energetics, with no need to specify discrete atom positions, a second sequential method must be used to ensure adequate global connectivity and generate a set of energy-minimized atom coordinates to which to compare experimental information derived from diffraction data. One method to ensure maximal connectivity (but not optimal coordinates) can be based on graph theory. The most powerful such method for both appears to be molecular dynamics, used not in the usual fashion to evolve a structure de novo but instead to refine a rough set of coordinates established by prior topological considerations. These approaches are discussed for silica in this section and for $\mathrm{SiC}$ in the next.

\subsection{Assembly and Reassembly of Networks}

Most forms of silica, crystalline or amorphous, comprise vertex-sharing network arrangements of $\left[\mathrm{SiO}_{4}\right]$ tetrahedral short-range-ordered units. An efficient way to envisage possibilities for the tetrahedral network arrangements is based on their topologies. Silica networks are representable as graphs, for which the intermediate (medium) range order at any tetrahedron can be characterized by the set of undecomposable closed circuits (primitive rings) of the network passing through that 
tetrahedron $[8,45,83-85]$. The collection of tetrahedra belonging to the set of primitive rings through a given tetrahedron we have called the local cluster of that tetrahedron and serves as a unit description of structure, based on topology, alternative to the crystallographic unit cell which is based on symmetries. Two examples of local clusters, for the quartz and cristobalite crystalline polymorphs of silica, are illustrated in Fig. 7. Unlike crystallographic representations, this characterization is not limited to crystalline networks and can be equally applied to amorphous network arrangements $[83,84]$.

In order to erect models of crystalline tetrahedral networks, generative algorithms can be devised based on local connectivities. A rules-based self-assembly procedure established for the purpose, utilizing local assembly rules to generate all the compact crystalline polymorphs of $\mathrm{SiO}_{2}$, has been discussed in the research proposal for the previous grant period, outlined in detail in a comprehensive journal treatment [85], and reviewed at length in a recent book chapter [45]. These polymorphs are seen to be characterizable by a characteristic average ring size and local cluster size which both scale monotonically with polymorph density (Table 2).

\subsubsection{Amorphous Constructions}

Silicas are readily amorphizable because-as seen in §2—sufficient topological freedom is avalable for the networks to be only marginally rigid. Topological disorder results because multiple reconstructive stochastic paths are available during reassembly into stable solid forms from the liquid or radiation-disordered precursor states. In one approach to modeling such reconstructions [85], local crystalline assembly rules are modified to introduce frustration into the assembly, resulting in amorphous silica structures. Reconnection under such circumstances can be crudely maximized by attaching Hookeian springs between tetrahedron vertices which do not quite match up and minimizing the elastic energy in all such springs globally at each assembly step. Amorphous silicas with quite distinct topologies can be thus generated, such as those based on cristobalite-like or quartz-like assembly algorithms (Table 2). The observed correlation of ring size and local cluster size with density, together with the model results, suggest that vitreous silica is dominated by a cristobalite-like 6-ring average, while metamict silica likely involves some larger ring structures to account for its higher density.

Radiation-induced direct-impact amorphization in collision cascades provides a second legitimate venue for topological modeling, by using local rules reassembly following a massive disordering event that leaves reconstituted tetrahedra positionally and rotationally randomized. Even if amorphization occurs less directly, through accumulation of individual defects, critical accumulation of disorder could trigger a similar reconstruction. For this approach [86], initial structures were generated using local rules assembly algorithms. Crystalline models comprising 2000 tetrahedra of $\alpha$ cristobalite, $\alpha$-quartz and ideal quartz were erected; connections between the central 400 tetrahedra were severed and the boundary adjoining this volume was expanded. Tetrahedra within the central volume were randomly rotated to simulate uncorrelated cascade displacements, then allowed to reform connections to each other and to the expanded boundary according to a chosen set of rules. A non-crystalline ("amorphous silica”) model comprising 1500 tetrahedra was analogously erected using modified 


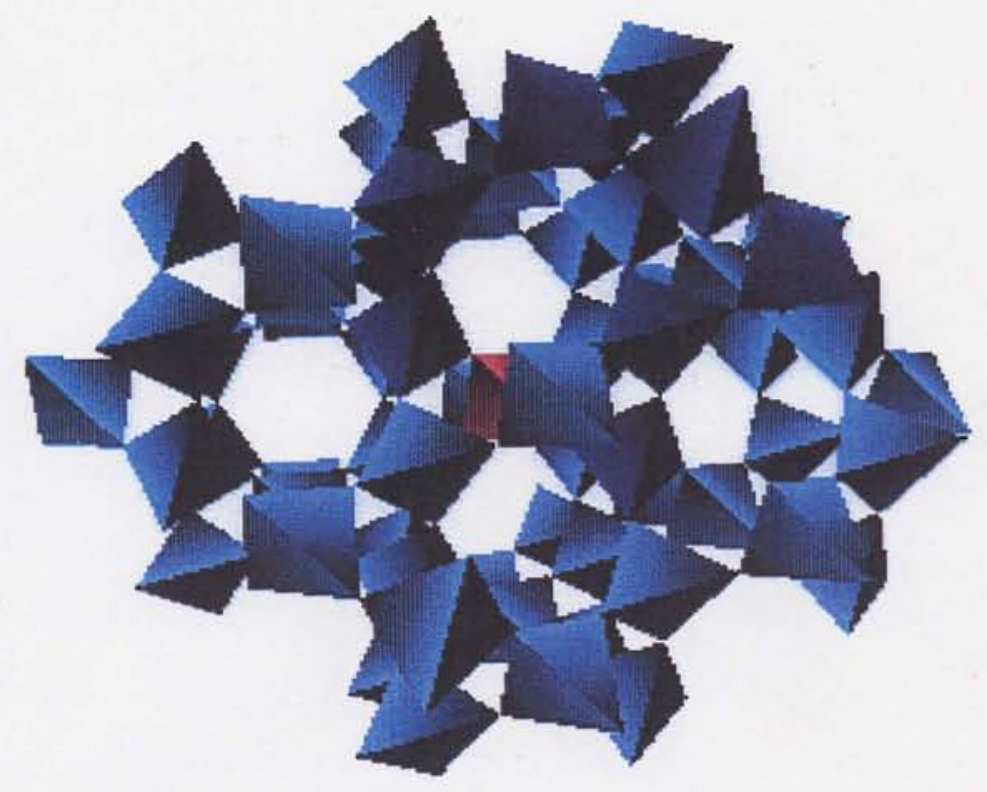

(a) 63 tetrahedra

6 6-rings

40 8-rings

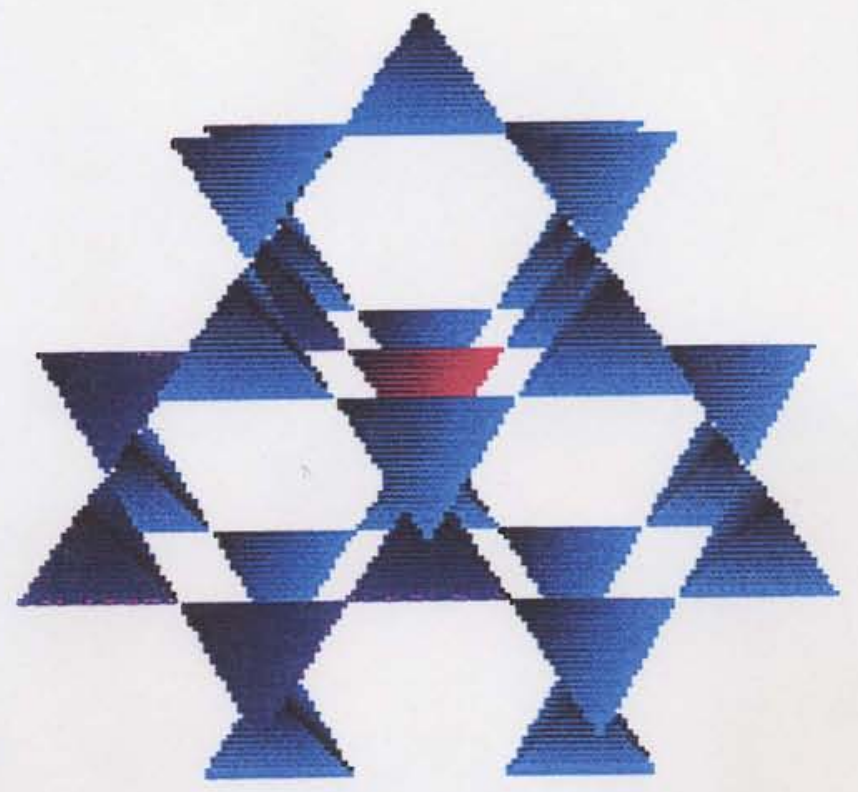

(b) 29 tetrahedra

12 6-rings

Fig. 7 Local clusters for the a) quartz and b) cristobalite crystalline polymorphs of silica [85]. 
Table 2. Geometrical and topological parameters for silica models. Correlations of density with average ring and local cluster size are highlighted.

\begin{tabular}{|c|c|c|c|c|c|}
\hline Polymorph/ & $\begin{array}{c}\text { Avg. Si-O-Si } \\
\text { Angle } \theta^{\circ}\end{array}$ & $\begin{array}{c}\text { Avg. } \\
\text { Ring Size }\end{array}$ & $\begin{array}{c}\text { Avg. Local } \\
\text { Cluster Size }\end{array}$ & $\begin{array}{c}\text { Model Density, } \\
\times \mathbf{1 0}^{3} \mathbf{~ k g} / \mathbf{m}^{3}\end{array}$ & $\begin{array}{c}\text { Actual Density, } \\
\times \mathbf{1 0}^{3}{\mathbf{~ k g} / \mathbf{m}^{3}}^{3}\end{array}$ \\
\hline \hline HP-Tridymite & 149.5 & 6 & 27 & 2.22 & 2.18 \\
\hline$\beta$-Cristobalite & 148 & 6 & 29 & 2.17 & 2.21 \\
\hline$\alpha$-Cristobalite & 145.3 & 6 & 29 & 2.33 & 2.33 \\
\hline Keatite & 154.1 & 6.9 & 39 & 2.61 & 2.50 \\
\hline$\alpha$-Moganite & 138.5 & 7.4 & 41 & 2.72 & 2.62 \\
\hline$\alpha-Q u a r t z$ & 143.6 & 7.7 & 63 & 2.77 & 2.65 \\
\hline Coesite & 150.8 & 10.0 & 78 & 2.82 & 3.01 \\
\hline
\end{tabular}

\begin{tabular}{|c|c|c|c|c|c|}
\hline Vitreous Silica & 145 & $\sim 6 ?$ & - & - & 2.21 \\
\hline Metamict Silica & 134 & $>6 ?$ & & & 2.26 \\
\hline Cristobalite-like a-SiO 2 & 145.9 & 6.6 & 24.0 & 2.29 & - \\
\hline Quartz-like a-SiO 2 & 153.7 & 7.4 & 56.5 & 2.48 & - \\
\hline Cristobalite Cascade & 139.9 & 7.0 & 31.9 & 2.44 & \\
\hline
\end{tabular}


cristobalite assembly rules ( $85^{\circ}$ assembly rotations in place of $90^{\circ}$ in order to generate frustration) and the central 300 tetrahedra similarly disordered and reconnected. The tetrahedra were assumed for the purposes of the modeling to stay intact; they thus comprise the short-range units appearing in the initial rebonding phase of the cascade when $\left[\mathrm{SiO}_{4}\right]$ units reform. Both interior and boundary were lastly settled using the Hookeian-spring optimization scheme described above. Distortions in the tetrahedra were allowed to develop in response to their reconnections, as evidenced by remanent springs of non-zero length.

\subsubsection{Reassembly}

Two reassembly schemes were applied. In the first, reassembly was prescribed using a set of local rules, in each case based on a higher temperature polymorphic reconstruction, in order to reflect the high effective temperature within the cascade: $\alpha$ cristobalite was reconnected with $\beta$-cristobalite rules, $\alpha$-quartz with $\beta$-quartz rules, ideal quartz with $\alpha$-cristobalite rules, etc. Disordered amorphous silica was reconnected with the same rules as the initial assembly. The reassembly rules algorithms essentially emphasize the rotational relationships between tetrahedra. The resultant reassemblies end up substantially, but not fully, connected and have very different topologies [86], as evidenced by evaluation of their local cluster distributions (Table 2). Their remanent underconnection is addressable by subsequent refinement by molecular dynamics approaches discussed below.

A second reconnection scheme aims to maximize reassembly connectivity [84]. In this approach, based on graph theory, tetrahedra are disconnected and randomly rotated as before. Their vertices form a graph, edges of which are potential connections between vertices which are to be minimized in length in ensemble. A disjoint collection of edges is sought which is as numerous as possible; vertex separations up to 1.75-2.5 times an Si-O distance are included as edges of the graph so as to avoid too sparse a graph, and a minimal inter-tetrahedral angle $>90^{\circ}$ is required to preclude edge-sharing of otherwise vertex-sharing tetrahedra. The graph edges are next assigned weight according to an inverse weighting term, and an optimal match of vertices (the global maximal match) is found by maximizing the total graph weight. The matching algorithm used is based on a search for augmenting paths [87] (an augmenting path is one for which every second edge in the path is part of the match) and favors connectivity over angular relationships. Hookeian springs are added between matched vertices, and the total elastic energy in them in minimized as before. The global matches come out amorphous but fully connected, though with topologies (Table 2) rather closer to their originating polymorphic forms. This reconstruction algorithm may more closely simulate the global mechanistics of glass formation from a melt.

\subsection{Molecular Dynamics Refinement of Simulated Pseudo-Cascades}

Topological modeling as described above provides useful information about what arrangements of amorphous and metamict network structures are possible and tools for characterizing those arrangements. Its chief drawback is that atomic positions, while consistent with the topologies imposed or resulting, are not optimized with respect to any 
realistic interatomic potential (at best, crude Hookeian springs) and hence cannot be usefully compared to positions derived from experimental evidence, for example from diffraction studies. Residual vertex-to-vertex spring lengths remaining, in fact, represent distortions of tetrahedra, whose elastic energy is not accounted for. To rectify this deficiency, topological models can be subjected to molecular dynamics (MD) procedures to refine individual atomic positions without substantially altering the selected topologies. The approach also provides an efficient shortcut of sorts to de novo $\mathrm{MD}$ simulations begun with random atom positions, since the initial modeling obviates simulation routes that will not lead to topologically possible arrangements.

\subsubsection{Initial Configurations and Potentials}

Three forms of collision cascade simulation were initially chosen for MD refinement $[88,89]$ in order to provide three distinguishable model topologies: 1) an $\alpha$ quartz cascade reassembled using $\alpha$-cristobalite assembly rules; 2 ) an $\alpha$-quartz cascade reassembled by maximal global matching; and 3) a $\beta$-cristobalite cascade reassembled by maximal global matching. In each case, an initial crystal of 10,000 tetrahedra was erected, the central 2000 tetrahedra of which were disordered and reassembled. From each of these three reconstructions, a central cube of 500 tetrahedra was selected for subsequent MD refinement. As a reference structure, a randomized silica arrangement was generated by putting $1000 \mathrm{O}$ and $500 \mathrm{Si}$ atoms into random positions in a simulation box; this structure is referred to here as random silica. All four initial configurations were scaled to the same density of $2200 \mathrm{~kg} / \mathrm{m}^{3}$ within a box size of $2.83 \mathrm{~nm}$ on a side.

The two-body van Beest-Kramer-van Santen (BKS) potential function [90] was used because it appears to be on of the best for silicates [48,91,92]. In order to reduce the negative pressure in vitreous silica, a short-range potential cutoff of $0.55 \mathrm{~nm}$ was used, with no noticeable structural change observed. The long-range Coulombic force was calculated using an Ewald summation with a cutoff at $1.2 \mathrm{~nm}$. MD simulations were performed on the topological models using the DL_POLY code [93], with periodic boundary conditions imposed, at selected equilibration temperatures between $500 \mathrm{~K}$ and $5000 \mathrm{~K}$ for $2.5 \times 10^{5}$ timesteps, followed by cooling to $300 \mathrm{~K}$ at a rate of $10 \mathrm{~K} / \mathrm{ps}$. The temperature was scaled down every five timesteps to minimize the impact of thermal changes. Additional simulations with 25,000 steps were performed afterwards at $300 \mathrm{~K}$, and configurations were collected over the last 20,000 steps at intervals of 50 steps for structure analysis. A constant volume and temperature NVT ensemble with the Berendsen [94] algorithm was used throughout the study. The time interval between each timestep was 2 fs for all 14 simulations listed in Table 3. Forcing a high temperature assembly to equilibrate under room temperature density permits the structure to adjust to final density at both high and low temperature, which would not be possible under constant presssure conditions because of the unavoidably high cooling rate used in MD simulations. A more detailed argument for the advantage of using constant volume simulation can be found elsewhere [38,95]. 
Table 3. Bridging oxygen (BO) content, 4-coordinated silicon (Si[4]) content, and potential energies $\left[\mathrm{E}_{\mathrm{pot}}=\mathrm{E}(\right.$ configuration $)=\mathrm{E}($ system $)-\mathrm{E}($ kinetic $\left.)\right]$ for MDrefined silica reconstructions equilibrated at different temperatures $\left(T_{\text {eq }}\right)$

\begin{tabular}{|c|c|c|c|c|}
\hline Structure & $\mathrm{T}_{\mathrm{eq}}(\mathrm{K})$ & $\mathrm{BO}(\%)$ & $\mathrm{Si}[4](\%)$ & $\mathrm{E}_{\text {pot }}(\mathrm{eV} /$ atom $)$ \\
\hline random & 5000 & 99.7 & 99.7 & -19.175 \\
\hline \multicolumn{2}{|c|}{ cristobalite 2500} & 99.8 & 99.6 & - 19.187 \\
\hline \multirow[t]{2}{*}{ match } & 3000 & 99.5 & 99.8 & - 19.194 \\
\hline & 3500 & 99.8 & 100.0 & -19.180 \\
\hline quartz & 2500 & 99.9 & 99.8 & -19.171 \\
\hline \multirow[t]{2}{*}{ match } & 3000 & 99.6 & 100.0 & - 19.191 \\
\hline & 3500 & 99.8 & 100.0 & - 19.179 \\
\hline quartz/ & 500 & 95.9 & 95.8 & - 19.063 \\
\hline \multicolumn{2}{|c|}{ cristobalite 1000} & 98.5 & 99.0 & - 19.108 \\
\hline \multicolumn{2}{|c|}{ reassembly1500 } & 99.1 & 99.6 & - 19.135 \\
\hline & 2000 & 99.4 & 98.7 & - 19.154 \\
\hline & 2500 & 99.8 & 99.6 & - 19.157 \\
\hline & 3000 & 99.8 & 99.6 & - 19.177 \\
\hline & 3500 & 99.9 & 99.8 & - 19.183 \\
\hline
\end{tabular}




\subsubsection{Reassembly Topologies}

MD equilibration resulted in structures that were essentially fully-connected networks of four-coordinated $\mathrm{Si}$ tetrahedra $(>99.6 \% \mathrm{Si}(4)$ at or above $2500 \mathrm{~K}$ equilibration temperature, Table 3). For the partially underconnected quartz/cristobalite reassembly, as the equilibration temperature was increased step-wise from $500 \mathrm{~K}$ to 2000 $\mathrm{K}$, the number of large rings (> 8-rings) decreased steadily (Fig. 8), corresponding to progressive elimination of the underconnection of the initial cascade. At the same time the potential energy of the assembly (represented in Fig. 9 as sets of parallel curves during cooling) decreased monotonically, arresting at 2000-2500 K, then suddenly jumping to a still lower value at $3000-3500 \mathrm{~K}$. The energy jump corresponded to a discrete change in topology, from one dominated by 8-rings with a slightly smaller number of 6-rings to one exhibiting a broad distribution of rings sizes centered at about 7-rings. The same phenomena were observed for MD equilibration of the maximally matched quartz and cristobalite reasssemblies. The former (Fig. 10a) was at first dominated by 8-rings, with a smaller contribution from 6-rings (as in quartz), and the latter (Fig. 10b) by 6-rings alone (as in cristobalite); but above $3000 \mathrm{~K}$ both ring distributions shifted to broad distributions centered between 6- and 7-rings. An MD simulation at $5000 \mathrm{~K}$, starting with random atom coordinates, resulted in a very similar broad distribution, centered between 6- and 7-rings.

MD simulations therefore resulted in three clearly distinguishable structures for disordered silica, graphic representations of which appear in Fig. 11. Although the effective temperature and duration of the reconstructive phase of real cascades in silica are not known, if that temperature were below the glass transition for vitreous silica $\left(T_{g}=\right.$ $1446 \mathrm{~K}$ ) for the major portion of this phase, it is possible that cascade-disordered metamict material may retain some characterizable medium-range structural features of its precursor, or at least of the crystalline polymorph most stable at the effective cascade temperature in the reconstructive phase. Reconstruction of a cascade is also strongly influenced by the structure of the surrounding undamaged material, whose topology overlaps that of the cascade, and the precursor structure will thus continue to exert a structural influence until well after cascades have overlapped. That influence is evident in Fig. 10 and has been confirmed in maximally matched simulations by varying the size of the disordered region. Reconstruction occurring during electron irradiation, which we have shown arises from accumulation of single-atom radiolytic displacements [22] rather than cascades, arguably occurs at the ambient sample irradiation temperature; roomtemperature electron irradiation of silica could, for example, favor more quartz-like metamict topologies (lower temperature polymorph) and on average larger ring sizes because of the denser packing of larger rings. MD-refinement of topological simulations of this metamict pathway is proposed in $\S 5$.

While the near-identical densities $\left(2210 \mathrm{~kg} / \mathrm{m}^{3}\right)$ of vitreous silica and cristobalite argue for similar 6-ring dominated topologies, the higher (by 2.7\%) density of metamict silica $\left(2260 \mathrm{~kg} / \mathrm{m}^{3}\right)$ argues for some larger rings, as noted earlier, and a structure probably more like that of Fig. 11a. The 7-ring centered distribution for the most random form (Fig. 11c) differs from the more 6-ring dominated prediction of the topological arguments for vitreous silica, at least partly because the BKS potential over-represents the Si-Si repulsive interaction. The Si-Si distance in the MD models ( $\sim 0.31 \mathrm{~nm}$ in the 


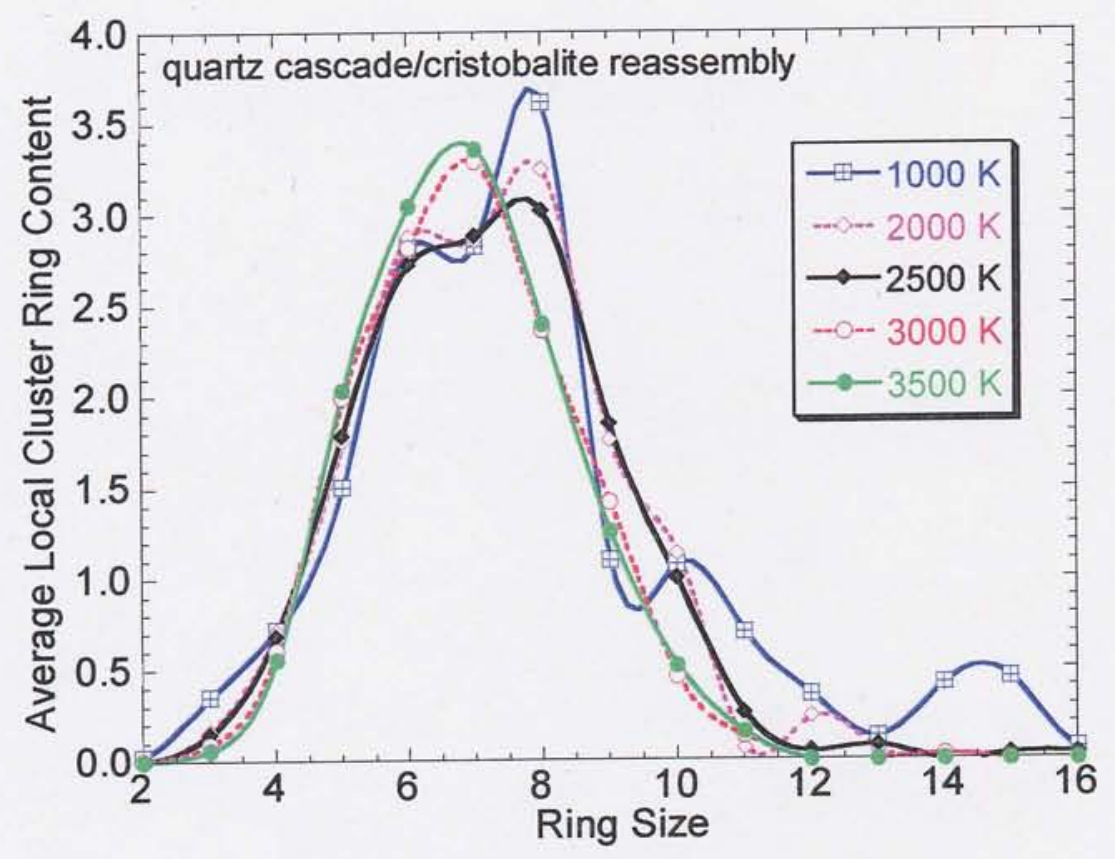

Fig. 8 Distribution of primitive ring content in local clusters of 1500-atom MDrefinement of a 2000 -tetrahedron cascade in $\alpha$-quartz reassembled with $\alpha$ cristobalite rules and equilibrated at successively higher MD simulation temperatures [89].

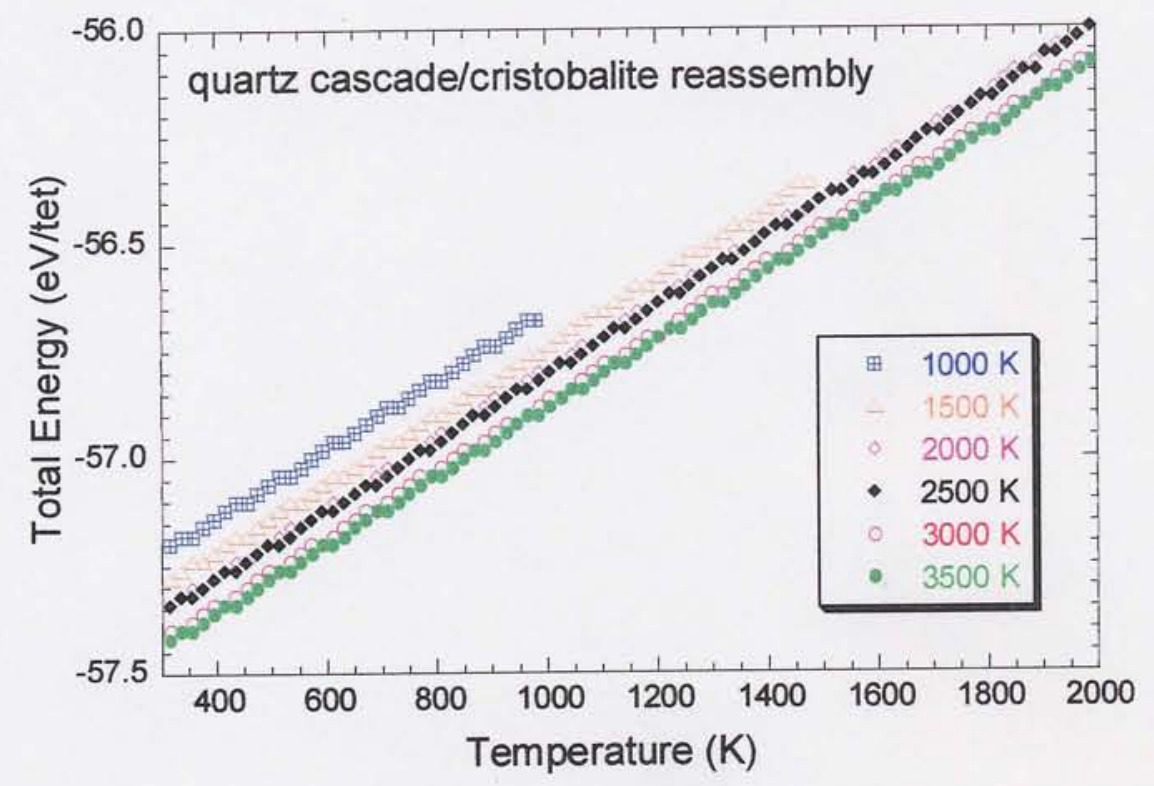

Fig. 9 Evolution of potential energy with increasing MD-refinement temperature for quartz/cristobalite reassembly in Fig. 8 . 

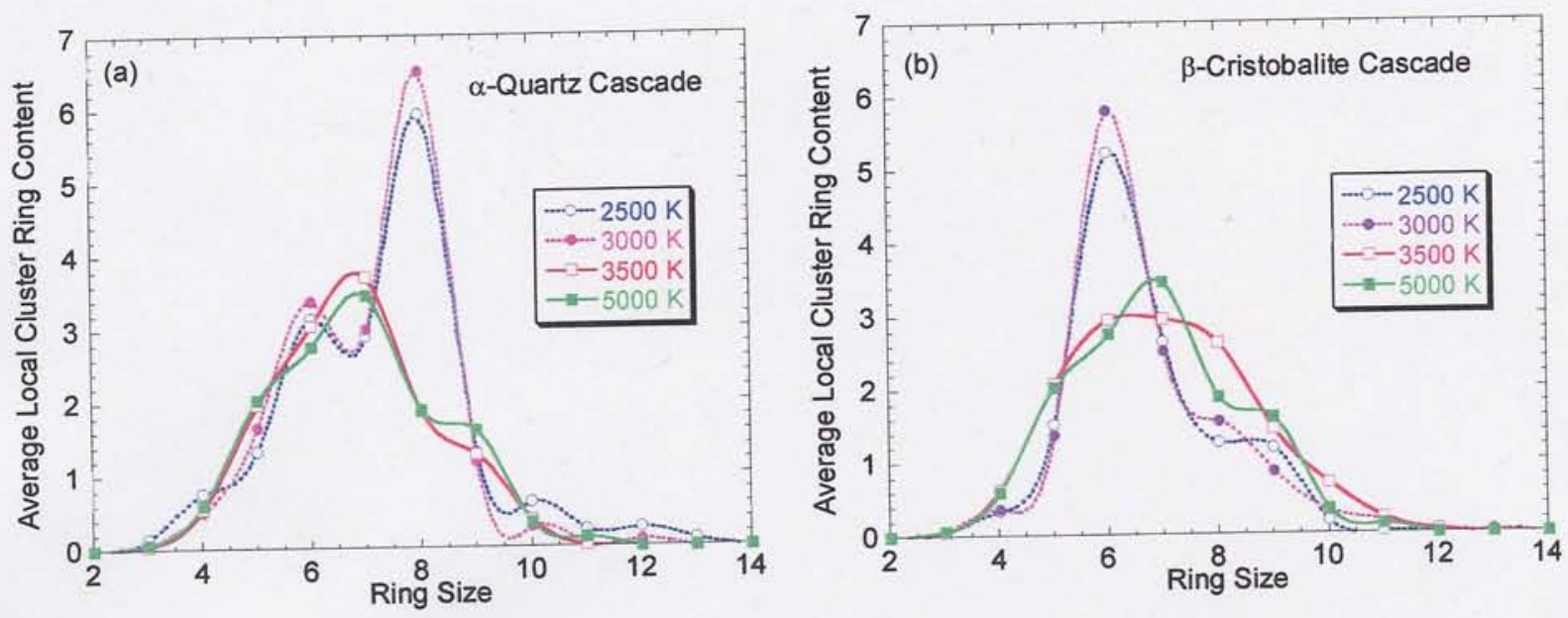

Fig. 10 Distribution of primitive ring content in local clusters of 1500 -atom MD- refinement of a 2000 -tetrahedron maximally matched a) $\alpha$-quartz cascade, and b) $\beta$-cristobalite cascade at three different MD simulation temperatures. The ring distribution of a random silica equilibrated at $5000 \mathrm{~K}$ is plotted as a reference.

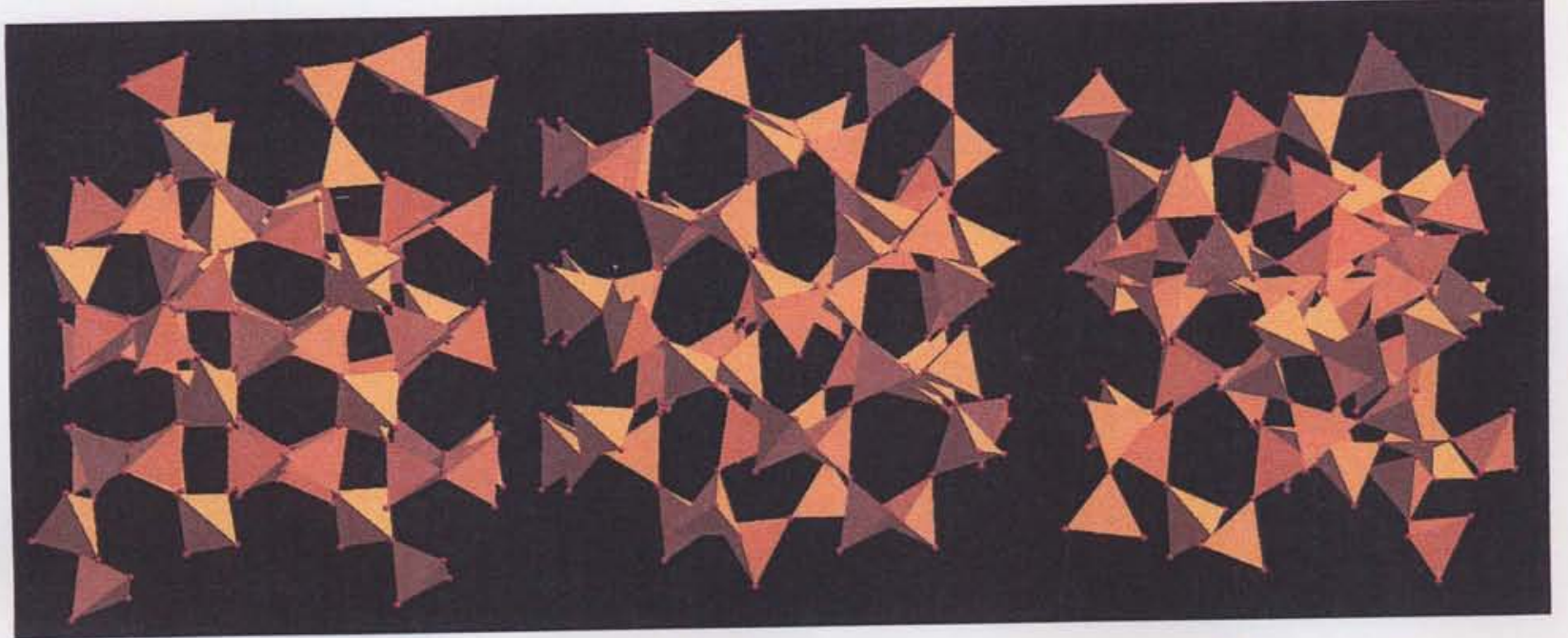

Fig 11 Central portions of 1500 -atom MD-refined a) maximally matched $\alpha$-quartz pseudocascade equilibrated at $2500 \mathrm{~K}$; b) maximally matched $\beta$-cristobalite pseudo-cascade similarly equilibrated at $2500 \mathrm{~K}$, and c) at $3500 \mathrm{~K}$. The latter reverts to a more random structure, topologically similar to that derived from a random initial starting configuration equilibrated at $5000 \mathrm{~K}$. 
correlation function) is distinctly larger than that derived from neutron diffraction for vitreous silica. For the same reason, the Si-O-Si angle distribution for the models is centered at a larger angle angle $\left(\sim 155^{\circ}\right)$ than found for vitreous $\left(\sim 145^{\circ}\right)$ or metamict $\left(\sim 134^{\circ}\right)$ silicas. Some improvement of the potential function in the Si-Si interaction is thus warranted and is also proposed in $\S 5$.

\subsubsection{Real- and Reciprocal-space Correlations}

Their distinctly different topologies not withstanding, the three silica structures of Fig. 11 exhibit remarkably similar real-space correlation functions, shown in Fig. 12a, little different from that of vitreous silica, and with significant differences only well above $0.6 \mathrm{~nm}$. This result is in retrospect not surprising, given that the connectivity of all network silicas is identical at first-neighbor tetrahedra and identical in quartz, cristobalite and tridymite through second-neighbor tetrahedra [85].

Distinctions in medium-range structure are, in fact, better drawn in reciprocal space. In our earlier work [34,97], we have shown that high quality energy-filtered electron diffraction patterns could be obtained using scanning transmission electron microscopy (STEM) and a serial electron energy-loss spectrometer from amorphized silica and SiC specimens. In the current experimental configuration using our VG HB603 300-kV field emission STEM, diffraction information out to scattering vector magnitudes $Q \equiv 4 \pi \sin (\Theta / 2) / \lambda=150 \mathrm{~nm}^{-1}$ can be obtained. (This definition of $Q$ is used in neutron diffraction (ND), but electron diffractionists conventionally prefer to define scattering vectors as $q=2 \sin (\Theta / 2) / \lambda=Q / 2 \pi$, because we make comparisons to ND data, we shall use $Q$ uniformly for both methods.) Electron diffaction data cannot generally compete with neutron diffraction data, which are regularly collected well beyond $Q=400$ $\mathrm{nm}^{-1}$, but most of the additional information collected provides little additional information about intermediate-range structure. Fig. 12b shows that simulated neutron diffraction profiles from the three topologically different silica structures in Fig. 11 are, again, largely indistinguishable throughout almost all of the diffraction profile.

The noteworthy exception occurs at the first diffraction peak [96] — which is fortunately the easiest to collect in electron diffraction (where the dependence of scattering factors on $q$ causes intensities to fall off sharply ( $\sim$ as $\left.q^{-4}\right)$ with increasing diffraction angle, unlike ND where scattering factors are constant with scattering angle). The first peak arises from the longest period real-space correlation; in a crystal, this would be the unit cell-unit cell correlation that gives rise to Bragg peaks, in an amorphous solid the persisting repetition of some characteristic structural idiom. It still remains comparatively sharp in an amorphous solid because the higher spatial frequency Fourier components decay rapidily, so the first diffraction peak is invariably the sharpest. Its greater width for amorphous solids, compared to Bragg peaks from crystals, is related to the decay of the associated Fourier component after a few periods.

Fig. 13 presents data for vitreous silica and three radiation-amorphized quartz samples, obtained with our earlier 100-kV VG HB5 STEM arrangement, from which it is clear that the position of the first diffraction peak differs for these several forms of amorphous silica. (The shift of the second peak for $\mathrm{Si}^{+}$-ion implanted quartz arises from the altered stoichiometry in the amorphized implanted layer.) Fig. 14 depicts an analogous simulated neutron diffraction pattern derived by Fourier transformation from 

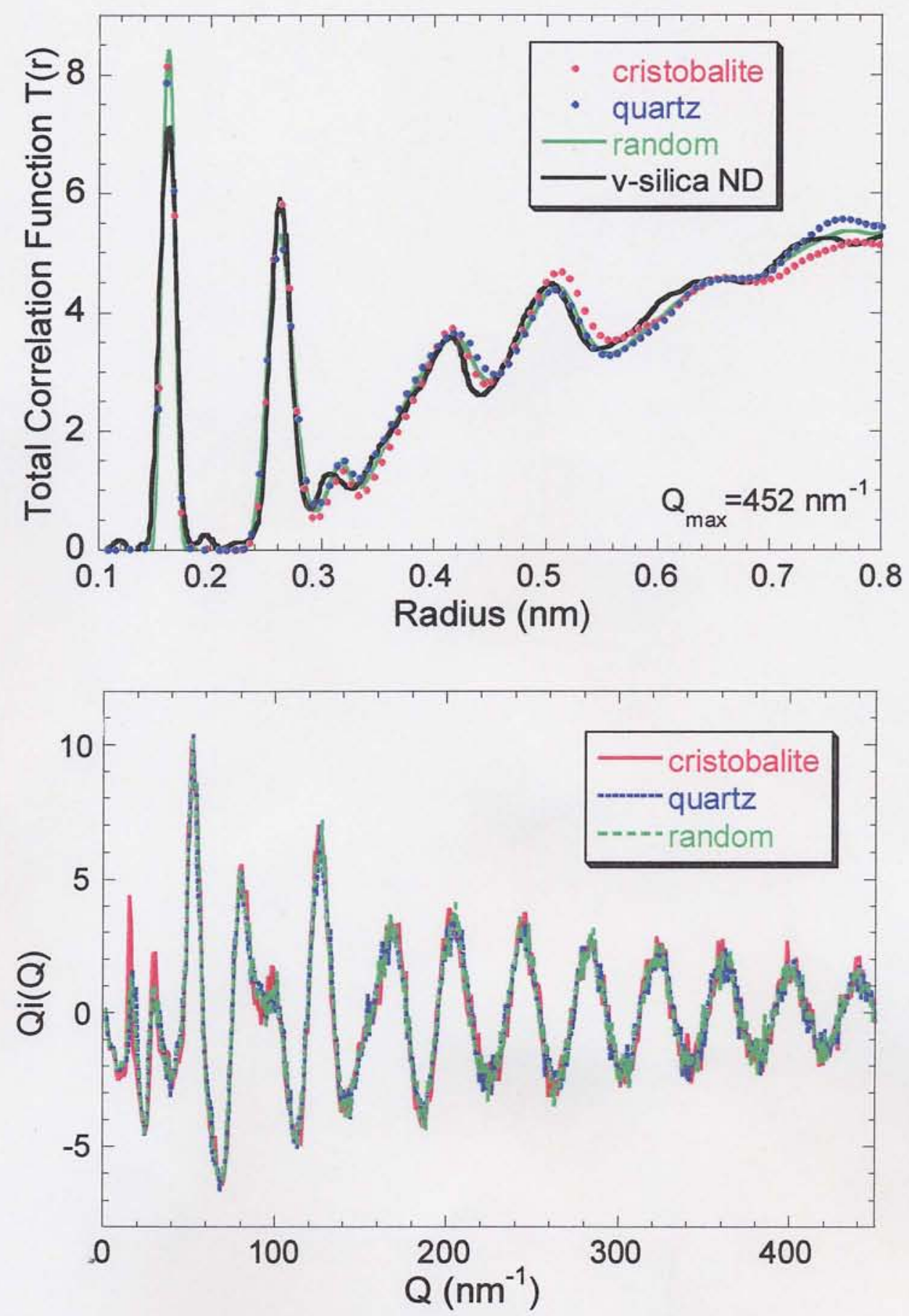

Fig. 12 a) Total real-space correlation functions for the MD-refined quartz and cristobalite cascades and random silica models in Fig. 11, and b) neutron diffraction patterns simulated from (a), showing the indistinguishability of models of very different topologies from overall correlation or diffraction data. 


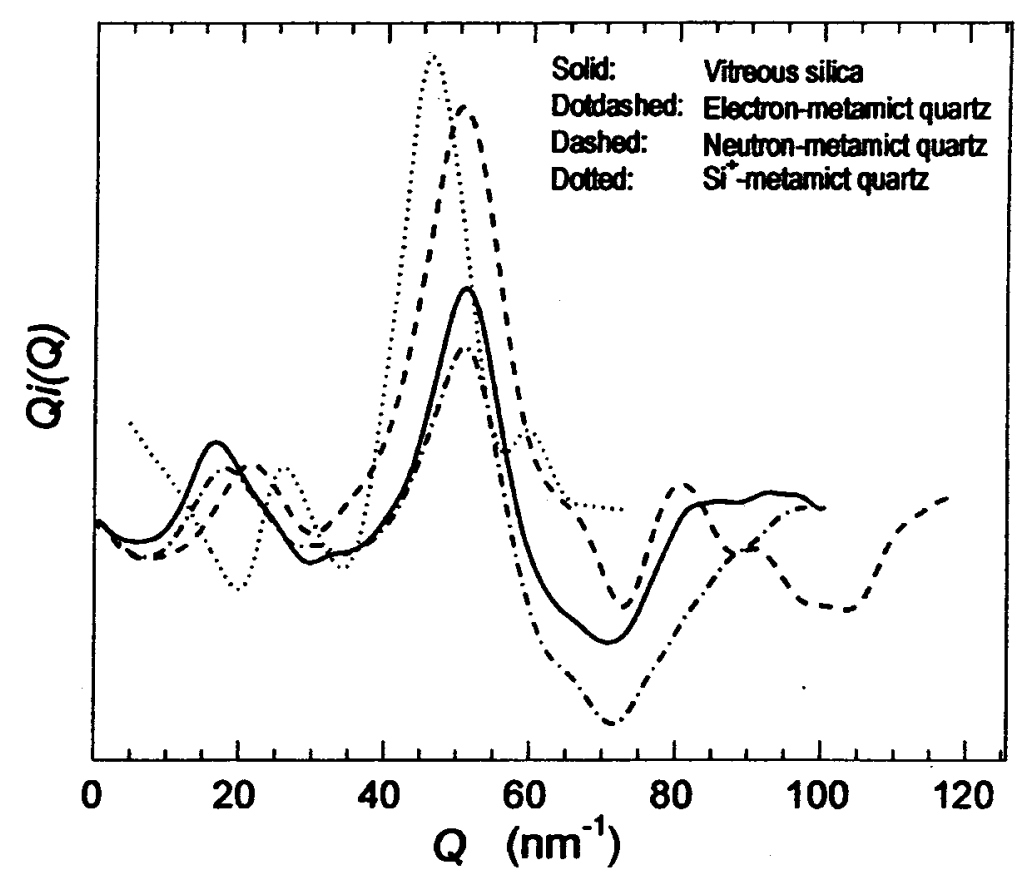

Fig. 13 Experimental angular distribution [34, 97] of scaled electron diffraction interference function $Q i(Q)$ from vitreous silica and $\alpha$-quartz fully amorphized by fast electron, fast neutron, and $\mathrm{Si}^{+}$-ion irradiations, showing characteristic shifts in the position of the first diffraction peak.

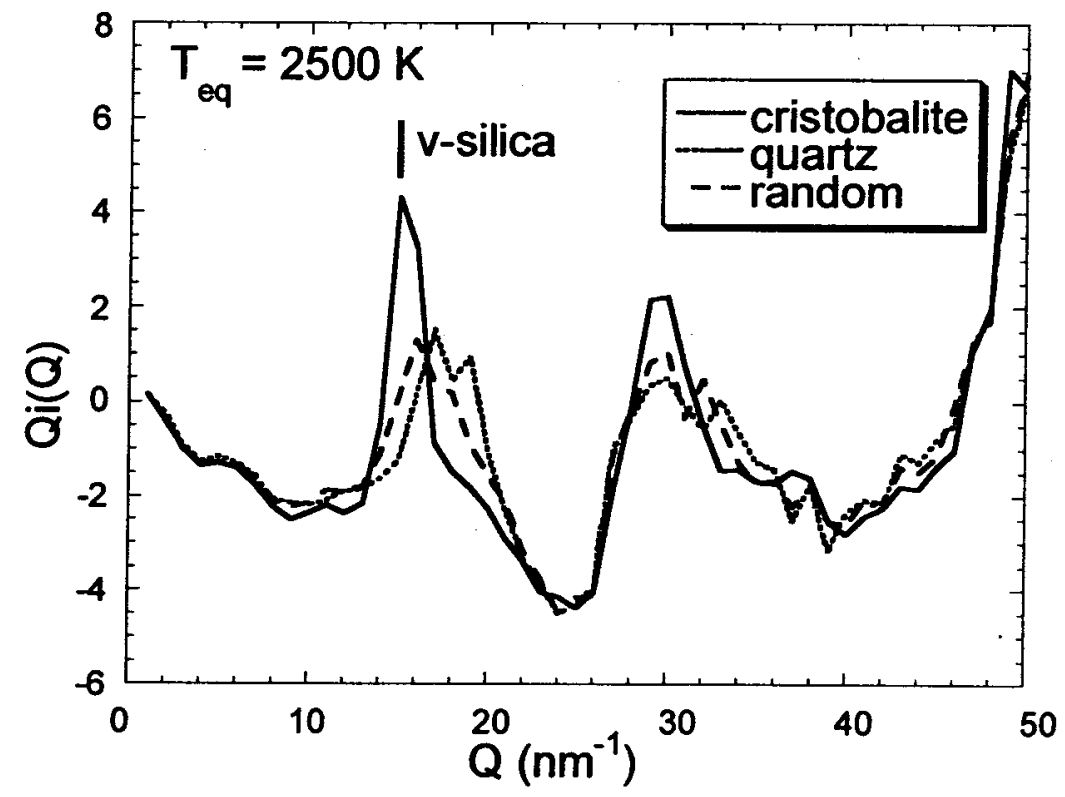

Fig. 14 Simulated neutron-diffraction interference function $[88,89]$ for MDrefined quartz and cristobalite cascades and random silica, showing consistent shifts in the position of the first diffraction peak. 
the MD simulated configurations of Fig. 11, from which a similar conclusion can be drawn. It is clear that structures dominated by larger rings are characterized by first sharp diffraction peaks shifted to higher $Q$, and so to that extent the position of the first diffraction peak is sensitive to topology and appears to be diagnostic. The difference apparent between electron-amorphized and neutron-amorphized quartz in Fig. 13 suggests that different topologies result from cascade-dominated and single displacement-dominated amorphization processes. Certainly, future effort in using electron diffraction (which is the only diffraction technique capable of analyzing the necessarily small volumes of radiation-amorphized metamict material) to explore intermediate-range order should be directed towards producing the best data at the first diffraction peak and not at pushing back the $Q$ limit. We propose this experimental approach in $\S 5$.

\section{MOLECULAR DYNAMICS MODELING OF AMORPHIZED SiC}

Silicon carbide ( $\mathrm{SiC}$ ) is a promising candidate for blanket material of future nuclear fusion reactors, cladding in gas-cooled fission reactors, and inert matrix for transmutation of $\mathrm{Pu}$, as well as for wide band-gap semiconductor devices used in severe environments, such as in military aircraft and combat vehicles, power generation, petrochemical industries, and space. The difficulty of doping SiC by diffusion favor ion implantation routes for electronic device manufacture using this material. Investigations of irradiation damage in SiC and its recovery are thus of considerable technological, as well as scientific, interest [98-102]. Of particular interest has been the irradiationinduced amorphization of SiC [63], which can occur by fast electron [20,78], fast neutron [103] or ion irradiation [29], with a critical amorphization temperature (300-500 K, depending on irradiation type) not far above room temperature. Amorphization appears to occur through accumulation of individual defects or overlap of damage cascades, rather than from direct impact disorder [104].

\subsection{Topology of SiC}

SiC exists in the cubic zincblende structure (3C or $\beta-\mathrm{SiC}$ ) and a large number of polytypes based on the hexagonal wurtzite structure $(6 \mathrm{H}$ or $\alpha-\mathrm{SiC})$. Topologically, they can be viewed as vertex-sharing tetrahedral structures related to other tetrahedral network structures [105]. Fig. 15a illustrates the rules-based self-assembly of $\alpha$-SiC compared to $\alpha$-quartz $\mathrm{SiO}_{2}$ and $\beta-\mathrm{Si}_{3} \mathrm{~N}_{4}$, following the assembly rules for them we have established [44]. Whereas for silicas, two $\left[\mathrm{SiO}_{4}\right]$ tetrahedra join at a vertex and for $\left[\mathrm{Si}_{3} \mathrm{~N}_{4}\right]$ three $\left[\mathrm{SiN}_{4}\right]$ tetrahedra join at a vertex, for $\mathrm{SiC}$ four $\left[\mathrm{SiC}_{4}\right]$ (or $\left[\mathrm{CSi}_{4}\right]$ ) meet at a vertex. The local clusters of $\alpha$ - and $\beta$-SiC both comprise 19 tetrahedra encompassing 12 3-rings and 12 4-rings. Despite the fact that $\beta$-SiC (with its atoms undifferentiated) and Si share the same atom sites, their topologies are quite different because of the need to maintain $\left[\mathrm{SiC}_{4}\right]$ or $\left[\mathrm{CSi}_{4}\right]$ coordination units in $\mathrm{SiC}$. The analogous structural unit for $\mathrm{Si}$ is a single $\mathrm{Si}$ atom with its four tetrahedral bonds extending to bond-midpoint vertices, forming a vertex-sharing $[\mathrm{Si}-4]$ tetrahedron (\$2.2.2). Since ideal $\beta$-cristobalite $\mathrm{SiO}_{2}$ can be thought 


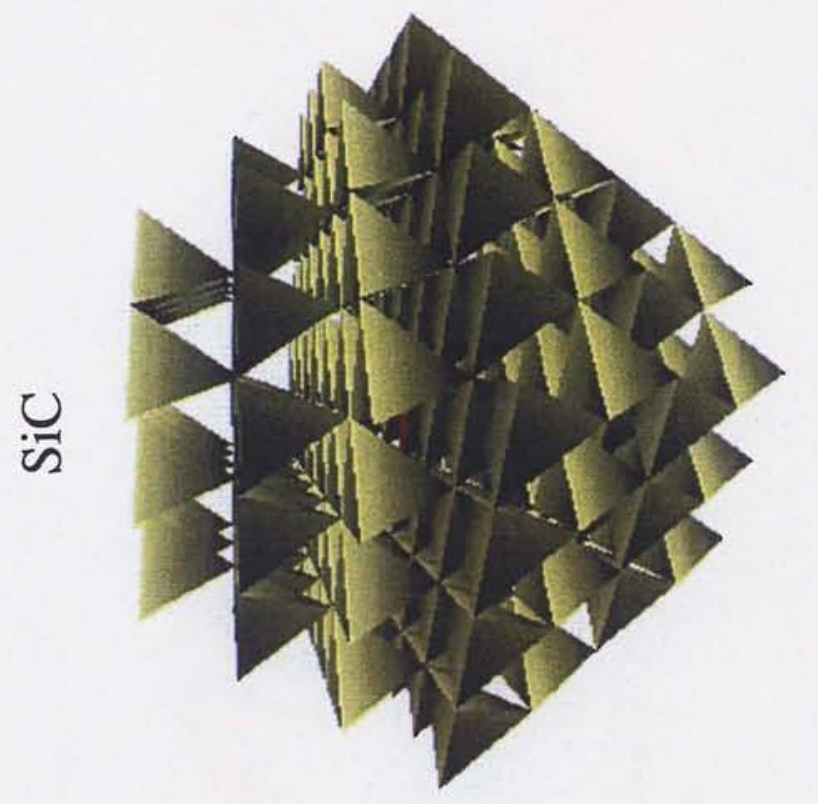

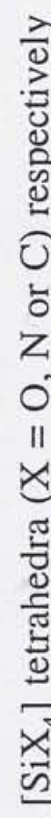
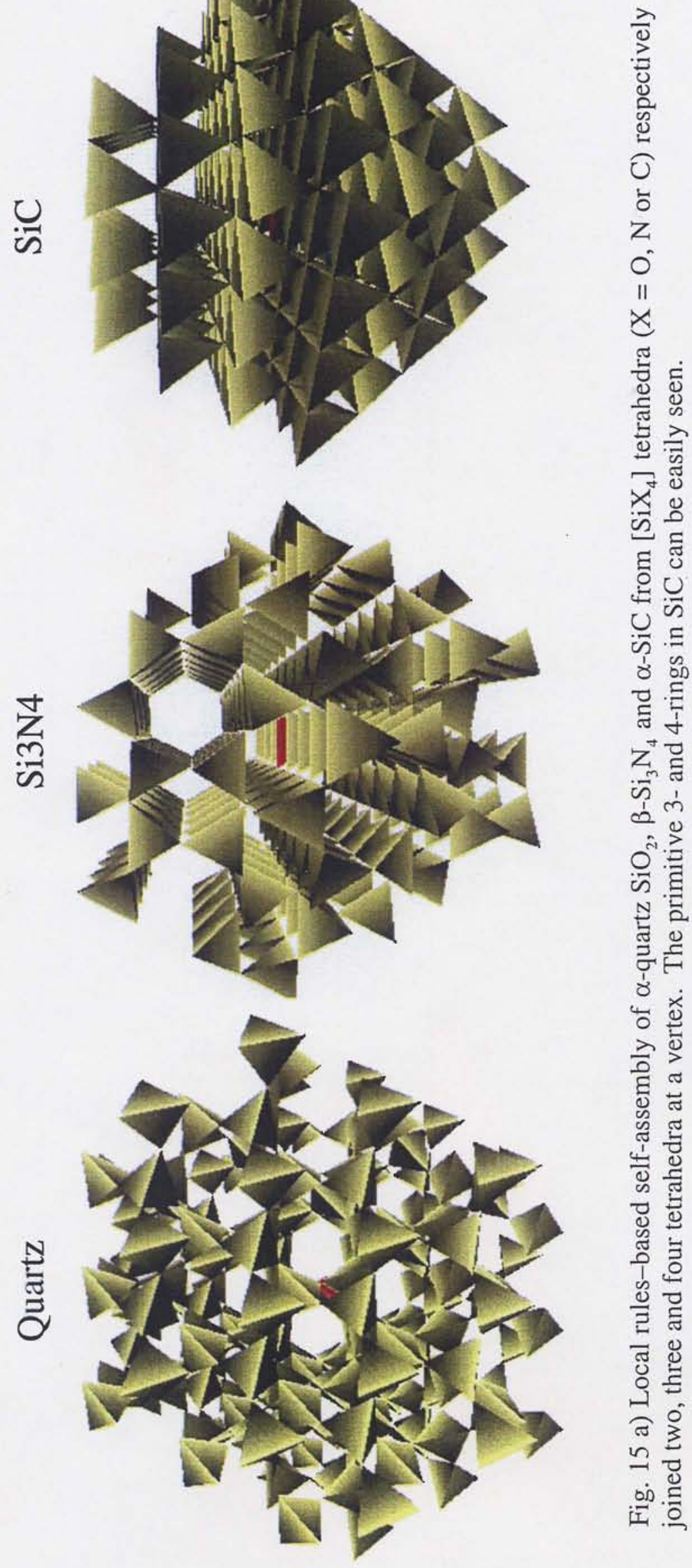





of as the silicon structure with its midpoints decorated by oxygen atoms, Si and cristobalite share the same local cluster topology: 12 6-rings.

\subsubsection{Rigidity and Chemical Disorder}

The large vertex connectivity of $\mathrm{SiC}$ is responsible for the much smaller structural freedom $(f=-3)$ of SiC compared to silicon nitride $(f=-1.5)$ and silica $(f=0)$. In fact, attempts to generate amorphous $\mathrm{SiC}$ structures by disconnection, disordering and reconnection of tetrahedra with their four-connected vertices, as described for silica in §3.1, inevitably meet with failure (Fig. 15b). The fact that $\mathrm{SiC}$ is much more amorphizable (13 eV/atom, Table 1 , not far from $\mathrm{SiO}_{2}$ or $\mathrm{Si}$ ) than suggested by its relative structural freedom has led us to propose repeatedly $[8,10,44,105]$ that the possibility of chemical disorder is responsible for the facile amorphizability of SiC.

The argument runs as follows: The energy required to place Si atoms on $\mathrm{C}$ sites, or $\mathrm{C}$ atoms on Si sites, is small, because both atoms are tetrahedrally coordinated and do not differ significantly in size. In fact, it is slightly easier to place $\mathrm{C}$ atoms on Si sites than vice versa [41], as expected from size considerations. During irradiation, replacement collisions sequences or recombination of interstitials of one sublattice with vacancies of the second leads to mixing of the two sublattices. If the chemical disorder proceeded to the point of random site occupation, then $\beta$-SiC would become topologically indistinguishable from silicon (for which $f \sim 0$ ) with an average atom type and ought to be more readily amorphizable on topological grounds, as argued in $§ 2.3 .2$ Hence, some threshold chemical disorder should be one requirement for amorphization.

As indicated earlier, there is, in fact, experimental evidence from Raman and EXAFS measurements [75-77] that significant chemical disorder attends low-temperature ion irradiation of $\mathrm{SiC}$. Various MD simulations of SiC cascades and cascade overlap [39$41,74,104,106-110,123$ ] have also confirmed the resulting chemical disorder, but its enumeration has been carried out in terms of antisite defects based on occupation of Wigner-Seitz cells centered on original reference lattice atom sites. Antisite defects were, however, found to be less numerous (typically, $<10 \%$ of the defects produced) than Frenkel defects, whose accumulation has instead been primarily supposed to trigger amorphization.

The exclusive focus on the formal antisite configuration itself has ignored the multiplier effect of atom interchange on the immediate coordination of a much larger number of atoms and hence on the ability to define tetrahedral units which underpin the principal structural constraint. Replacing a $\mathrm{C}$ atom with a Si atom on the carbon atom site generates an antisite configuration (the Si replacement forming four homonuclear SiSi bonds), but each of the four surrounding Si atom sites is then anomalous also, with three heteronuclear bonds and one homonuclear bond. Recent MD simulation of cascade displacements [108] suggests that most interstitials-in at least low-energy displacements - are in Si-C and C-C dumb-bell interstitial configurations (respective formation enthalpies $3.3 \mathrm{eV}$ and $3.2 \mathrm{eV}$ [111]), so that even Frenkel disorder can result in homonuclear bonds which contribute topologically to chemical disorder.

As chemical disorder accumulates, the increasingly more appropriate analysis is homonuclear bond enumeration, which more accurately reflects the changing topology. A well-separated $\mathrm{C}$ antisite and $\mathrm{Si}$ antisite pair, in isolation, together generate four Si-Si 
and four $\mathrm{C}-\mathrm{C}$ homonuclear bonds, replacing eight normal heteronuclear bonds; when they occupy adjacent sites, each defect is associated with three homonuclear bonds (three Si$\mathrm{Si}$ and three $\mathrm{C}-\mathrm{C}$ respectively) and one normal Si-C bond. An interstitial $\mathrm{C}$ atom (the more numerous interstitial defect [41]), forming a single, split or dumb-bell interstitial results in several possible homonuclear and several heteronuclear bonds, but with no formal antisite defect generated. As long-range order is lost and the ability to unambigously identify sites with a crystalline reference lattice diminshes, antisite configurations could continue to be defined topologically from site coordination (e.g. a Si atom surrounded by four other Si atoms); but their enumeration then becomes only a more restrictive form of homonuclear bond enumeration, and their identification fails once there is significant overlap of antisite coordination shells. Hence, where there is significant overlap of antisite defects, where there is a significant number of interstitial atoms that are accommodated, or once a crystalline reference lattice has been lost to amorphization, homonuclear bond enumeration is the only form of analysis which is meaningful to characterize the chemical disorder.

\subsubsection{Chemical and Topological Disorder Metrics}

In characterizing the topology of disordered solids, two metrics are useful: the coordination number (CN) and the local cluster primitive ring content (LC). Given the possibility of chemical disorder, in our work CN were enumerated without regard to atom type. Similarly, the structural unit chosen to describe the ring topology was an undistinguished atom (and its bond midpoints), and for this choice the LCs for crystalline $\alpha$ - or $\beta$-SiC comprise 12 6-rings, as indicated above. The LC ring-content statistics for the MD simulations described below were obtained by utilizing a highly efficient ring search algorithm [112]. To characterize the chemical disorder, three further parameters are commonly employed: chemical disorder parameter $\chi$, short-range order parameter $\sigma$, and Bragg-Williams long-range order parameter $\lambda$.

Chemical disorder specifically involves atom chemical identities, but it is more instructive for reasons which will become apparent to focus on the bond identities (C-C, $\mathrm{Si}-\mathrm{Si}$ or Si-C bonds). In perfect $\mathrm{SiC}$, only heteronuclear bonds (Si-C) exist. Homonuclear bonds (C-C, Si-Si) are formed when antisite or Frenkel defects are introduced. The homonuclear bond ratio $R_{h n b}$, defined for $\mathrm{SiC}$ as the ratio of number of homonuclear bonds to twice the number of heteronuclear bonds, provides a full homonculear bond analysis. For perfect $\mathrm{SiC}, R_{h n b}=0$, while for a chemically random lattice $R_{h n b}=1$. Chemical disorder $\chi$, defined as the ratio of C-C bonds to C-Si bonds, $\mathrm{N}_{\mathrm{C}-\mathrm{C}} / \mathrm{N}_{\text {Si-C }}$ [113], is not a complete homonuclear bond analysis and is specified only for $\mathrm{C}$ atoms. The argument for using $\chi$ instead of $R_{h n b}$ derives mostly from the practical uncertainty of enumerating Si-Si bonds in amorphous structures for which Si atoms have no clearly defined first coordination shell [112-114]. In practice, $\chi$ is quite a good approximation to $R_{h n b}$, and the deviation is proportional to the CN difference between $\mathrm{Si}$ and C; when all atoms have the same $\mathrm{CN}, \chi=R_{h n b}$.

The short-range order parameter proposed by Gehlen and Cohen [115], $\sigma=1-$ $p_{\mathrm{AB}} / x_{\mathrm{A}}$, where $p_{\mathrm{AB}}$ is the probability of finding an $\mathrm{A}$ atom in the nearest-neighbor coordination shell around a $\mathrm{B}$ atom and $x_{\mathrm{A}}$ is the atom fraction of A atoms, is merely

another form of homonuclear bond analysis. With an assumption that all $\mathrm{N}_{C} \mathrm{C}$ atoms 
have four nearest neighbors, a normalized short-range order parameter for perfect $\mathrm{SiC}$ can be expressed [74] as $\sigma=\mathrm{n}_{\mathrm{Si}-\mathrm{C}} / 2-1$, where $\mathrm{n}_{\mathrm{Si}-\mathrm{C}}=\mathrm{N}_{\mathrm{Si}-\mathrm{C}} / \mathrm{N}_{\mathrm{C}}$ is the average number of nearest-neighbor Si atoms surrounding a $\mathrm{C}$ atom. Use of $\chi$ and $\sigma$ is then equivalent, with their equivalence relationship given by $\chi=(1-\sigma) /(1+\sigma)$.

The Bragg-Williams long-range order parameter [116] for a binary compound $\mathrm{AB}, \lambda=1-p\left(\mathrm{~A}_{\mathrm{B}}\right) / x_{\mathrm{B}}$, where $p\left(\mathrm{~A}_{\mathrm{B}}\right)$ is the probability of finding an A atom on a B site and $x_{\mathrm{B}}$ is the atom fraction of $\mathrm{B}$ atoms, requires prior identification of fixed sites associated with a specific atom type, as defined by a reference lattice. For SiC, it can therefore be expressed [74] as $\lambda=1-2 \mathrm{~N}_{\text {anti }} / \mathrm{N}$, where $\mathrm{N}_{\text {anti }}$ is the total number of antisites (viz Si atoms on sites pre-labeled as $\mathrm{C}$ sites and vice versa) and $\mathrm{N}$ is the number of atoms in the crystal. Obviously, $\lambda$ is simply a normalized antisite analysis, and for the same reason as the inability to define antisite discussed above, the formal Bragg-Williams scheme fails entirely once a reference lattice is lost or there is significant overlap of antisite coordination shells.

\subsection{Molecular Dynamics Simulations of Disorder}

As for $\mathrm{SiO}_{2}, \mathrm{MD}$ simulations provide a useful experimental platform on which to explore topological ideas. MD simulations were again performed with the DL_POLY computational code [93] and the Berendsen algorithm [94], using a 2 fs timestep throughout. Three classes of simulation were carried out: 1) equilibration from initially random atom positions at high temperature ( $5000 \mathrm{~K}$ ), followed by a quench; 2) evolution of an embedded pseudo-cascade from random atom positions at $2500 \mathrm{~K}$; and 3) equilibration of an initially translationally perfect $\beta$-SiC assembly with selected introduction of chemical disorder.

\subsubsection{Choice of Potential}

Appropriate choice of interatomic potential was found to be particularly important [117] in our SiC simulations. Three different three-body potential forms developed by Tersoff were explored, developed in 1989 [118], 1990 [119] and 1994 [113] respectively. These have been found [120] to be more appropriate for modeling SiC than the Pearson [121] and modified EAM [122] potentials. The consequences of using the different forms of the Tersoff potentials was explored by generating amorphous $\mathrm{SiC}$ structures by high-temperature MD followed by a quench. Initial simulation cells consisted of $864 \mathrm{Si}$ and $864 \mathrm{C}$ atoms randomized in a $6 \mathrm{a}_{0} \times 6 \mathrm{a}_{0} \times 6 \mathrm{a}_{0}\left(\mathrm{a}_{0}=0.436 \mathrm{~nm}\right)$ box with periodic boundary conditions imposed. Atoms in the cells were allowed to equilibrate at $5000 \mathrm{~K}$ for $400 \mathrm{ps}\left(2 \times 10^{5}\right.$ time steps) before cooling down to $300 \mathrm{~K}$ at $10 \mathrm{~K} / \mathrm{ps}$. Subsequent simulation was then carried out at $300 \mathrm{~K}$ for a further $10 \mathrm{ps}$ (5,000 timesteps), and configurations were collected over the last 1,000 timesteps, at intervals of 50 timesteps, for structure analysis. Table 4 indicates coordination numbers, homonuclear bond ratios, chemical disorder $\chi$, and disorder enthalpy $\Delta H$ for the a-SiC assemblies thus generated with the three potentials, and Fig. 16 the corresponding total pair correlation functions $g(r)$. 
Table 4. Coordination numbers,homonuclear bond ratios, chemical disorder and disorder enthalpy in a-SiC modeled using different Tersoff potentials. Data in parentheses are from [113].

\begin{tabular}{ccccccc}
\hline Year & Si/C CN & Si-Si/Si-all & C-C/C-all & $\chi[=\mathrm{C}-\mathrm{C} / \mathrm{C}-\mathrm{Si}]$ & $\Delta H(\mathrm{eV} / \mathrm{bond})$ & Notes \\
\hline 1989 & $5.0 / 3.7$ & 0.54 & 0.37 & 0.59 & -0.09 & $\pi$-bonded C \\
1990 & $3.9 / 4.0$ & 0.23 & 0.24 & 0.32 & -0.43 & Poor PDF \\
1994 & $4.8 / 4.0$ & 0.47 & $0.36(0.33)$ & $0.56(0.50)$ & $-0.12(-0.18)$ & a-SiC \\
\hline
\end{tabular}




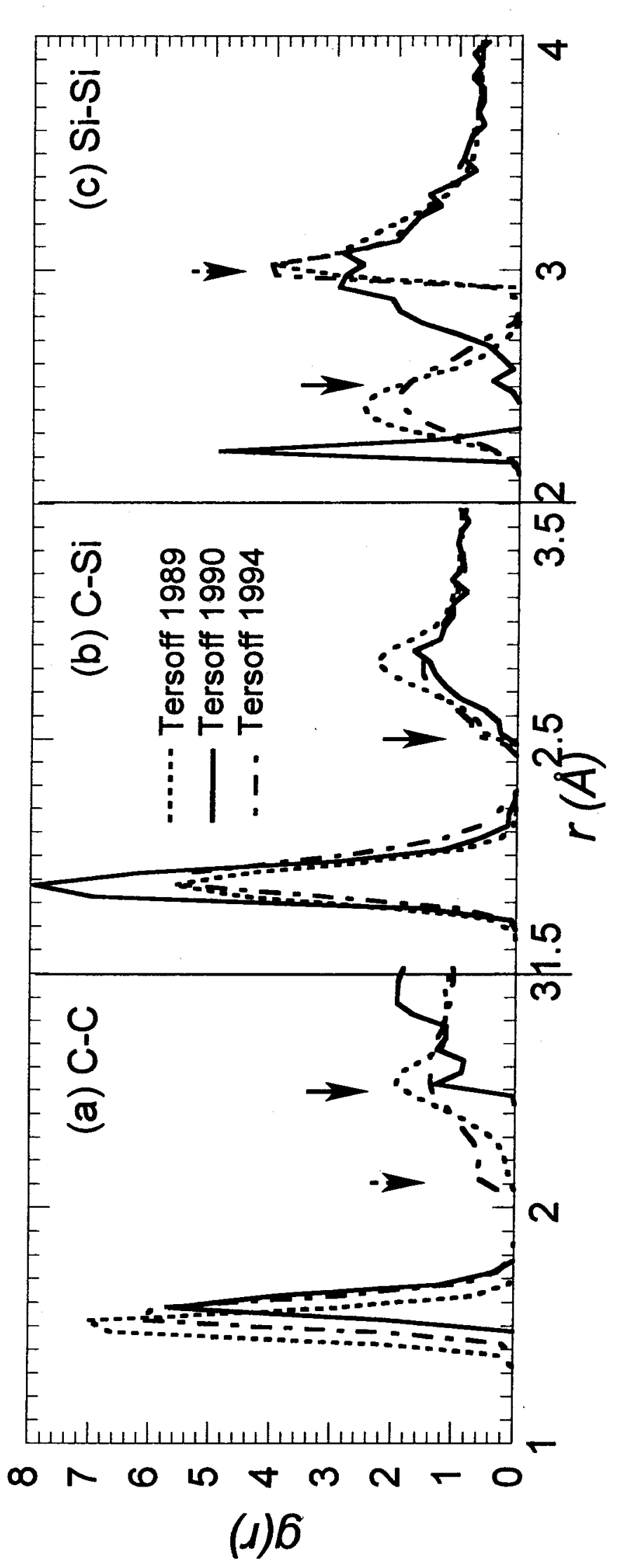

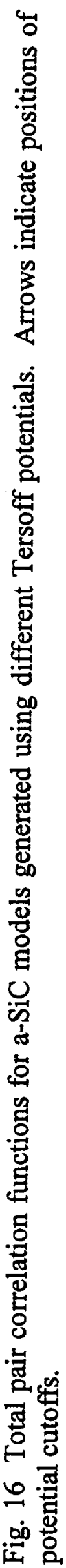


The Tersoff 1989 potential generated a noticeable quantity of 3-fold $\mathrm{C}$ atoms and thus gave an average $\mathrm{C}$ coordination number $\mathrm{CN}=3.7$. (We define coordinated atoms as those within the first peak before the first minimum in $g(r)$.) The smaller $\mathrm{CN}$ of $\mathrm{C}$ arises from C $\pi$-bonding, as in graphite. The 1990 and 1994 potentials were modified from the 1989 potential to give an increase in $\mathrm{CN}$ for $\mathrm{C}$ atoms in a-SiC, as indicated in Table 4. However, the main structural differences arise from the different potential cutoffs used in the 1990 and 1994 potentials. While Tersoff has indicated [119] that the abrupt truncation at $2.5 \AA$ is unphysical and inappropriate for unconstrained dynamical simulations, its use does give a well-defined Si local structure (Fig. 16c) and "ideal" CN near 4 for both Si and C atoms (Table 4). There is, nevertheless, much reduced chemical disorder $\chi$ due solely to the change in potential cutoff. The aversion to forming homonuclear bonds results from the $2.5 \AA$ cutoff being situated squarely in the center of the first Si-Si pair-correlation peak (Fig. 16c); this cutoff pushes many Si atoms away from central $\mathrm{Si}$ atoms and thus leaves room for $\mathrm{C}$ atoms to form more $\mathrm{Si}-\mathrm{C}$ bonds, as seen in Fig. 16b. Because Si-C bonds are shorter than Si-Si bonds, a reduction in the $\mathrm{CN}$ of $\mathrm{Si}$ atoms is not surprising. Furthermore, the increase in $\mathrm{Si}-\mathrm{C}$ bonds also results in more $\mathrm{Si}$ atoms around $\mathrm{C}$ sites and concomitantly reduces the possibility of forming $\mathrm{C}-\mathrm{C}$ homonuclear bonds. Whenever the $\mathrm{CNs}$ of $\mathrm{Si}$ and $\mathrm{C}$ become equal, the $\mathrm{Si}-\mathrm{Si}$ homonuclear bond ratio (Si-Si/Si-all bonds) must be equal to the $\mathrm{C}-\mathrm{C}$ homonuclear bond ratio (C-C/C-all bonds).

Clearly, choice of cutoff has significant impact on the probability of forming homonuclear bonds. The energy barrier for chemical disorder can be roughly estimated [113] by $\left.\chi=\exp \left[(\Delta H+\varepsilon) / k T^{*}\right)\right]$, where the enthalpy $\Delta H$ is the energy of a Si-C bond relative to the average $\mathrm{Si}-\mathrm{Si}$ and $\mathrm{C}-\mathrm{C}$ bond energies, $\varepsilon$ is the contribution from the atomic size difference (about $0.2 \mathrm{eV} / \mathrm{bond}$ ), and $T^{*}$ is an effective temperature about $6000 \mathrm{~K}$. Table 4 demonstrates that using a $2.5 \AA$ cutoff (Fig. 16b) more than doubles $\Delta H$, disfavoring formation of homonuclear bonds. By contrast, the smooth cutoffs in the 1989 and 1994 potentials yielded higher homonuclear bond counts, as well as also about 50\% 5-coordinated Si and non-negligible numbers of 6- and even 7-coordinated Si. The presence of these highly-coordinated Si atoms significantly changes the topology of a$\mathrm{SiC}$ structures. In particular, the local-cluster ring distributions for both $\mathrm{Si}$ and $\mathrm{C}$ origins (which are centered near 6-rings) are shifted towards slightly smaller ring size, probably because the highly-coordinated $\mathrm{Si}$ atoms provide shortcuts to the larger rings present. The 1994 Tersoff interatomic potential was chosen as the most physically realistic in all subsequent simulations.

\subsubsection{Pseudo-Cascade Embedded Simulations}

A set of simulations was first carried out on a SiC pseudo-cascade comprising 512 Si and $C$ atoms, within a $\left(4 \mathrm{a}_{0} \times 4 \mathrm{a}_{0} \times 4 \mathrm{a}_{0}\right)$ simulation block of 256 unit cells $\left(\mathrm{a}_{0}=0.436 \mathrm{~nm}\right)$ embedded in a surrounding perfect $\beta$-SiC matrix, whose positions were originally randomized [117]. MD simulations were subsequently carried out at $2500 \mathrm{~K}$ for $10 \mathrm{~ns}$ $\left(5 \times 10^{6} 2\right.$-fs time steps) to re-coordinate the atoms. The resulting reconstruction, whose evolution at $2500 \mathrm{~K}$ is shown in Fig. 17, remained amorphous. It was compared to a lowenergy recoil (100 eV) 512-atom MD cascade, recently simulated by Malerba and 


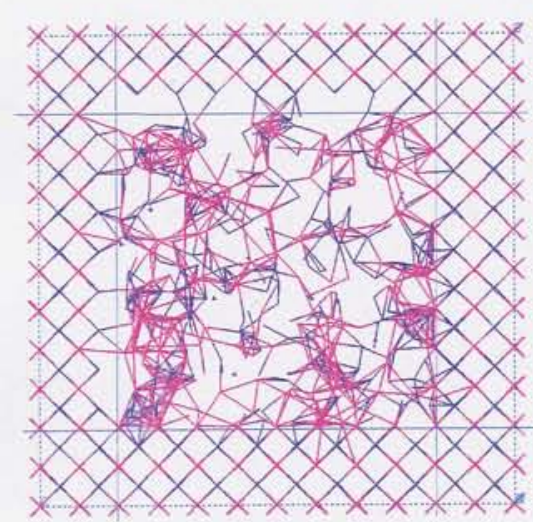

(a) $0 \mathrm{~ns}$ (0 step)

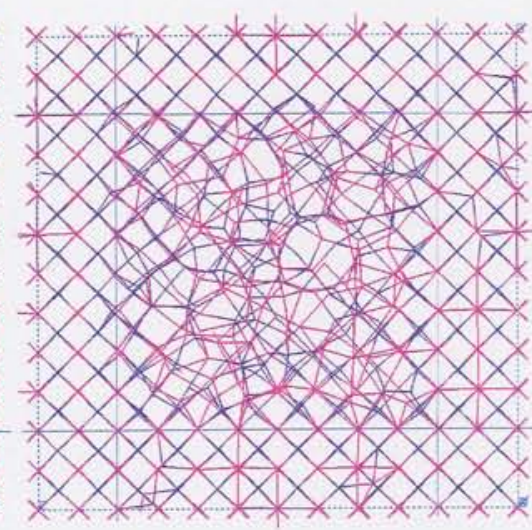

(b) $2 \mathrm{~ns}\left(1 \times 10^{6}\right.$ steps $)$

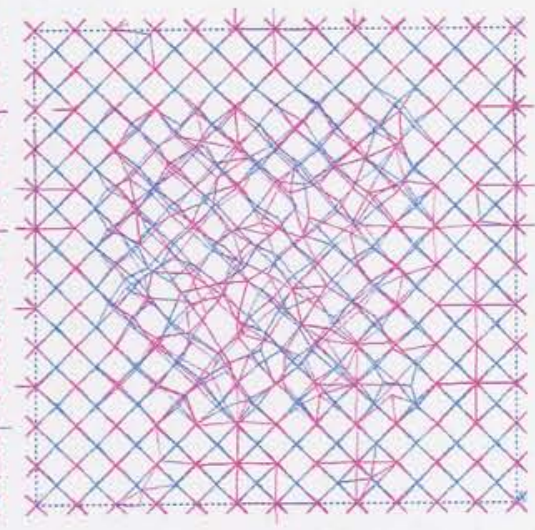

(c) $10 \mathrm{~ns}\left(5 \times 10^{6} \mathrm{steps}\right)$

Fig. 17 Evolution of embedded pseudo-cascade in $\beta$-SiC from random atom starting positions at $2500 \mathrm{~K}$ using the 1994 Tersoff potential. Pink nodes are $\mathrm{Si}$ atoms, blue nodes $\mathrm{C}$ atoms.

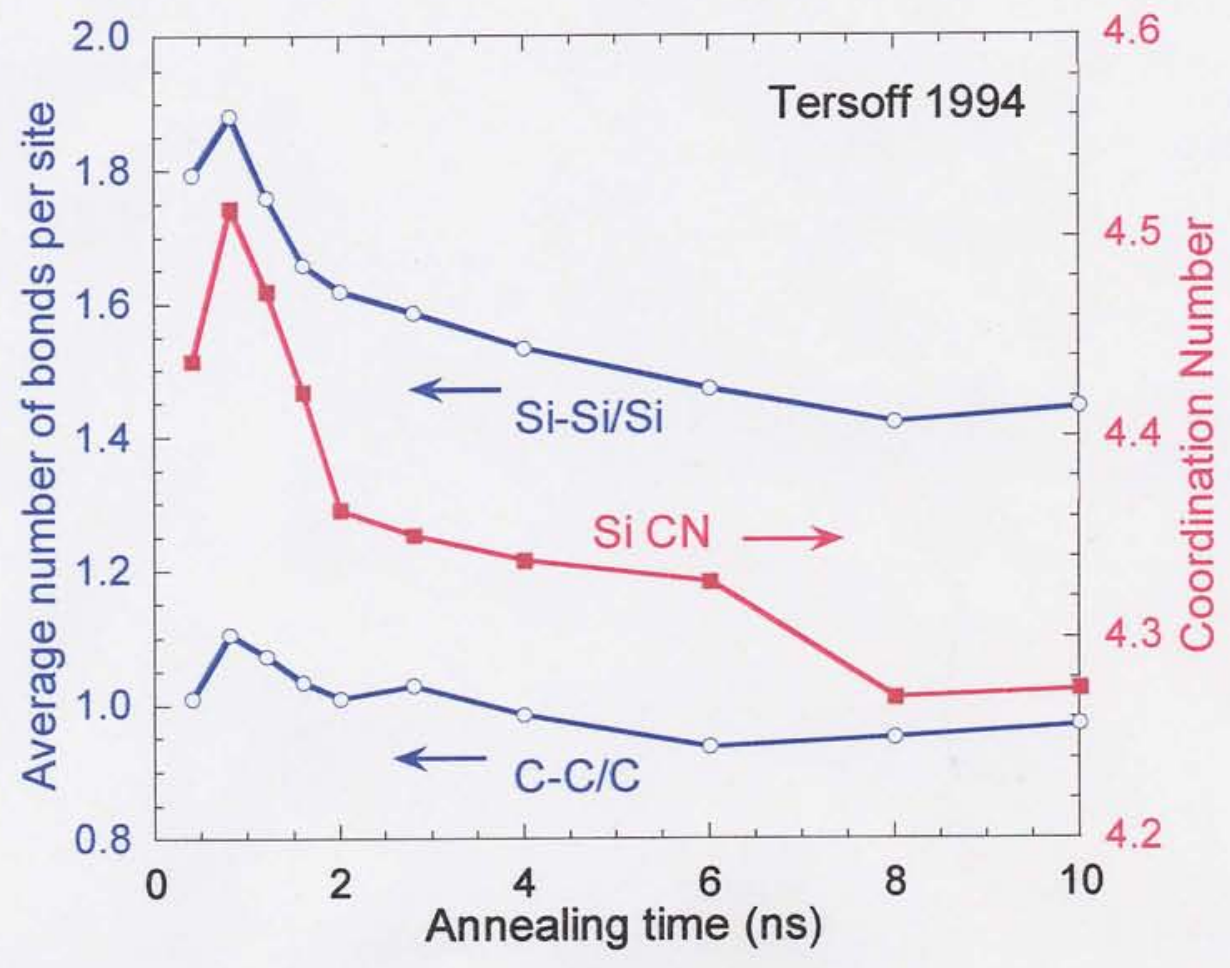

Fig. 18 Average homonuclear bond fraction per site and silicon coordination number $(\mathrm{CN})$ as a function of annealing time at $2500 \mathrm{~K}$ for embedded pseudocascade in Fig. 17. 
Perlado [123] at a temperature of $20 \mathrm{~K}$ using the 1990 Tersoff potential [119] and subsequently annealed at $2320 \mathrm{~K}$ for $25 \mathrm{~ns}$, after which it had effectively recrystallized.

An enumeration (Fig. 18) of the still-amorphous end-state, embedded reconstruction in Fig. 17 revealed evolution to an average Si coordination number of 4.27 at steady-state (10 ns) and an average of 1.45 homonuclear bonds per Si and 1.0 per C atom $(\chi=0.32)$ [117]. Fig. 19 shows that both this reconstruction and the MalerbaPerlado annealed cascade [123], while exhibiting a very small number of topological antisite defects ( $\mathrm{C}$ atoms with four homonuclear bonds), nevertheless contained a wide distribution of more numerous partial antisites (sites with 1, 2 or 3 homonuclear bonds). Both enumerations included the boundary between initially disordered and surrounding crystalline material; the number of normally coordinated sites $\left(\mathrm{SiC}_{4}, \mathrm{CSi}_{4}\right)$ is larger nearer the boundary and demonstrates the role of the surrounding matrix in inducing epitaxial recrystallization. The initial Malerba-Perlado low-temperature (20 K) metamict cascade exhibited a chemical disorder of $\chi=0.27$, while for their final recrystallized annealed cascade $\chi=0.22$. The former value is lower than that $(\chi=0.34)$ calculated from the $\sigma=0.488$ short-range order parameter supplied by Malerba and Perlado, which must not have included the boundary count.

In their simulation, Malerba and Perlado found that during annealing the number of antisites increased as the metamict region regained its periodicity. (By way of contrast, simulations by Gao and Weber [74] of SiC displacement cascades initiated by $10 \mathrm{keV} \mathrm{Si-} \mathrm{or} \mathrm{Au-ion} \mathrm{recoils} \mathrm{found} \mathrm{that} \mathrm{amorphous} \mathrm{regions} \mathrm{of} \mathrm{their} \mathrm{cascades} \mathrm{had} \mathrm{higher}$ antisite concentration than other regions of the cascade.) Malerbo and Perlado accordingly discounted the role of antisites in $\mathrm{SiC}$ amorphization and concluded that accumulation of Frenkel defects was mainly responsible. In their analysis, as indicated previously, antisites were obtained by comparing coordinates of current atom locations with those of their original sites in the perfect structure; this procedure, while acceptable for undistorted crystalline structures with few antisites, provides the wrong metric for assessing more chemically disordered configurations, and is inappropriate for topologically disordered structures. Furthermore, our homonuclear bond analysis of their structures revealed that the annealed structure had a lower value $(\chi=0.22)$ than the original metamict configuration $(\chi=0.27)$. Excluding the boundary, the disparity is even greater $(\chi \approx 0.35$ vs. $\chi \approx 0.5$ ). Thus, their modeling result in fact supports the present argument that homonuclear bond density correlates with the ability to sustain topological disorder.

The persistence of crystallinity in the Malerba-Perlado simulation is, we believe, attributable to two factors: the low recoil $(100 \mathrm{eV})$ energy employed, which more closely approximates electron irradiation conditions than ion or neutron irradiation; and the use of the 1990 Tersoff potential. The chemical disorder in their simulation never reaches levels high enough to strongly favor retention of the amorphous state.

\subsubsection{Chemical Disorder Simulations}

A third set of simulations, again using the 1994 Tersoff potential, utilized as starting configurations seven initially translationally perfect (hence topologically ordered) $\beta$-SiC crystal structures with varying imposed levels of chemical disorder [124]. Each initial structure consisted of $864 \mathrm{Si}$ and $864 \mathrm{C}$ atoms in a $6 \mathrm{a}_{0} \times 6 \mathrm{a}_{0} \times 6 \mathrm{a}_{0}$ simulation 

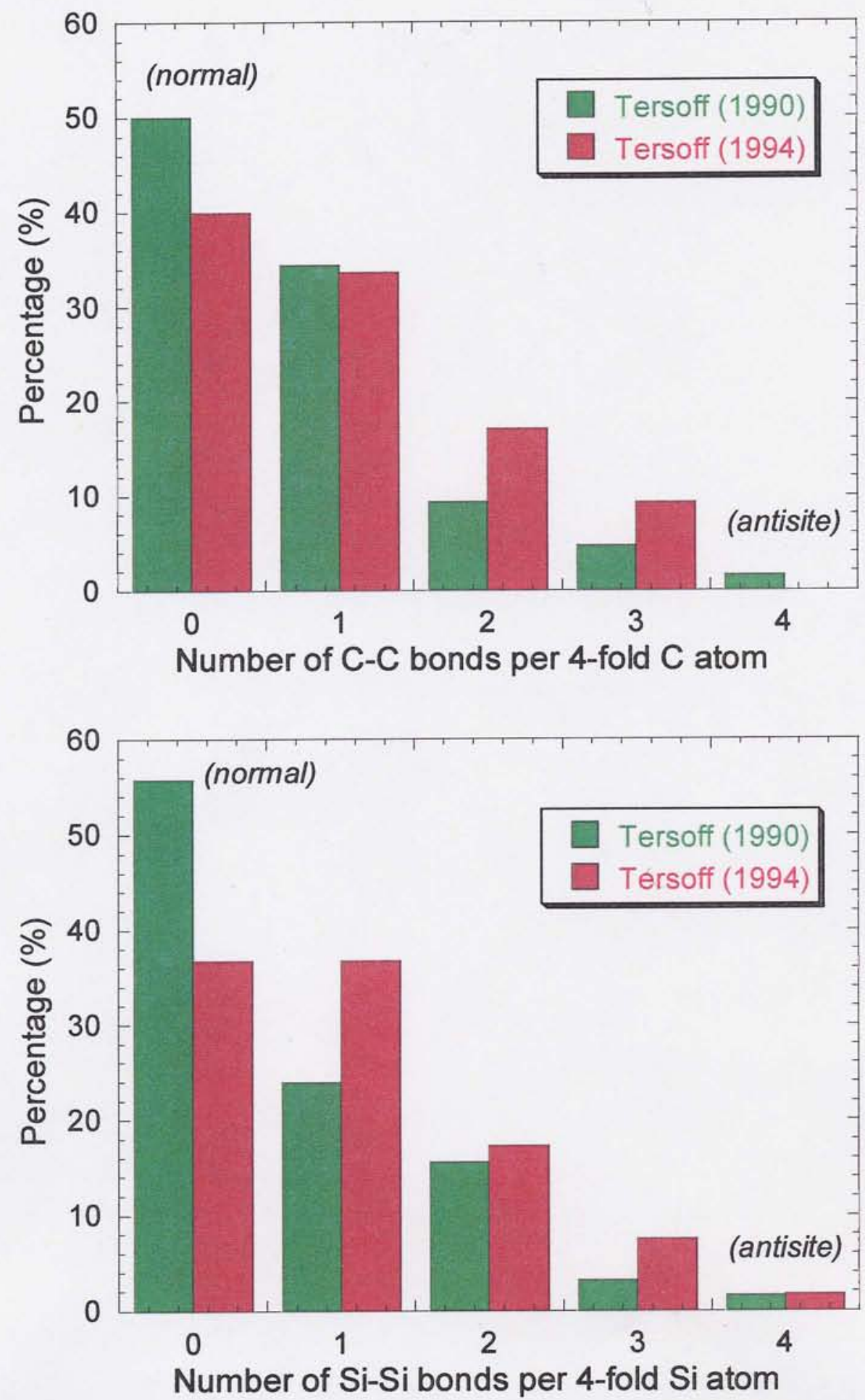

Fig. 19 Homonuclear bond distributions at $\mathrm{C}$ and $\mathrm{Si}$ sites for the final MDannealed structures of cascades embedded in $\beta-\mathrm{SiC}$, from Malerba and Perlado [123] using Tersoff 1990 potential and present work [117] using Tersoff 1994 potential. 
block of 216 unit cells with periodic boundary conditions imposed. Different initial antisite defect densities of $0.0 \%, 2.3 \%$. 5.8\%, 10.4\%, 16.4\%, 28.7\% and 50.0\% were established by swapping pairs of random sites. Target temperatures were adjusted by scaling the atomic velocities in the cell every five timesteps.

One set of the seven chemically disordered initial SiC configurations was equilibrated using MD at $300 \mathrm{~K}$ for $250 \mathrm{ps}$ (150,000 time steps) and data collected in the last $40 \mathrm{ps}$. A second identical set of seven was equilibrated at $2000 \mathrm{~K}$ for $500 \mathrm{ps}$, before cooling down to $300 \mathrm{~K}$ at $-10 \mathrm{~K} / \mathrm{ps}$ for $47 \mathrm{ps}$ and then equilibrating at $300 \mathrm{~K}$ for another $100 \mathrm{ps}$, the last $40 \mathrm{ps}$ of which were used for data collection. In order to study the bonding state of each atom, a cutoff-which was chosen as the first minimum of the total pair correlation function following the first coordination shell—was used to define bonds for that pair. The chosen cutoffs were $0.155 \mathrm{~nm}$ (C-C pairs), 0.193 (C-Si pairs) and 0.245 $\mathrm{nm}$ (Si-Si pairs).

The initial values of chemical disorder, $\chi_{0}$, corresponding to the engineered antisite densities for the seven chemically disordered $\beta$-SiC structures were 0.0 (no chemical disorder), $0.05,0.13,0.23,0.39,0.69$ and 1.0 (chemically random) respectively. Initially, these chemically disordered structures were in topologically perfect but highly unstable states, because the substitution of C-C $(0.155 \mathrm{~nm})$ or Si-Si $(0.245 \mathrm{~nm})$ bonds for C-Si (0.193) bonds, without changing bond lengths, introduced considerable instability. Annealing at $300 \mathrm{~K}$ or $2000 \mathrm{~K}$ was carried out to relax the configurations. As an example, a SiC structure with an initial value $\chi_{0}=1.0$ was generated by swapping 10,000 pairs of random sites. After equilibrating at $300 \mathrm{~K}$ for $200 \mathrm{ps}$, its configuration energy decreased $28 \%$ from -4.42 to $-5.64 \mathrm{eV} /$ atom, whereas $\chi$ remained unchanged at 1.0. Equilibrating instead at $2000 \mathrm{~K}$ for 500 ps decreased the configurational energy by $24 \%$ to $-5.48 \mathrm{eV} /$ atom and $\chi$ by $14 \%$ to 0.857 . Further cooling from $2000 \mathrm{~K}$ to $300 \mathrm{~K}$ at a rate of $-10 \mathrm{~K} / \mathrm{ps}$ reduced $\chi$ by another $1 \%$ to 0.848 and the configuration energy by a further $5 \%$ to $-5.73 \mathrm{eV} /$ atom. Evidently, most of the structural reconstruction occurred during annealing at $2000 \mathrm{~K}$. By way of reference, the fully equilibrated amorphous $\mathrm{SiC}$ modeled from initially random atom positions had a configuration energy of -5.73 $\mathrm{eV} /$ atom and $\chi=0.585$. The $2000 \mathrm{~K}$ equilibration temperature was chosen so as to be sure to mostly relax and not rearrange these disordered $\mathrm{SiC}$ structures; our modeling showed that the transition between these responses occurred at temperatures no lower than $2800 \mathrm{~K}$.

The distribution of homonuclear bonds on each site increased gradually as $\chi$ increased. Fig. 20 indicates the distribution of $\mathrm{C}-\mathrm{C}$ bonds at 4 -fold $\mathrm{Si}$ and $\mathrm{C}$ sites in $\mathrm{SiC}$ for different $\chi$; almost identical distributions were found for equilibration at $300 \mathrm{~K}$ and $2000 \mathrm{~K}$. The homonuclear bond distribution is controlled by probability, and thus by the bond ratio $\chi$. For small $\chi(<0.13)$, most antisites (atoms bonded entirely by homonuclear bonds) remained isolated, each contributing one atom with 4 homonuclear bonds and four neighboring atoms with 1 homonuclear bond each. Hence, sites with only 1 homonuclear bond are nearly four times more numerous than antisites when $\chi$ is small. As $\chi$ increases, there are more adjacent antisite defects resulting in partial antisites associated with 2 or 3 homonuclear bonds. For example, a pair of nearest neighbor antisites of opposite kind contributes a total of two atoms with 3 homonuclear bonds and six neighboring atoms with 1 homonuclear bond each. For completely chemically random $\operatorname{SiC}(\chi=1.0)$, adjacent antisites dominate, resulting in a broad homonuclear bond distribution. In no 


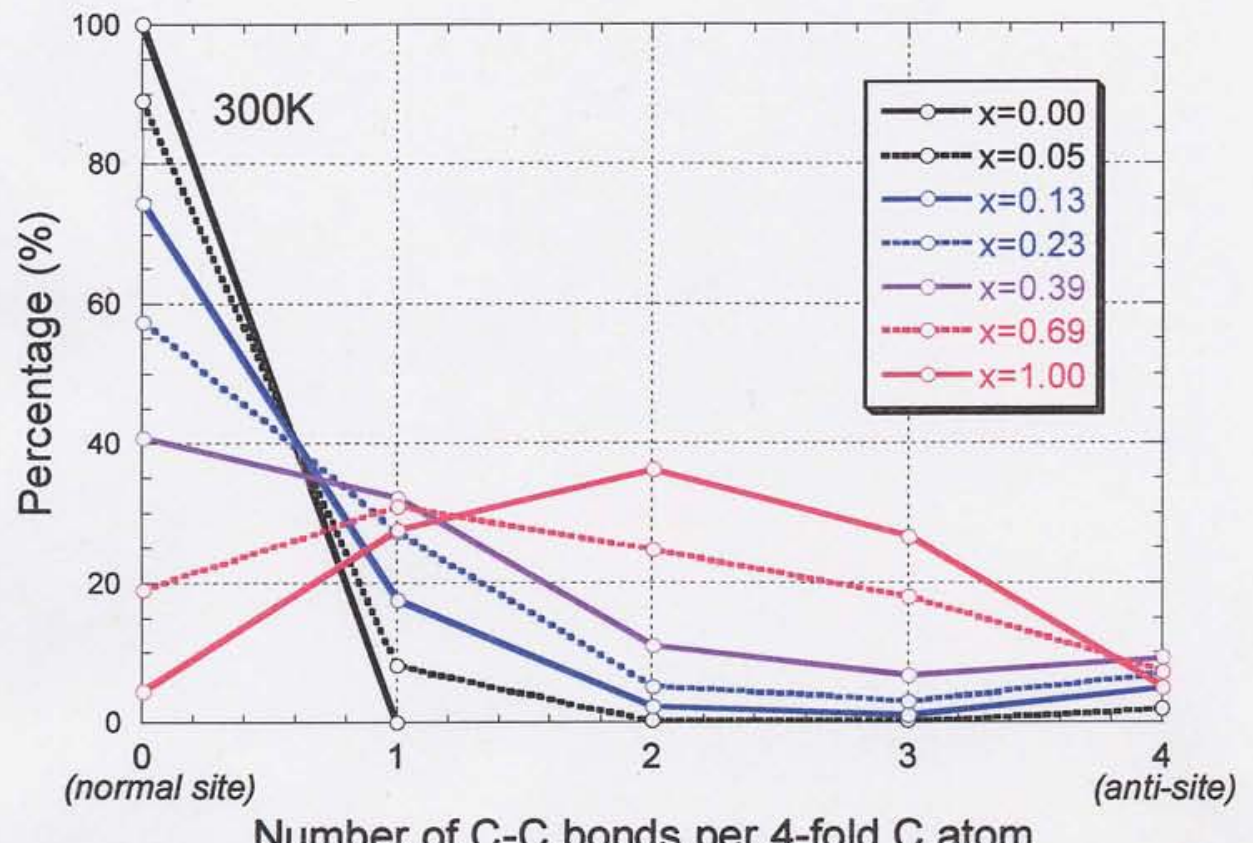

Number of $\mathrm{C}-\mathrm{C}$ bonds per 4-fold $\mathrm{C}$ atom

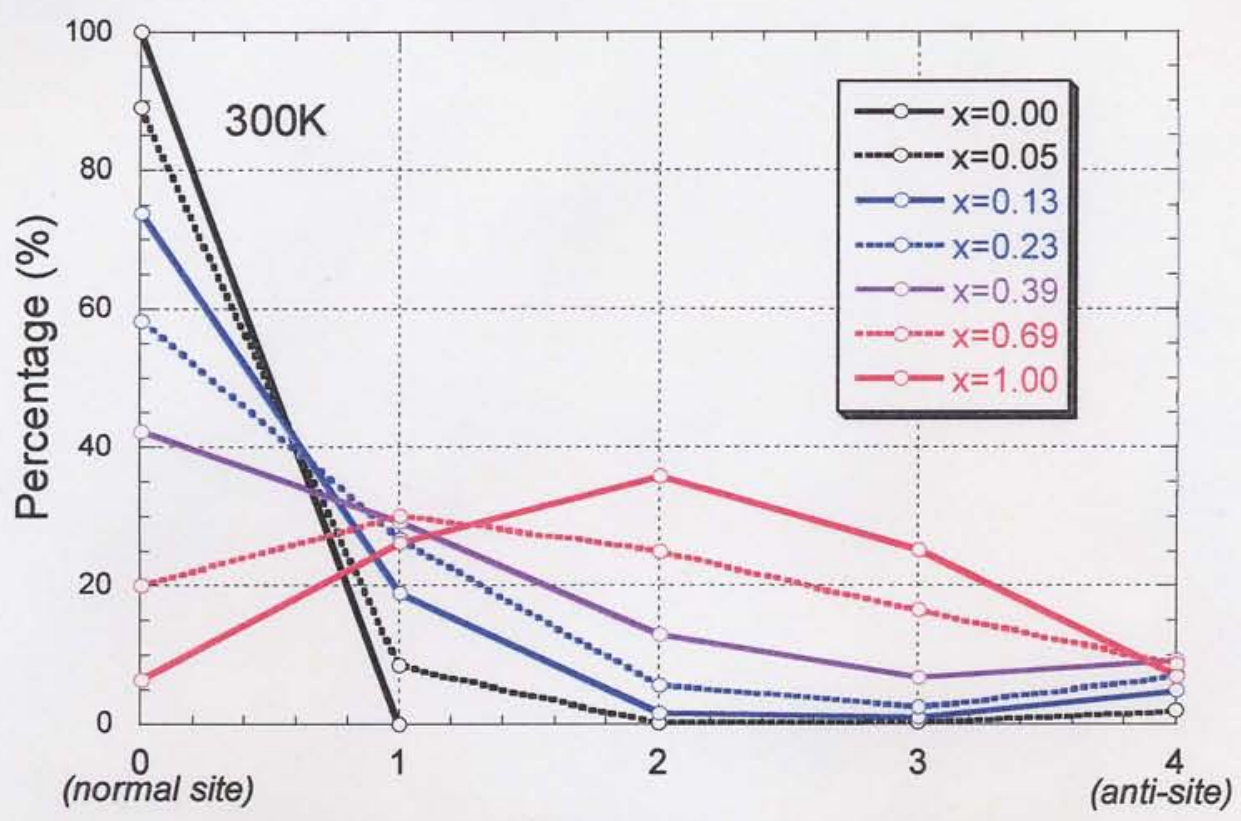

Number of Si-Si bonds per 4-fold Si atom

Fig. $20 \mathrm{C}-\mathrm{C}$ and $\mathrm{Si}-\mathrm{Si}$ homonuclear bond distributions for models of $\beta$-SiC, MDequilibrated at $300 \mathrm{~K}$, with increasing amount of chemical disorder $\chi$ introduced by initial random atom swapping [124]. Configurations with 2 and 3 carbon atom neighbors arise from close association of the resulting antisite defects. Lines were drawn between integer neighbor-count data points to better visualize trends. 
case were there ever more than $10 \%$ isolated antisites. Parallel analysis of 4 -fold Si sites was also carried out, with nearly identical results.

Fig. 21 depicts the changes in density and configuration energy with increasing $\chi$ at the two equlibration temperatures. The divergences at large $\chi$ result from the inability of the structure to undergo meaningful structural adjustment at temperatures as low as $300 \mathrm{~K}$. The initial fall in density and rise in energy result directly from the introduction of isolated antisite defects. Calculation from the initial energy-rise slope yields a formation energy of $7.5 \mathrm{eV}$ for an antisite pair $\left(\mathrm{C}_{\mathrm{Si}}+\mathrm{Si}_{\mathrm{C}}\right)$, compared to $7.2 \mathrm{eV}$ from an earlier calculation using the 1989 Tersoff potential [119] and 8.4-8.5 eV from an LDA $a b$ initio calculations [111,114]. Deviations from the initial slopes of either curve occur when antisite coordination shells start to overlap at associated antisites that require lower formation energy. Thus, small changes in $\chi$ disproportionately affect the density and configuration energy at small $\chi$.

By contrast, significant changes in topology occur only at larger $\chi$. Fig. 22 summarizes changes in Si coordination number and average local-cluster ring size during equilibration at $2000 \mathrm{~K}$. For $\chi \leq 0.39$, the local- and medium-range topologies of the initial $\beta$-SiC structure are maintained, with the majority of atoms being 4-coordinated and the local clusters having 12 6-rings. Significant topological alterations, in both Si CN and LC ring content, occur for the $\chi=0.63$ and 0.85 assemblies. The alterations in LCs at $\mathrm{C}$ sites and at $\mathrm{Si}$ sites were found to be identical, but the local configuration around $\mathrm{C}$ is somewhat more ordered than that around $\mathrm{Si}$, with $>97 \%$ of $\mathrm{C}$ sites in 4 -fold coordination. Fig. 22 therefore indicates that perfect topological order in the $\mathrm{SiC}$ structure is energetically stable until chemical disorder reaches beyond the $\chi=0.23-0.37$ range; for $\chi \geq 0.63$, topological perfection at $2000 \mathrm{~K}$ appears impossible to maintain. The effect of the $\chi$ topological order threshold is dramatically illustrated in Fig. 23 for initially topologically ordered $\beta$-SiC with two values of $\chi_{0}, 1.0$ and 0.23 , the first of which becomes topologically disordered at $2000 \mathrm{~K}$, the second remaining topologically ordered.

This topological result has profound implications for the thermophysical behavior of chemically disordered SiC. These were explored by heating the assemblies equilibrated at $100 \mathrm{~K}$ continuously to $7000 \mathrm{~K}$ at a rate of $10 \mathrm{~K} / \mathrm{ps}$ and evaluating the simulation cell volume and configurational energy. The cell volume results for assemblies with initial values $\chi_{0}=0.0 .0 .23,0.39$ and 1.0 are shown in Fig. 24a. A similar plot was obtained for the configurational energy, Fig. 24b. The heating curves show that for $\chi_{0} \leq 0.39$, the configuration undergoes a distinguishably sharp melting transition. For $\chi_{0}>0.63$ (for example, at $\chi_{0}=1$ illustrated), the assembly instead exhibits a continuous glass transition, which is nearly reversible on cooling. Annealing the structure with $\chi_{0}=1.0$ at $2000 \mathrm{~K}$ resulted in a substantially, but not wholly amorphized configuration (with $\chi=0.85$ ) that underwent a further transition at $3000 \mathrm{~K}$ (near the glass transition) to more complete topological disorder (with $\chi=0.62$ ). The evolution of this network configuration is shown in Fig. 25, in which the amorphizing transition (by 2000 $\mathrm{K}$ ) and the subsequent glass transition (at $3000 \mathrm{~K}$ ) are clearly evident. Fig. 26 tracks the corresponding changes in configurational energy, chemical disorder and 6-ring topology through the glass transition occurring at $3000 \mathrm{~K}$. 

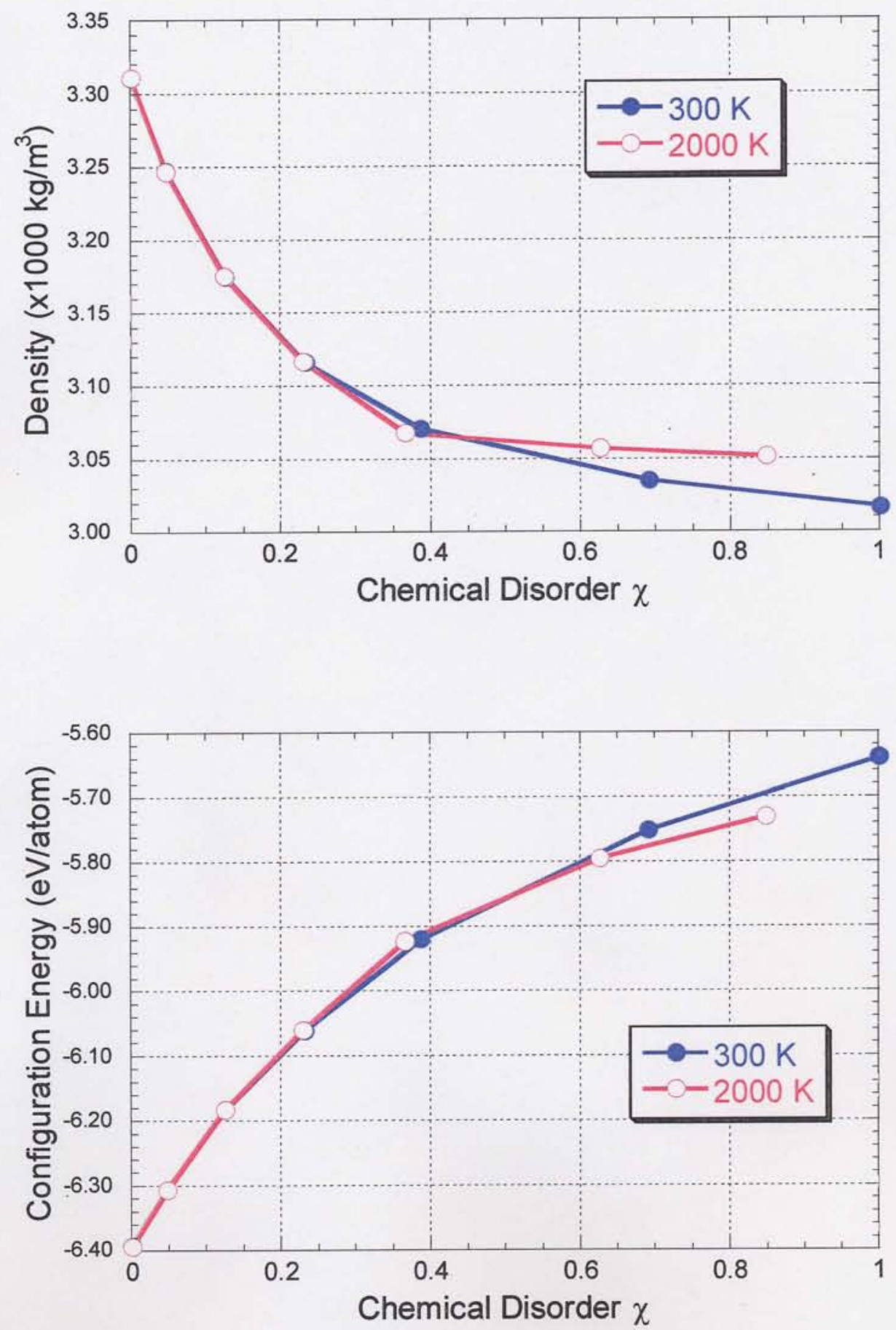

Fig. 21 Effect of chemical disorder $\chi$ on a) density and b) configuration energy of $\beta$-SiC simulation cell, MD-equilibrated at $300 \mathrm{~K}$ and $2000 \mathrm{~K}$. 

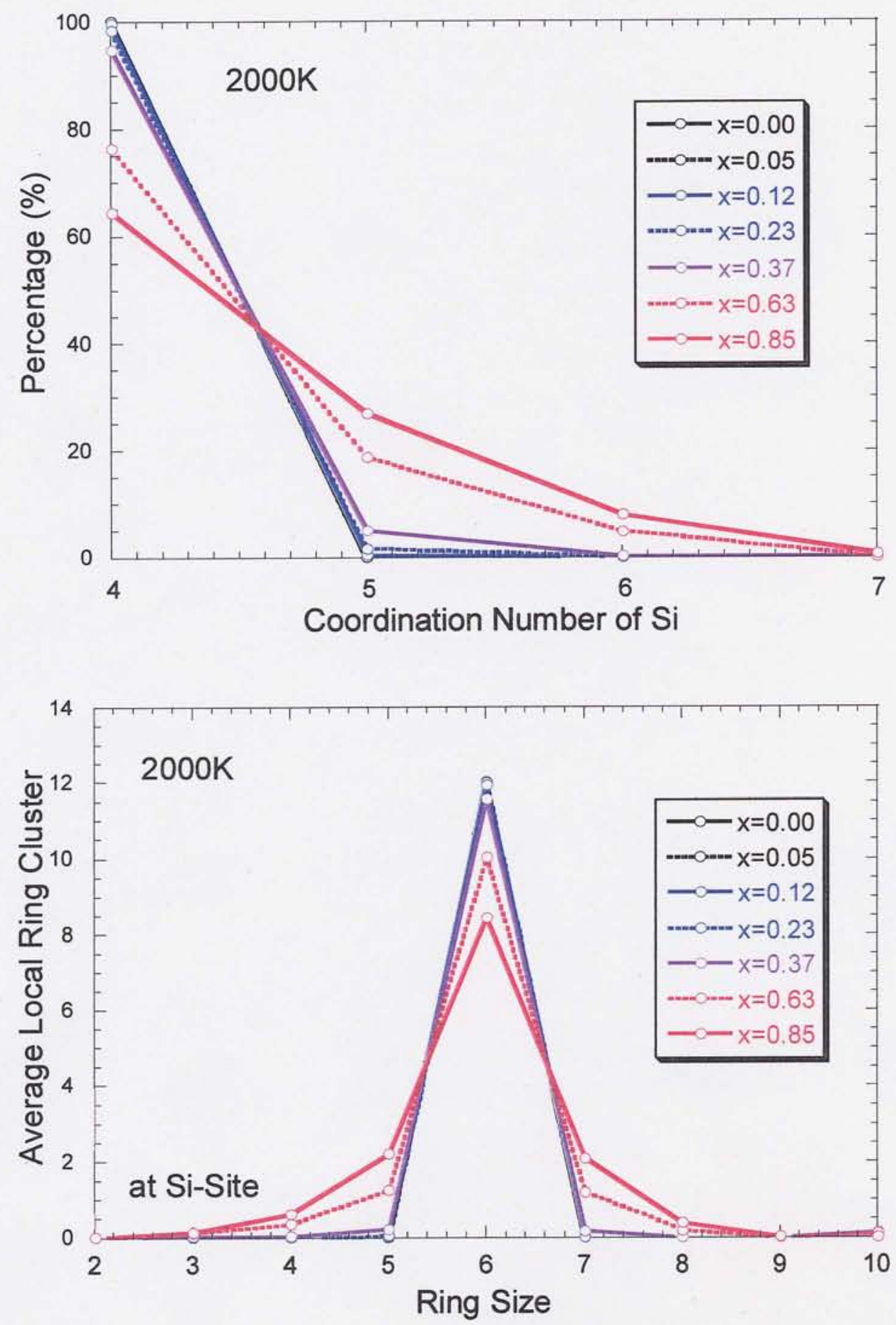

Fig. 22 Distributions of a) Si coordination and b) local cluster ring size at Si sites for $\beta$-SiC simulation cell, MD equilibrated at $2000 \mathrm{~K}$, as a function of resulting chemical disorder $\chi$. Lines are drawn between integer $\mathrm{CN}$ and ring count data points to better visualize trends. 


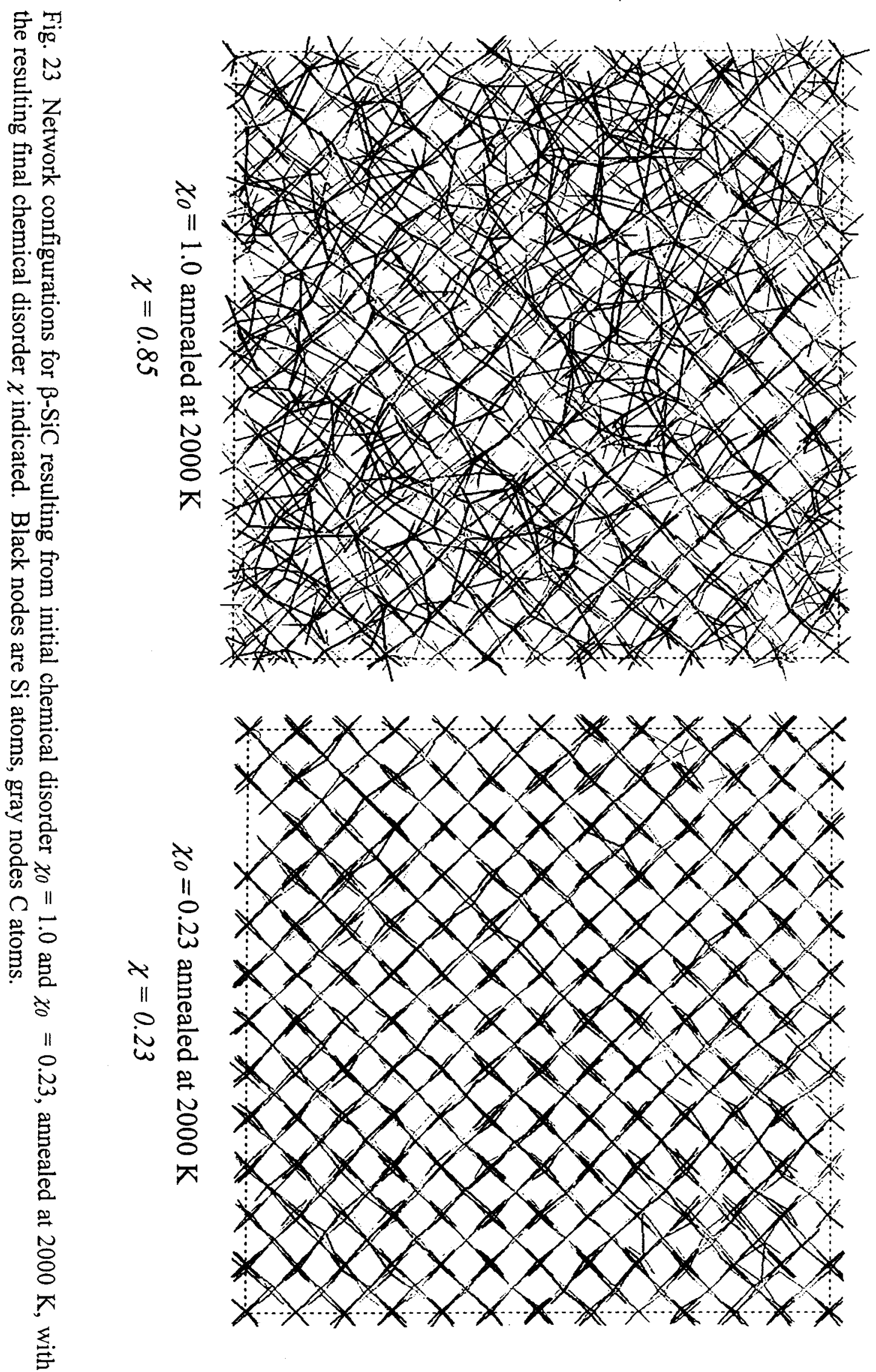



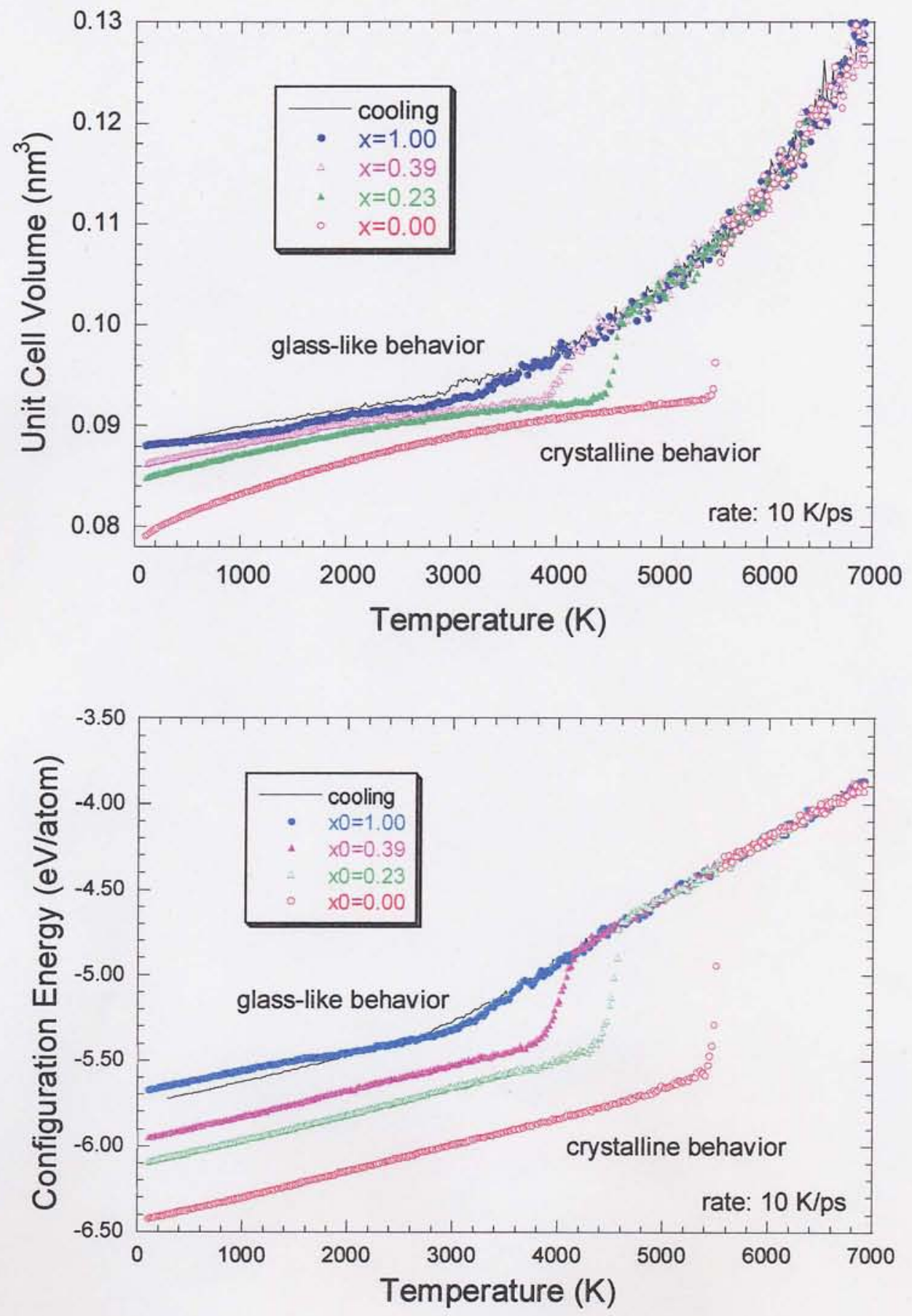

Fig. 24 Behavior of a) cell volume and b) configuration energy during heating and cooling (at $10 \mathrm{~K} / \mathrm{ps}$ ) of chemically disordered $\beta$-SiC, originally MDequilibrated at $100 \mathrm{~K}$. Assemblies with large initially imposed chemical disorder $\chi_{0}$ underwent a reversible glass transition, those with lower $\chi_{0}$ a melting transition. 

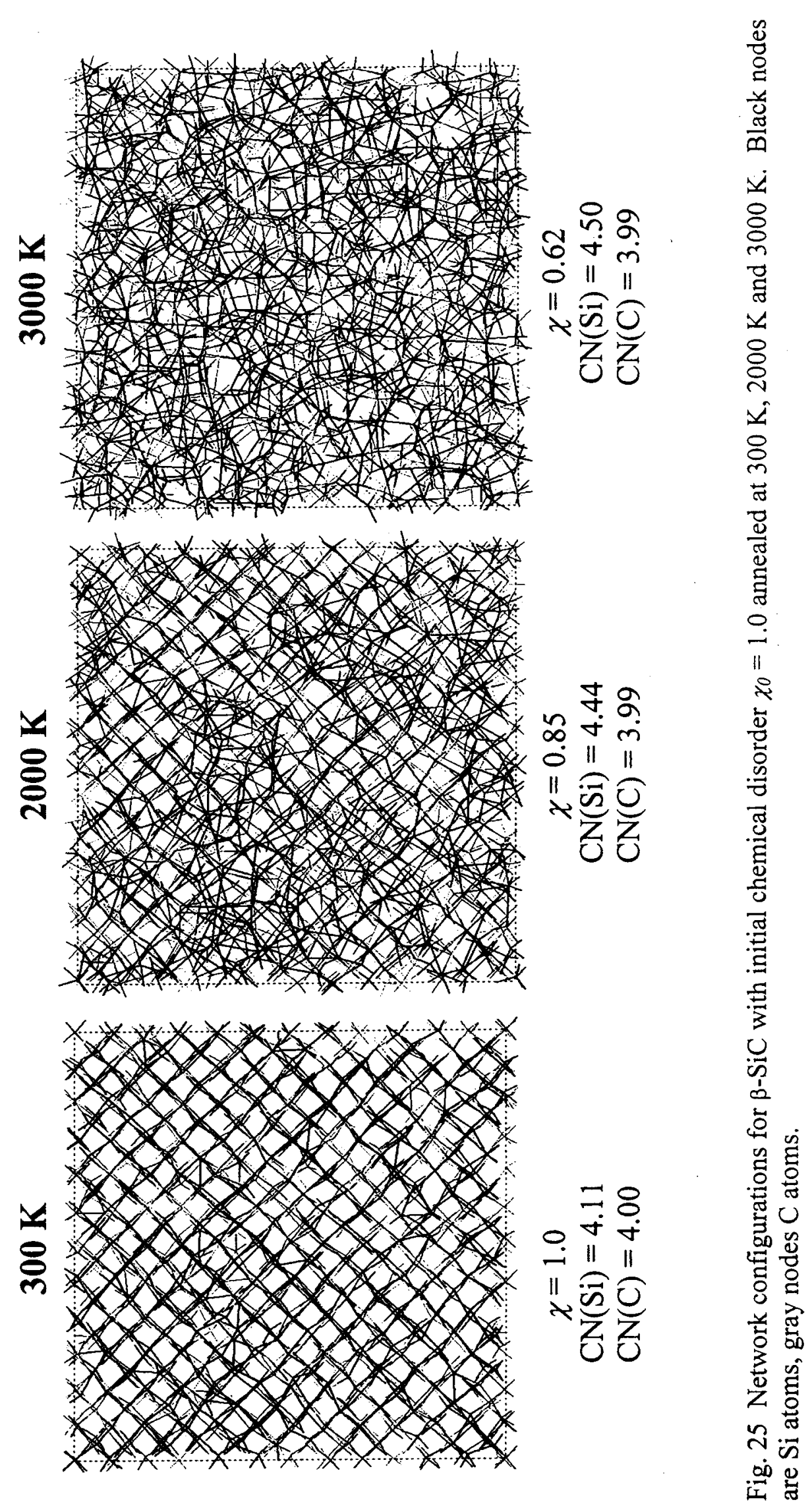


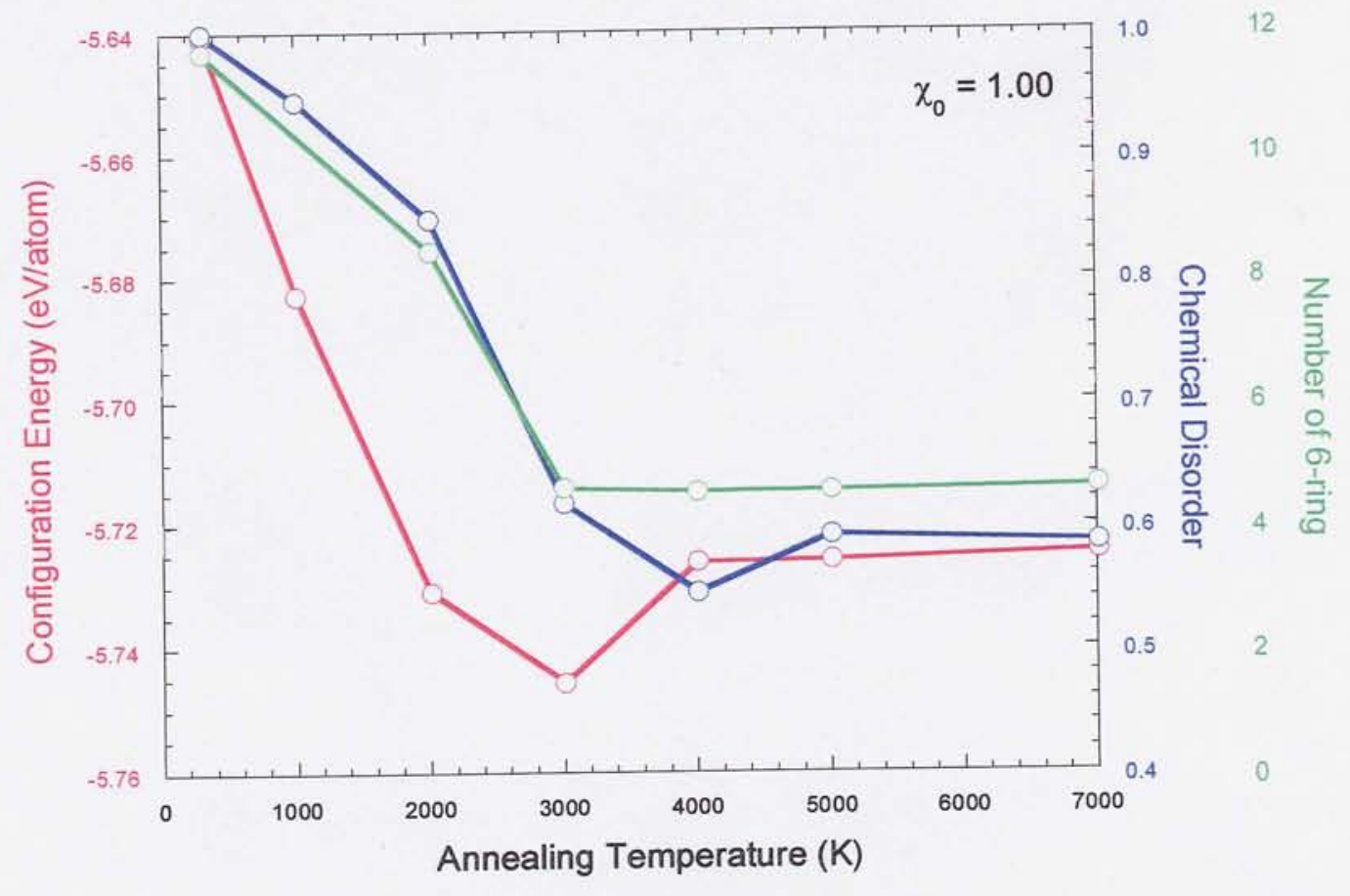

Fig. 26 Changes in configuration energy, chemical disorder and 6-ring topology in fully chemically-disordered $\beta$-SiC through topological disordering (by $2000 \mathrm{~K}$ ) and through the glass transition at $3000 \mathrm{~K}$. 


\subsection{The Amorphizing Transition in SiC}

The MD simulations described in $\S 4.2$, coupled to the topology and rigidity considerations developed in $\$ 4.1$, have enabled a fundamental understanding of the role that chemical disorder plays in the amorphization of SiC. The ability to form homonuclear bonds is what distinguishes $\mathrm{SiC}$ from $\mathrm{Si}_{3} \mathrm{~N}_{4}$ and $\mathrm{SiO}_{2}$ structures and makes possible antisite defect formation. The possibility of forming homonuclear bonds in $\mathrm{SiC}$ removes coordination constraints that would otherwise render crystalline $\mathrm{SiC}$ topologically very difficult to amorphize. Because formation of homonuclear bonds is (at least initially) a necessary consequence of antisite formation and likely outcome of Frenkel interstitial accommodation, both defect types doubtless contribute to the amorphizing transition. Homonuclear dumb-bell interstitials are indeed found to be the dominant interstitital configuration in displacement simulations [107]. Excluding homonuclear bonds would preclude antisite defect formation and render Frenkel defects far less stable and less likely to integrate into the amorphizing network; it would also make amorphization impossible. We believe we have demonstrated that homonuclear bond formation plays a key role in $\mathrm{SiC}$ amorphization.

The behaviors depicted in Figs. 22-24 suggest strongly that there is a chemical disorder threshold in the vicinity of $\chi=0.3-0.4$ for amorphization of SiC, and that topological disorder is strongly preferred for $\chi>0.5$. This conclusion is in keeping with structural freedom arguments and supports our earlier topological modeling observation [105] that topological disordering of $\mathrm{SiC}$ without chemical disorder $(\chi=0)$, i.e. keeping [SiC $\left.{ }_{4}\right]$ coordination tetrahedra intact) is impossible to achieve. It is also consistent with analyses of an $a b$ initio MD-simulated amorphous SiC [114] and of Tersoff's a-SiC assembly modeled with a smooth cutoff [113], which give $\chi=0.6$ and 0.5 respectively. Recently completed MD simulations, carried out by Professor Yip's group [125] in parallel with the topological MD results reported here, have corroborated the role of a critical local homonuclear bond density in effecting amorphization. In these simulations, an 8000-particle $\beta$-SiC supercell, into which Frenkel and anitsite defect distributions consistent with collision cascade simulations were embedded, was equilibrated using the MDCASK code developed at Lawrence Livermore National Laboratory [126]. A critical level of homonuclear bonds was found necessary to drive heterogeneous nucleation of amorphous pockets in addition to interstitial defects.

While major topological changes are possible at large $\chi$, including amorphization and a glass transition, small changes in $\chi$ at small $\chi$ contribute disproportionately to changes in density and configurational energy and result in only trivial changes in topology. Almost $80 \%$ of the density change on amorphization can be attributed to accumulation of chemical disorder alone below the $\chi$ threshold, providing an explanation for the anomalous low-temperature "point defect swelling" of irradiated SiC, noted long ago [127].

\section{ON-GOING RESEARCH INTO AMORPHIZED STRUCTURES}

Several areas of current research are still in progress, and have not yet generated the definitive level of results outlined in $\S \S 2-4$, but are described below. In many cases, 
our thinking about the problem has been informed by our earlier conclusions, and the discussion below reflects our current thinking about the approaches we have adopted for continuing work in these areas.

\subsection{Characterization of Topological Disorder by Electron Diffraction and Imaging}

Characterization of intermediate-range structure in amorphous materials has long been a difficult task for experimental approaches owing to a lack of suitably sensitive characterization tools. MD simulations provide explicit atom positions and local ring topologies, but in the absence of periodicity atom positions cannot be obtained through experimental study and even ring statistics cannot be experimentally verified. Our recent study shows that dominant ring topologies are evidently associated with the first sharp diffraction peak [88], but not in a straightforward way. Recently, a real-space imaging method has been proposed as a way of studying of intermediate-range order in amorphous network semiconductors $[128,129]$. The method records image intensity fluctuations across dark-field TEM images of variable coherence that can be related to correlations of higher order than simple pair correlations; such higher order correlations can be compared to those obtained from simulations, or the simulations can be used to generate flutuation maps for comparison.

\subsubsection{First Peak in Electron Diffraction}

Fig. 13 illustrates data from energy-filtered electron diffraction (EFED) measurements of amorphized silicas obtained on the Vacuum Generators HB5 100-kV predecessor of our current HB603 300-keV field-emission STEM out to Q $100 \mathrm{~nm}^{-1}$. Fig. 27 demonstrates (with a polycrystalline gold calibration standard) the improved sensitivity and accuracy of the latter current instrument in this mode out to $Q>150 \mathrm{~nm}^{-1}$ ( $\mathrm{q}=\mathrm{Q} / 2 \pi=25 \mathrm{~nm}^{-1}$ ). On the other hand, our simulations of neutron diffraction (ND) spectra from silica assemblies of widely differing topologies, and the pair-correlations derivable from them, Fig. 12, show that much of the extended diffraction spectrum provides little additional information about topological differences. Neutron diffraction data, in fact, represent the best-case scenario, because atom scattering factors for thermal neutrons remain constant with scattering angle, whereas electron scattering intensities fall

roughly as $\mathrm{q}^{-4}$. Even so, only the first sharp diffraction peak provides adequate diagnostic shifts to be useful, as seen in Fig. 14.

These two experimental facts together mean that there is little point in pursuing electron diffraction data to higher $Q$, because little additional information about topological differences could be obtained, even if the data were rendered statistically reliable. Instead, our focus will be on characterizing with as much clarity and precision the position and shape of the first sharp diffraction peak in electron diffraction. This is, from an intensity point of view, the most accessible information in any event for electron diffraction. Fig. 28 shows data taken with our HB603 STEM for four $\alpha$-SiC TEM samples, ion-amorphized by Dr. Lu-Min Wang of the University of Michigan in the Argonne HVEM-Tandem Ion Accelerator facility. The first diffraction peak is, of course, prominent for each, and differences in the peak position and shape are well resolved. 

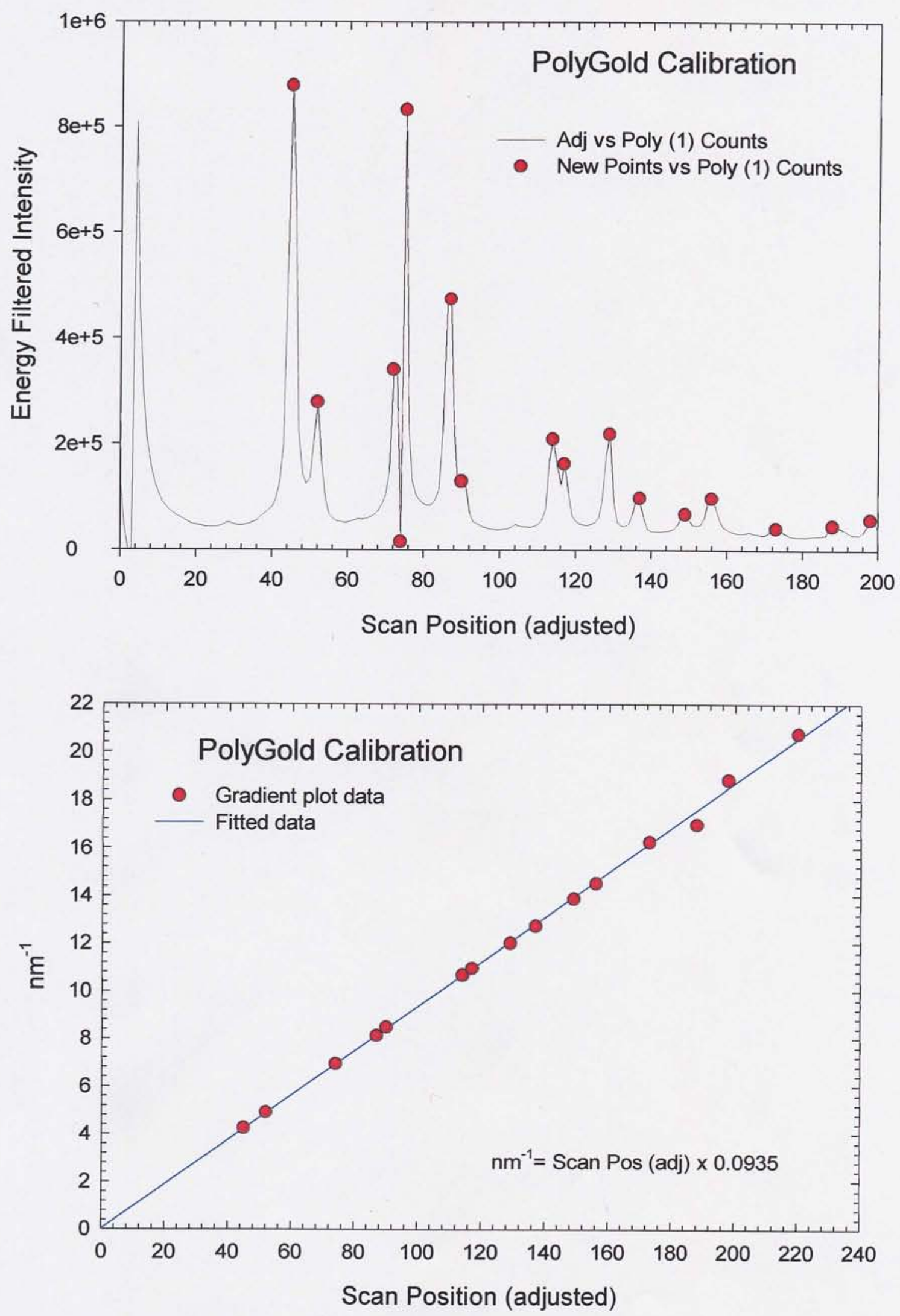

Fig. 27 Experimental electron diffraction data and calibration to $q(=Q / 2 \pi)$ (polycrystalline gold standard) using VG HB 603 STEM and serial EELS. 


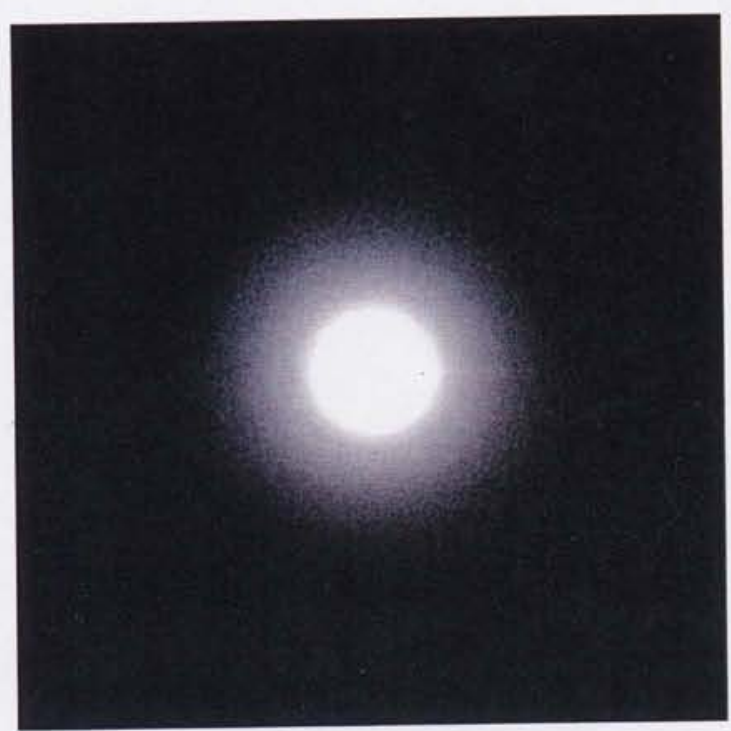

(a)

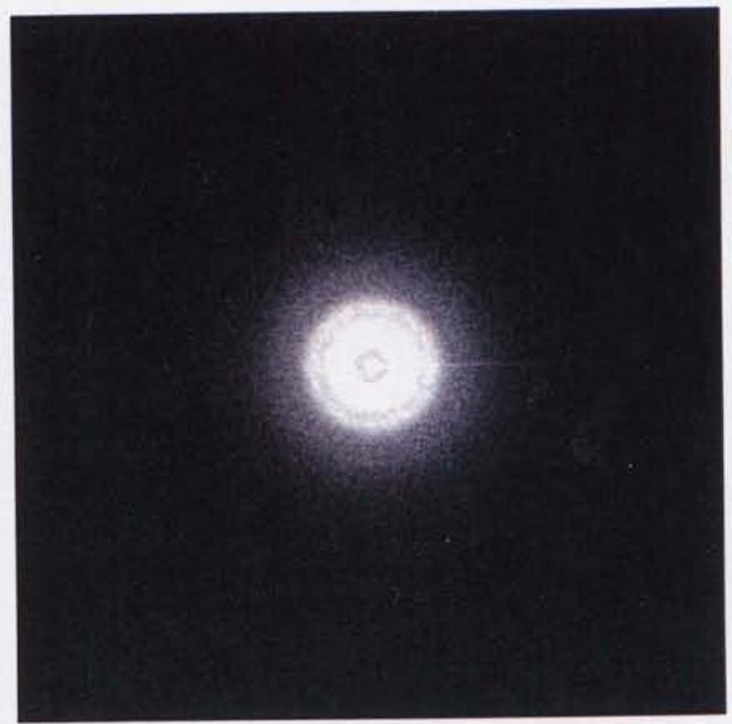

(b)

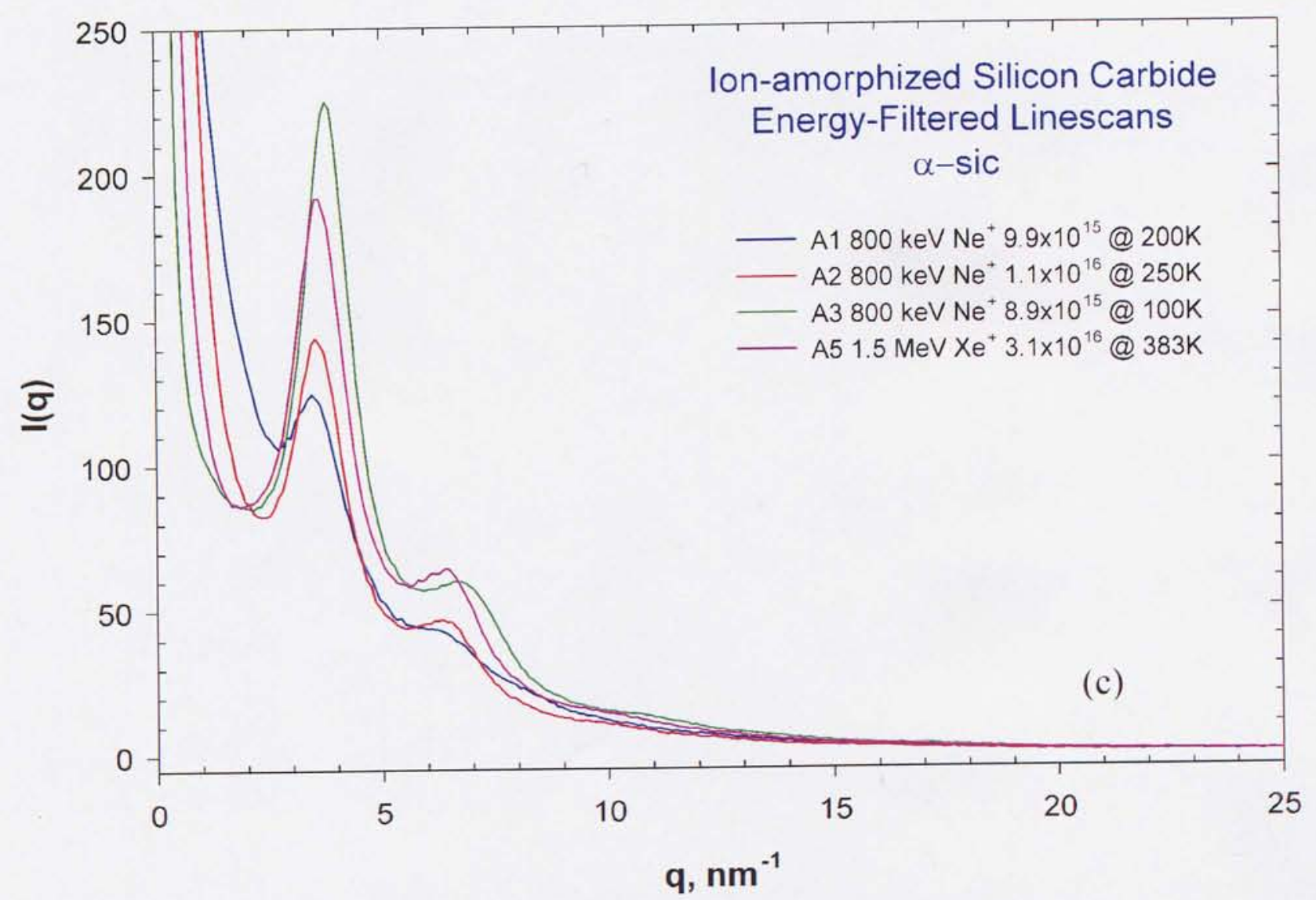

1. In: r thin fril

Fig. 28 Electron diffraction patterns collected in HB603 STEM from ionamorphized $\alpha$-SiC thin foil: a) unfiltered; b) energy-filtered with $5 \mathrm{eV}$ window centered on zero loss. c) EFED dedicated linescans yielding $I(q)$ for four samples diffraction peak. 
The theory for information contained in the first diffraction peak is not well worked out yet [96], but with our MD models with designated topologies we now have a way to correlate information appearing at the first peak with known topological features. As well as continuing to acquire experimental EFED data from $\mathrm{SiO}_{2}, \mathrm{SiC}, \mathrm{Si}_{3} \mathrm{~N}_{4}$ and $\mathrm{Si}$ amorphized networks, we are pursuing this correlation by comparison of MD-synthesized topological models of these networks, well characterized in their intermediate-range topologies, with their corresponding calculated electron diffraction intensities simulated in the region of the first peak. These comparisons should eventually provide a powerful data base from which to interpret experimental EFED first-peak details.

\subsubsection{Fluctuation Microscopy of Amorphized $\mathrm{SiO}_{2}, \mathrm{SiC}$ and $\mathrm{Si}_{3} \mathrm{~N}_{4}$}

As indicated above, diffraction generally provides structural information in the form of atom pair correlations that, while sensitive to short-range order, are substantially less sensitive to intermediate-range structure. Direct imaging potentially offers additional information on atomic structure over that supplied by area-averaged diffraction, but conventional high-resolution TEM images of amorphous solids do not provide even lateral atomic-level structural information, because projection through the specimen thickness does not present an invariant projection, as in crystals oriented along prominent symmetry axes [130]. In most cases, the amorphous material serves as little more than a test object for randomly sampling the imaging lens transfer function, though non-random features can sometimes be extracted using auto-correlation or information theory methods [131].

Higher order (third or fourth order) correlations (for example, pair-pair correlations) have recently been shown to be much more sensitive to intermediate-range structure [128], and a microscopy-based method has been recently explored (variously dubbed variable coherency microscopy [128] or fluctuation microscopy [129]) which is sensitive to intermediate-range structure in the presence of disorder. The higher order correlations are revealed from the fluctuations of the intensity diffracted from sample volumes, over which illumination coherence is maintained, that have the scale of intermediate-range structure components. The practice involves varying the lateral coherence of the electron illumination by varying the inner cone angle in hollow-cone illumination in conventional TEM or alternatively the effective width of the annular darkfield aperture in STEM [132], then measuring the variance (speckle) of intensity in the dark-field images at low resolution. The fluctuation variance $V(Q)$ is defined as

$$
V(\boldsymbol{Q}, \kappa)=\left\langle I^{2}(\boldsymbol{Q}, \kappa)>/<I(\boldsymbol{Q}, \kappa)>^{2}-1\right.
$$

where $<>$ indicates averaging over images, $I(\boldsymbol{Q}, \kappa)$ is the image intensity, $\boldsymbol{Q}$ is the scattering vector, and $\kappa$ is a measure of image resolution related to the reciprocal-space radius of the objective aperture in CTEM or probe radius in STEM. For the isotropic amorphous samples, $I(Q)$ is typically averaged azimuthally. Variable coherence microscopy [128] amounts to measuring $V(Q)$ at constant $\kappa$, for which a deliberately low image resolution of $\sim 1.5 \mathrm{~nm}$ is used. Information comes from the position and height of peaks in $V(Q)$ vs. $Q$ curves. Peaks are generally found at the positions of diffraction

maxima of the underlying materials. The height of these peaks indicates the relative 
intermediate-range correlation strengths in the material. Fluctuation microscopy [129] systematically maps out the full locus of $V(Q, \kappa)$ as a function of changes in both parameters.

Hollow-cone dark-field images are built up from many coherent tilted dark-field (TDF) images. For MD simulated structures, the TDF images can be computed using the phase-grating approximation [133]. The situation approximates a sample with a transmission function that is the potential of the sample projected along the direction of travel of the electron beam. The TDF image intensity is the squared modulus of the incident wave multiplied by the transmission function and then convolved with the microscope point-spread function. To compute the intensity for an incoherently filled hollow-cone annulus of finite width, the TDF image intensities are summed for many incoming wave vectors inside the annulus.

We are pursuing this technique, using our VG HB603 UHV 300-kV fieldemission STEM, instead of the conventional TEM fitted with hollow-cone illumination employed by Gibson, Treacy and Voyles [128,129]. There are two ways in which the STEM can be configured to provide the desired information. In the first, the annular dark-field (ADF) mode, a somewhat defocused (diameter $\kappa$ ) electron probe of small convergence angle $(\sim 1 \mathrm{mrad})$ is scanned across the specimen and an image formed serially from scattered electrons collected on a narrow annulus of the dark-field detector; by reciprocity, this configuration is optically conjugate to hollow-cone illumination in TEM. Altering the probe diameter (by defocusing the probe) alters $\kappa$ directly, instead of relying on the extent of lateral coherence to define indirectly the sample volume across which correlation-enhanced diffracted intensity emanates. The dark-field detector field is easily masked to a narrow ring, and the HB603 post-specimen lenses used to select the band in $Q$-space from which to form speckle images.

Alternatively, and more powerfully, we use the STEM in a nanodiffraction mode, again with a variable probe diameter (greater than the correlation lengths to be probed), and collect the whole diffraction pattern $I(Q)$ in parallel, over all $Q$ of interest simultaneously, from each volume of the sample defined by the probe area and addressed in turn. The probe scan coils are easily programmed to step areas sequentially. This method makes far more efficient use of incident electrons, since information over all $Q$ of interest is collected in parallel. Nanodiffraction patterns are collected using an existing fluorescent screen in the HB603, optically coupled to an available $1024 \times 1024$-element slow-scan CCD detector with cooled sensor and frame store; again, the post-specimen lenses are used, this time to scale the diffraction pattern to the size of the CCD. $I(Q)$ in each nanodiffractogram is then simply azimuthally averaged at each selected $Q$ increment and statistically totaled for all the areas addressed to generate $V(Q)$ at constant $\kappa$. Altering the probe size generates another $V(Q)$ series at a second $\kappa$, and so forth to generate $V(Q, \kappa)$.

In both configurations, we have discovered the need to establish probe sizes rather larger and probe convergences rather smaller than usual for an imaging STEM probe. This is best accomplished by reducing the excitation of the objective lens (to achieve focal lengths $\sim 10 \mathrm{~mm}$ ) and defining the probe size and convergence with a small real objective aperture-for which a multiple holder exists in our HB603 columninstead of with the virtual objective aperture (VOA). A set of 5, 10, 15 and $20 \mu \mathrm{m}$ objective apertures under these conditions yield probe diameters of 6, 3, 2.3 and $1.5 \mathrm{~nm}$ 


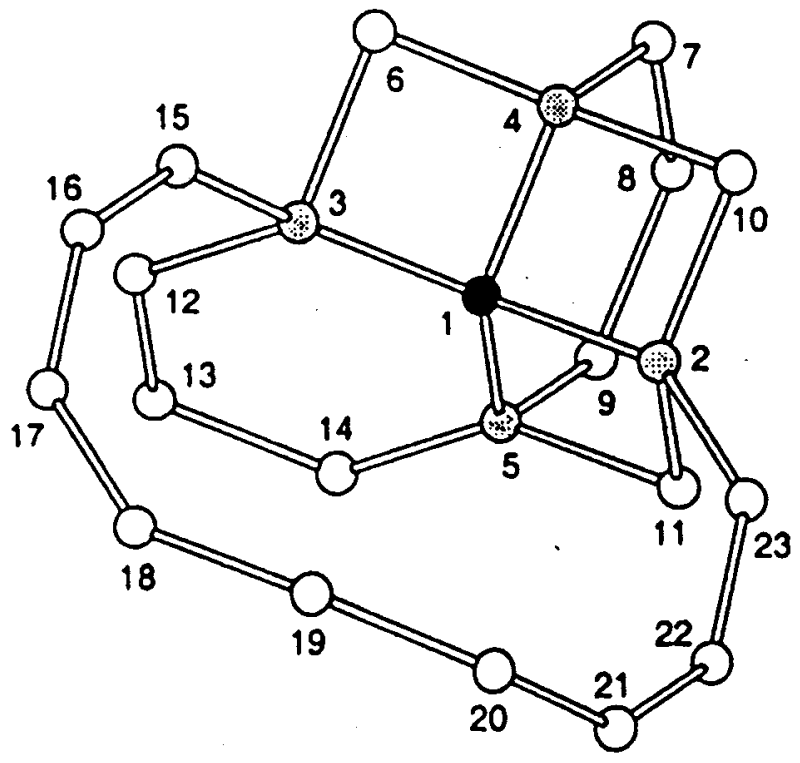

Fig. 29 The local cluster of the zeolite faujasite (O'Keeffe form of the Schläfli cluster) reproduced from Fig. Ic of Treacy et al. [151]. 
(further adjustable by changing objective lens excitation) with acceptable convergence angles between 0.25 and 1 mrad. Poor electron statistics in this alternative arrangement prove an issue with the large probes and indirect detection through the fluoresecent screen and CCD, addressable by longer counting times which demand better mechanical stability and higher emission stability. Both short- and long-term emission stability are, in fact, recognized problems encountered with cold field emitters, such as used in the HB603. The residual current intercepted by the (now redundant) VOA can, however, be easily used to monitor variations in the emission current continuously and so correct measured $I(Q, \kappa)$ data. Because fluctuation microscopy is effectively a high-resolution technique, the usual formation of an (also amorphous) hydrocarbon contamination layer under the intense electron probe is a serious problem that seriously affects measured correlations. The UHV STEM, which itself introduces almost no contamination, is certainly a better instrument from this standpoint than a non-UHV TEM/STEM instrument for the nanodiffraction configuration and probably also better than a conventional TEM in hollow-cone illumination mode. A plasma cleaner is installed on our HB603 to remove any incidental contamination brought in with the specimen. Because fluctuation microscopy is still essentially a high-resolution microscopy technique we cannot average over large areas to minimize the electron beam exposure during analysis, as we can in EFED. Hence, it is certain that the atom arrangements continue to change significantly during image recording, and we can expect that the data may be seriously flawed. The nanodiffraction configuration in STEM has the advantage of making much more efficient use of diffracted electrons than do the dark-field TEM or ADF STEM geometries, which means that data acquisition times are shorter, with a corresponding reduction in ionizing radiation load to specimens, like $\mathrm{SiO}_{2}$, sensitive to radiolytic displacements. Of large potential interest to us is the comparison of EFED measurements of the first diffraction peak with the fluctuation microscopy assessment of intermediate-range structure.

\subsection{Further MD Simulation of SiC Amorphization}

It has been shown in our previous study described in $\S 4$ that chemical disorder plays an important role in $\mathrm{SiC}$ amorphization, and the thermophysical behavior of $\beta$-SiC crystal changes gradually from crystalline behavior to glass-like behavior as the initial chemical disorder ratio $\chi$ increases. Our further study is focused on how chemical disorder and topological disorder change within and above the transition range. MD annealing of $\mathrm{SiC}$ structures with various imposed chemical disorder ratios is conducted at different temperatures around their transition ranges. These annealed structures are then cooled down to room temperature and analyzed.

\subsubsection{Ab-initio MD Modeling of Silicon Carbide Structure}

5.2.1.1 Shortcomings of topological modeling. One problem of using the topological modeling approach to study a-SiC structures is that many Si sites are not tetrahedral. Our MD study using Tersoff 1989 and $1994 \mathrm{SiC}$ potentials shows that there

are only about 30\% 4-coordinated Si sites in a-SiC. Even with the 1990 Tersoff potential, there are still over $20 \%$ Si sites that are either 3- or 5-coordinated. Earlier $a b$ 
initio MD (AIMD) simulations [114] also suggest that there are less than 70\% 4-fold Si sites in a-SiC. Despite the great variation in numbers for the different approaches, it is clear that a significant portion of Si sites are not in tetrahedral configurations. While 5fold $\mathrm{Si}$ is hard to model with our regular topological modeling method, 3-fold Si has quite different geometry (not triangular like $\left[\mathrm{BO}_{3}\right]$ polytopes) and is not simply an underconnected 4-fold Si atom. Therefore, topological modeling based on Si/C-tetrahedral building blocks alone is not completely suitable for study of a-SiC local structures without additional polytopic modifications.

5.2.1.2 Shortcomings of $\mathrm{MD}$ simulation. MD studies of $\mathrm{SiC}$ cascades have now been conducted by several groups. One of the most crucial parameters for MD simulation is interatomic potential. It has been shown that any of the Tersoff empirical potential variants is more appropriate for $\mathrm{SiC}$ modeling than are the Pearson [121] or modified EAM[122] potentials, and recent SiC simulations have mostly used the Tersoff potential forms. However, the Tersoff potential comes in several versions, which produce many contradictory results, most notable significantly different local topologies. For example, an almost topologically perfect structure can be obtained from embedded aSiC with the 1990 Tersoff potential, whereas such perfect structure is nearly impossible to obtain with other Tersoff potentials, due to a large fraction of non-tetrahedral Si sites that impede the retention of topologically perfect structures.

As found in the case of MD study of boron glasses, as well as of a-SiC in this study, it is very difficult to use MD simulation techniques using empirical potentials to model structures with species that frequently change their configurations. Compared to earlier AIMD study of a-SiC [114], MD simulation with any version of Tersoff empirical potentials yields significant differences in $\mathrm{C} / \mathrm{Si}$ local configurations. For example, our MD simulations of a-SiC with the various Tersoff potentials [117] generated 26\% (1989), 80\% (1990), and 33\% (1994) tetrahedral Si sites, whereas AIMD gives 65-70\% tetrahedral Si sites [114]. In addition, Si-Si pair radial distribution functions and homonuclear bond ratios differ in empirical-potential (classic) MD and AIMD simulations. Discrepancies in the formation enthalpies and stability of various defect configurations (antisites, interstitials) are also seen [111]. Obviously, these differences have the potential to lead to conflicting conclusions, as shown in our recent study. As a result, many details about a-SiC remain unconfirmed and will profit from AIMD analysis.

5.2.1.3 Ab-initio molecular dynamics (AIMD) simulation. The biggest problem with AIMD is its size limitation. Instead of the thousands or even millions of atoms required in knock-on cascade simulations, AIMD simulations usually number at most only hundreds of atoms. In our topological studies, however, simulation size is seen as less significant than the accurate description of Si and $\mathrm{C}$ local structures. A previous study of silicate systems [48] suggests that the size influence on topology can be neglected once the system size is much larger than the largest primitive rings in the system. The largest primitive ring in the classic MD-simulated a-SiC is about a 10-ring, which translates to a minimal diameter of about $1 \mathrm{~nm}$, or 100 atoms. Furthermore, the $\mathrm{SiC}$ system should be less influenced by size than silicate systems, because its bonding is more covalent and does not require a calculation of long-range Coulombic interactions. 
Thanks to the rapid development of computer hardware and open source codes, running AIMD with hundreds of atoms can nowadays be achieved within a reasonable cost budget. The AIMD codes are free for academic research, the Linux operating system is free, and the appropriate parallel software package, Beowulf, is free also. We have assembled a small Beowulf cluster with thiry-one 2.1-GHz AMD Athlon processors Although the first AIMD simulation of a-SiC was carried out nearly a decade ago, current advances in the technique allow us to revisit this approach with far greater capability now. In addition, in order to perform various detailed structural and topological analyses and to study the structure variation under various forming conditions, e.g. different cooling procedures and/or different initial configurations, etc., we find we need the sort of accurate raw data provided by AIMD. Our further AIMD study of a-SiC may in turn help classic empirical-potential MD to refine its SiC potentials and validate its simulation results.

Earlier AIMD simulations [114] show that there may exist segregated graphitic regions in a-SiC assemblies [113]. The result is somewhat surprising considering that the simulation systems were very small, containing only 54-64 atoms, and were instantly quenched to $300 \mathrm{~K}$ from $4000 \mathrm{~K}$ with differing initial configuations. Nonetheless, electron paramagnetic resonance experiments [135] from about the same time show evidence for $s p^{2}$ carbon in a-SiC that supports the existence of graphitic regions. We believe this is a very interesting result and worthy of further investigation. A much larger-scaled AIMD simulation of a-SiC would definitely help to understand the formation and stability of a-SiC and validate this speculation. It is possible that a uniform a-SiC system may not be stable after all and might consist of many nanometersized or even sub-nanometer topologically segregated regions. However, such a study is impossible with classic MD, because its potential models cannot switch readily between different bonding configurations, for example between $s p^{2}$ and $s p^{3}$ carbons.

Another major discrepancy between classic MD and AIMD results lies in the assessment of chemical ordering. While one AIMD result [114] claimed that no chemical ordering exists in $\mathrm{SiC}$, i.e. the ratio $\chi$ of homonuclear to heteronuclear bond ratio $\chi$ is near 1.0, our classic MD simulations using Tersoff potentials suggest a strong chemical ordering, with $\chi$ between 0.3 and 0.6 [117,124]. Although it has been suggested [113] that the explanation for the discrepancy resides in the above-mentioned graphitic segregation, a detailed analysis of a large-scale AIMD-derived a-SiC should serve to test this suggestion and perhaps confirm the classic MD results.

\subsection{Simulation of Silicon Nitride Amorphization}

Silicon nitride $\left(\mathrm{Si}_{3} \mathrm{~N}_{4}\right)$ is a ceramic material of great interest due to its high melting temperature, high resistance to thermal shock, and superior mechanical properties, which explain its application in engine components and cutting tools. It has also been used in microelectronic devices as a gate dielectric and as a charge storage medium, owing to its high dielectric constant and large electronic band gap. Silicon nitride thin films are prepared by several processes: sputtering, chemical vapor deposition, and glow-discharge decomposition, which may leave the deposit in a nano-

crystalline or amorphous state. As electronic device become thinner, high quality films 
are required; understanding the microstructure and amorphizability of silicon nitride is therefore essential to the control of film deposition.

Silicon nitride has also been shown to be highly resistant to irradiation-induced amorphization. Amorphization in ion-implanted crystals occurs at deposited energy densities $~ 60 \mathrm{eV} /$ atom (0.9 dpa) [64], Table 1, but careful experiments with high energy ions, for which the displacement peak and ion stopping zone can be distinguishably separated, suggest that silicon nitride may not amorphize at all from displacements alone, in the absence of a chemical effect of the implanted ion [134].

\subsubsection{Topology of $\mathrm{Si}_{3} \mathrm{~N}_{4}$}

Crystalline silicon nitride comes in two polymorphic forms, $\alpha$ and $\beta$, which are topologically distinct. Both comprise vertex-sharing $\left[\mathrm{SiN}_{4}\right]$ tetrahedra connected three to a vertex; hence, $\mathrm{Si}_{3} \mathrm{~N}_{4}$ is intermediate between $\mathrm{SiO}_{2}$ and $\mathrm{SiC}$ in connectivity as a vertexsharing network. Its calculated structural freedom $f=-1.5$ indeed lies between that of $\mathrm{SiO}_{2}(f=0)$ and $\mathrm{SiC}$ ( $f=-3$ without admitting the possibility of antisite disorder). What distinguishes $\mathrm{Si}_{3} \mathrm{~N}_{4}$ from $\mathrm{SiC}$ is that, while Si-Si and N-N covalent bonds are stable, the $\mathrm{Si}_{\mathrm{N}}$ and $\mathrm{N}_{\mathrm{Si}}$ antisite configurations are not particularly stable. $\mathrm{Si}_{3} \mathrm{~N}_{4}$ therefore resembles $\mathrm{SiO}_{2}$ in its amorphization behavior but with considerably more constraints applicable. $\mathrm{Si}_{3} \mathrm{~N}_{4}$ can partially decompose with formation of $\mathrm{N}_{2}$ (the reverse of nitridation of silicon in $\mathrm{N}_{2}$ environments to synthesize $\mathrm{Si}_{3} \mathrm{~N}_{4}$ ), as apparently can $\mathrm{SiO}_{2}$-based networks [136], but such reactions are in principle reversible and subject to back reactions, hence to kinetic controls that may be unavailable to stochastic topological amorphization routes.

With $\left[\mathrm{SiN}_{4}\right]$ tetrahedra kept intact, the simpler of the two silicon nitride polymorphs, $\beta-\mathrm{Si}_{3} \mathrm{~N}_{4}$, has a single set of tetrahedron assembly rules and a simple local cluster of 27 tetrahedra comprising 3 3-rings, 8 4-rings and 20 6-rings [44]. The $\alpha$-form is topologically much more complex, with two inequivalent tetrahedron sites, correspondingly more complex assembly rules, and two local clusters of 29 and 40 tetarhedra respectively, comprising different numbers of 3-, 4-, 5-, 6- and 7 rings and dominated by 5-rings. In our modeling studies (proposed below) and experimental studies (outlined above) we will begin with the simpler $\beta-\mathrm{Si}_{3} \mathrm{~N}_{4}$ structure, with an awareness that an alternative crystalline arrangement with broader ring size distribution is also stable when evaluating the topology of amorphized assemblies.

Our previous attempts to model amorphized $\beta-\mathrm{Si}_{3} \mathrm{~N}_{4}$ using assembly-rule modifications [44] or tetrahedral bond-breaking and reassembly [105] yielded credibly amorphized structures $\left(\mathrm{Si}_{3} \mathrm{~N}_{4}\right.$-like local clusters) but with substantially more underconnection (37\%) than unoptimized a-silica models (27\%) and unrealistically high densities (3.42-3.65 instead of the $3.19 \times 10^{3} \mathrm{~kg} / \mathrm{m}^{3}$ for crystalline $\beta-\mathrm{Si}_{3} \mathrm{~N}_{4}$ ). Such assemblies are ripe for MD-optimization, as described in $\S 3$ for silica, coupled perhaps with global match reconstruction to minimize pre-optimization underconnection.

\subsubsection{Potentials for Silicon Nitride}

There are few empirical potential models for silicon nitride. Umesaki et al. [137] developed a model based on a two-body interaction consisting of a Coulombic and a repulsive term. Vashishta and co-workers [138-140] developed an empirical model that 
includes two- and three-body interactions. The latter potential has been tested by comparing structural properties from MD simulations with experimental data, and has been used to study fracture of $\mathrm{Si}_{3} \mathrm{~N}_{4}$ and $\mathrm{Si} / \mathrm{Si}_{3} \mathrm{~N}_{4}$ interfaces. However, both potential models undergo poor transferability. For example, the interface simulation used eight components to represent the $\mathrm{Si}$ and $\mathrm{N}$ species that experience different environments across the interface, along with a Stillinger-Weber potential to model the Si bulk. Consequently, MD simulations of silicon nitride with varying $\mathrm{N}$ contents are close to impractical. Mota et al. [141] developed a model based on Tersoff potential and claimed to be able to extend to a wide range of excess $\mathrm{N}$ contents. Collaborating with Dr. Shigenobu Ogata, a visiting scientist from Osaka University in Professor Sidney Yip's MIT group, we are using the Vashishta et al. and Mota et al. potential models in our MD refinement of silicon nitride and are seeking to validate them from the results of our topological analysis.

\subsubsection{Topological Analysis of MD-Refined Silicon Nitride}

MD simulations of $\mathrm{Si}_{3} \mathrm{~N}_{4}$ with up to several million atoms have been performed by Vashishta and his group [138-140] using massively parallel computational platforms. While mainly focused on the fracture and $\mathrm{Si} / \mathrm{Si}_{3} \mathrm{~N}_{4}$ interface simulations, they also demonstrated formation of amorphous $\mathrm{Si}_{3} \mathrm{~N}_{4}$. They have offered us coordinates for their a-Si $\mathrm{N}_{4}$, from which we are attemp;ting to deduce the local topologies using our ring search algorithms. We are also calculating the position of the first sharp electron diffraction peak for their assembly for comparison to experimental measurements of ionamorphized $\mathrm{Si}_{3} \mathrm{~N}_{4}$ (for which the chemical influence of the implanted ion appears necessary).

We are in addition conducting our own MD simulations of silicon nitride, using as starting positions topological models derived from local rules modifications (as in [44]); from tetrahedron separation, reorientation and rules-based reassembly (as in [105]); and from global match reconstructions (as done for $\mathrm{SiO}_{2}$ [84]). Because the intial topological modeling reconstructs so much of the assembly beforehand, the MD can be done quite economically, with modest computational platforms and without resort to massively parallel approaches.

We will additionally assess the effect of varying nitrogen content, evaluating compositions $\operatorname{SiN}_{\mathrm{x}}(\mathrm{x}=0-1.6)$ to explore the consequences of $\mathrm{N}_{2}$ loss or Si precipitation during irradiation or deposition. The influence of $\mathrm{N}$ content, topological disorder, and (possibly) chemical disorder, on density will likewise be explored. These MD simulations will be performed using various initial configurations, including random and crystalline, different equilibration temperatures, and gradual heating for thermophysical analysis as done for SiC.

Topological analysis of atomic coordination number, network ring size distribution, local cluster configurations, chemical disorder, homonuclear bond distribution, total correlation function, and first diffraction peak for all resulting arrangements is being carried out on all these simulations. Lastly, the effect of $\mathrm{N}$ content on amorphizability of silicon nitride in being investigated from both topological and chemical disorder points of view. 


\subsubsection{Modeling Aluminum Nitride Amorphization}

As an intriguing corollary to the proposed silicon nitride study and our prior exploration of $\mathrm{SiC}$, amorphization of a nitride with the same stoichiometry and crystal structure as silicon carbide, but without its antisite disorder facility, is being investigated. Aluminum nitride (AlN) has the hexagonal wurtzite structure of $\alpha$-SiC, with each $\mathrm{Al}$ or $\mathrm{N}$ atom tetrahedrally coordinated by four atoms of the opposite species. Growth of AlN crystal has been difficult because of the decomposition of the material at temperatures approaching the melting point, and there are few simulations of AlN structure, except for some $a b$ initio lattice dynamics simulations [142]. Currently, there is no suitable empirical potentials for the AlN structure, which makes MD modeling of AlN a difficult task. It is, however, possible to use $a b$ initio molecular dynamics to simulate the AlN structure.

\subsection{Structural Simulations of Amorphous Silica}

Despite our extensive topological and MD modeling of silica, described in §3, and the experimental diffraction work proposed in §5.1.1, there still remain several silica modeling projects we are intent on completing. These concern paracrystallinity, ring enumerations, and a classic density anomaly.

\subsubsection{Paracrystallinity in Amorphous Silicas}

The question of whether amorphous silica $\left(\mathrm{a}-\mathrm{SiO}_{2}\right)$ is a continuous random network (CRN [143]) or a paracrystalline assemblage (PC [144-146]) has been debated for decades and is still not settled. However, with the help of topological analysis, particularly the local primitive ring cluster (LRC) concept proposed by Marians and Hobbs [83], we are attempting to resolve the issue by carefully designed simulations.

One of the difficulties in distinguishinging between CRN and PC arrangements is the scale of paracrystalline units. While the Si-tetrahedron $\left[\mathrm{SiO}_{4}\right]$ is the basic unit for a$\mathrm{SiO}_{2}$ and all compact crystalline silica polymorphs but stishovite, the Si-centered LRC is the smallest topological unit to differentiate crystalline from amorphous silica structures. We have repeatedly $[45,83,85]$ pointed out that LRC is the topological equivalent of a unit cell. As examples, the Si-centered LRC in cristobalite comprises twelve 6-rings and involves 87 atoms, in quartz six 6-rings and 40 8-rings and involves a total of 189 atoms, and in coesite a wide range of ring sizes from 4-rings to 12-rings over two non-equivalent Si sites involving 225 and 243 atoms.

Our investigation implements an earlier suggestion by Marians [147] that vitreous silica could be thought of as cristobalite-like 6-ring local cluster regions embedded in a glue of quartz-like 8-ring connective material. In the simulations, we engineer a series of structures with various numbers, sizes and kinds of these LRCs distributed throughout an amorphous matrix (for example, the fairly random 5000-K configuration in Fig. 11c); relax the assemblies under various conditions; and compute their total correlation functions, first sharp diffraction peaks, and fluctuation microscopy signatures. These latter three correlative functions, thus obtained for various LRC distributions, are 
compared to experimental values in order to validate either CRN or PC predictions. We then compare the same diagnostics obtained for the cristobalite-like, quartz-like and 5000-K random MD-equilibrated amorphous silica structures discussed in §3.2.2 and illustrated in Fig. 11.

\subsubsection{Ring-Circuit Enumeration in Local Ring Clusters}

Different local ring enumeration schemes (e.g. [148-151]) have been proposed beside that introduced by Marians and Hobbs [83], and we consider it important to establish their equivalence. For example, a disparity we found [85] with the Goetzke and Klein [149] primitive ring accounting is actually attributable (Goetzke, private communication, 2001) to their choice to exclude translationally equivalent or symmetryequivalent rings; we prefer to include all primitive rings, whether crystallographically equivalent or not in crystalline polymorphic forms, because of our primary focus on aperiodic structures.

Recently, Treacy et al. [150-151] proposed an altogether different approach to local ring enumeration in network structures that groups rings according to pairs of atom neighbors for each atom. For example, the Si atom in quartz is four-coordinated and has six pairs of neighbors, which leads to a ring complement given by the expression (employing circuit symbols) 6: 6: $6_{2}: 6_{2}: 8_{7}: 8_{7}$, where $6_{2}$ means there are two 6-rings that go through the center $\mathrm{Si}$ and a certain pair of its neighbors. The rings (or circuits) examined in this approach, termed fundamental circuits by Treacy et al., are just the primitive rings defined by Marians and Hobbs [83]: circuits for which there is no shorter path between any two atoms on the ring (circuit) than the path that lies on the ring (circuit).

The approach appears to encounter problems in application to more complex amorphous structures, however, despite its success with simple crystal structures. This is because the way Treacy et al. group circuits does not comply with the circuit definition. As argued by Guttman [148], there may be no fundamental circuit involving a certain pair of neighbors of a central atom, or there may be more than one fundamental circuit for a certain pair of neighbors of a central atom. While it is possible to extend the Treacy et al. expressions, such as NC:NC:6 $6_{2}: 6_{2}:\left(6_{3}, 20,40\right): 8_{7}$ where two pairs have no fundamental circuits (NC) and one pair has five such circuits with various sizes, we do not see much advantage to using this description in preference to the simpler ring distribution with respect to ring size as $67: 8_{7}: 20: 40$. Therefore, we plan to continue to employ our own LRC enumeration scheme.

We have used NC for no fundamental circuit instead of symbol $\infty$ as suggested by Treacy et al. because use of symbol $\infty$ could be misleading, as it suggests an "open" circuit with no ring for that pair. In fact, we note that there are two possibilities for no fundamental circuit: 1) pairs that do have Wells-type [152] circuits but just do not have O'Keeffe-type [153] fundamental circuits; 2) pairs that do not have any type of circuit up to a quite large size $\mathrm{K}$ (open circuit).

Treacy et al. also claim to have discovered a much simpler algorithm for searching for fundamental circuits. Their algorithm is also problematic. We take as an example the 12-ring in Fig. 29 (their Fig. 1c in ref. [151]). Clearly, the 12-ring is a fundamental circuit (primitive ring). We list below the breadth-first search using $\mathrm{p}$ and $\mathrm{q}$ 
values (the numbers of the coordination shell around a chosen central vertex) centered on vertices 1 and 2 for the 12-ring:

\begin{tabular}{|c|c|c|c|c|c|c|c|c|c|c|c|c|}
\hline Vertex of 12-ring & 1 & 2 & 23 & 22 & 21 & 20 & 19 & 18 & 17 & 16 & 15 & 3 \\
\hline p-value on atom 1 & 0 & 1 & 2 & 3 & 4 & 5 & 6 & 5 & 4 & 3 & 2 & 1 \\
\hline q-value on atom 2 & - & 0 & 1 & 2 & 3 & 4 & 5 & 6 & 7 & 6 & 5 & 4 \\
\hline
\end{tabular}

Here vertex 19 is the aponode, the furthest vertex on the circuit from the central vertex. The q-value of vertex 3 is shortcut by path 2-10-4-6-3. As described in the "additional test of step 6" in Treacy et al. [151], for early vertices before the aponode, $\mathrm{p}=\mathrm{q}+1$, which is true. However, for the later vertices after aponode 19, the test fails for $p+q=$ $\mathrm{p}_{\mathrm{a}}+\mathrm{q}_{\mathrm{a}}$, where $\mathrm{p}_{\mathrm{a}}+\mathrm{q}_{\mathrm{a}}$ are the values at the aponode. Therefore, the test incorrectly regards the 12-ring as a non-fundamental circuit.

As a consequence, the Si-centered LRC in quartz calculated using this algorithm is incorrect. It has been definitively established in several studies $[83,85,112]$ that the LRC in quartz comprises six 6-rings and 40 8-rings. However, the Treacy et al. study claims that $\mathrm{Si}$ in quartz has the ring sequence $6: 6: 6_{2}: 6_{2}: 8_{7}: 8_{7}$ with weight of 63 , which means they find six 6-rings and only 14 8-rings; 26 8-rings are missed by this algorithm. Furthermore, their concept of "circuit flux" (the number of circuits that pass through each vertex) proves inadequate; it fails at least when there is a shortcut from the center vertex to one of the vertices on the circuits, e.g., the 4-1-5 pair in Fig. 29.

We nevertheless continue to investigate alternative ring enumeration schemes in well-connected networks to test against the robustness of those algorithms we have already developed.

\subsubsection{Simulation of the Silica Anomaly using a Topological Approach}

It is well known that fast-cooled silica has a higher density than slowly cooled silica, an observation known as the silica anomaly. This phenomenon has been successfully reproduced in MD simulation of silica structures [91]. However, less has been done to examine the phenomenon in greater detail. In our MD simulation study, we cool down a silica melt to room temperature with various cooling rates, choose several instantaneous structures at various temperatures, particularly around the transition range and anomaly points, instantly quench them to zero temperature, and then study their structures with analysis of CN, LRC, and other topological parameters. We find that changes in local structure (atom coordination) as well as intermediate-range structure (ring configurations) play an important role. Our current experiments are helping to understand this phenomenon on a microscopic scale.

Our presumption is that the silica anomaly is caused by the transition of Si from a higher coordination (5-fold, 6-fold) at high temperature to four-coordination at low temperature. If this assumption holds true, we should expect the CN transition to parallel the density anomaly. By this reasoning, Si atoms in alkali silicates should always be 4coordinated, even in the melt, because these compounds do not exhibit the silica anomaly. In the course of carrying out the MD simulations, we are exploring modifications to the BKS potential we use in an attempt to improve the Si-Si interaction term. 


\subsection{Topological-MD Modeling of Irradiation Amorphized Silicon}

Silicon is a major component in semiconductor devices for electronic communications and control systems and in microelectromechanical device systems (MEMS) used in chemical sensing and electrical and mechanical control. These devices find widespread use in applications (space, military and defence, nuclear reactors, nuclear waste monitoring) with the potential for significant radiation exposure [1]. "Radiation hardening" is an approach traditionally used in the design of electronics for satellite systems, and typically involves protection against spurious generation of charge carriers in ionizing irradiation fields; but in MEMS applications, small changes in mechanical properties such as dimension and elasticity can degrade device performance, and it is less clear how to protect such devices from mechanical degradation in irradiation fields. The primary irradiation events in the latter case are single displacements (proton and electron irradiation fields) and more extension collision cascade events in the case of neutron flux or high energy ions.

The nature and structure of the resulting damage (point defect, defect clusters and extended defects, amorphous zones) is important to understand in predicting the mechanical response, particularly for small irradiation fluences below those at which responses can be measured experimentally by even sensitive conventional techniques such as triple-crystal X-ray diffraction [M. Treska and L. W. Hobbs, to be published] and acoustic spectoscopy [154; H.-Z. Wu, L. W. Hobbs and G. A. D. Briggs, to be published]. Silicon has long been known to amorphize under selected ion implantation conditions, depending on temperature and ion flux, mass and energy. The collision cascades responsible have been variously modeled in MD simulations of up to 1 million atoms [155-158], from which it has been concluded that $5 \mathrm{keV}$ ions at least as light as $\mathrm{Ar}$ could leave behind amorphous regions large enough to be stable up to 400-500 K. Neutron irradiation of Si (Si self-ion PKAs) has been observed to produce amorphous regions at $300 \mathrm{~K}$ [159]. The most recent MD model [158], using the environmentallydependent potential (EDIP) [50], has demonstrated that silicon likely amorphizes through growth of such locally amorphous zones.

\subsubsection{Topological-MD Simulation of Amorphous Zones in Silicon}

Apart from the studies of Treacy, Voyles, Gibson et al. discussed in §5.4.2, there has been little topological modeling of the amorphous state of silicon, and none applied to radiation-induced amorphization. Treacy et al. [150] applied our concept of the local cluster to coordinates from six computer models (four continuous random network models, two paracrystalline assemblies) of a-Si and have presented ring statistics and a limited local cluster analysis for each. With respect to radiation-induced amorphization, the density of metamict silicon has been estimated at $-1.7 \%$ below that of crystalline $\mathrm{Si}$ [160], while a percolation rigidity transition has been predicted at a bond-rupture density corresponding to an average Si coordination $\langle\mathrm{CN}\rangle=2.4$ [161]. We are investigating more fully the topology of amorphized silicon analogously to the manner described in 
$\S \S 3$ and 4 for $\mathrm{SiO}_{2}$ and $\mathrm{SiC}$. Using several different topological generation algorithms, we apply the EDIP to optimize the topologically-generated assemblies using MD.

As noted in $\$ 2.2 .2$, the diamond structure of crystalline silicon shares the same topology as cristobalite $\mathrm{SiO}_{2}$ and the same structural freedom $f \sim 0$. This fact provides a potentially intriguing way to simulate a-Si: we amorphize an ideal $\beta$-cristobalite model, as outlined in $\S 3$ using $\beta$-cristobalite re-assembly rules to favor $180^{\circ} \mathrm{Si}-\mathrm{O}$-Si intertetrahedral angles, then remove the oxygen atoms which sit at the $\mathrm{Si}-\mathrm{Si}$ bond midpoints. The result is an amorphous $\mathrm{Si}$ model, like our cristobalite-like a- $\mathrm{SiO}_{2}$ dominated by 6rings, whose atom positions and topologies can be refined by subsequent MD. The global-match algorithm can likewise be applied to a disordered Si atom assembly. Both models are equilibrated using MD methods, as we have done for amorphized $\mathrm{SiO}_{2}$, and compared in their topologies with each other and with other a-Si models and previous MD-cascade results.

Previous MD cascade simulations have been based mainly on Stillinger-Weber [162] and Tersoff [163] potentials. The Stillinger-Weber potential heavily penalizes coordinations other than tetrahedral, while the Tersoff potential generates an overly high melting point of $2400 \mathrm{~K}$. The former could contribute to over-recrystallization of disordered $\mathrm{Si}$, the latter to resistance to local melting. The environmentally-dependent interatomic potential for Si [50] provides a more realistic melting point (1550 K; the experiemntal value is $1685 \mathrm{~K}$ ), and its functional form contains coordination dependence, making it a good candidate for use in collision cascade reconstructions in which local melting and sudden quenching into disordered states play important roles.

\section{REFERENCES}

1. F. W. Clinard and L. W. Hobbs, "Radiation effects in non-metals," Chapter 7 in Physics of Radiation Effects in Crystals, ed. R. A. Johnson and A. N. Orlov (Elsevier Science Publishers, Amsterdam, 1986) pp. 387-471.

2. L. W. Hobbs, F. W. Clinard, Jr., S. J. Zinkle and R. C. Ewing, "Radiation effects in ceramics,” J. Nucl. Mater. 216 (1994) 291-321.

3. D. Steiner, organizer., DOE Workshop on Ceramic Composities under Irradiation, Rensselaer Polytechnic Institute, Troy, NY, 11-13 July 1994.

4. W. J. Weber, R. C. Ewing, C. A. Angell, G. W. Arnold, A. N. Cormack, J-M. Delaye, D. L. Griscom, L. W. Hobbs, A. Navrotsky, D. L. Price, A. M. Stoneham and M. C. Weinberg, "Radiation effects in glasses used for immobilization of highlevel waste and plutonium disposition,” DOE Council on Materials Sciences Panel Workshop on Radiation Effects in Glasses for the Immobilization and Disposal of High-Level Radioactive Wastes and Excess Weapons Plutonium," Santa Fe, NM, 2529 February 1996; J. Mater. Res. 12 (1997) 1946-78. 
5. W. J. Weber, R. C. Ewing, C. R. A. Catlow, T. Diaz de la Rubia, L. W. Hobbs, C. Kinoshita, Hj. Matzke, A. T. Motta, M. A. Nastasi, E. H. K. Salje, E. R. Vance and S. J. Zinkle, "Radiation effects in crystalline ceramic phases relevant to the immobilization and disposition of nuclear waste and weapons plutonium," DOE Council on Materials Sciences Panel Workshop on Radiation Effects in Crystalline Ceramic Phases Relevant to the Immobilization and Disposition of Nuclear Waste and Weapons Plutonium, Lihue, HI, 12-17 January 1997; J. Mater. Res.. 13 (1998) 1434-84.

6. R. C. Ewing, organizer, Workshop on Amorphization of Ceramics, Santa Fe, NM, July 2000.

7. R. C. Ewing, Nucl. Instrum. Meth. B 91 (1994) 22.

8. L. W. Hobbs, "Network topology of aperiodic networks," J. Non-Cryst. Solids 192\&193 (1995) 79-91.

9. A. T. Motta, J. Nucl. Mater. 244 (1997) 227-50.

10. L. W. Hobbs, A. N. Sreeram, C. E. Jesurum and B. A. Berger, "Structural freedom, topological disorder, and the irradiation-induced amorphization of ceramic structures," Nucl. Instrum. Meth. B 116 (1996) 18-25.

11. Linn W. Hobbs, C. Esther Jesurum and Bonnie Berger, "Rigidity constraints in amorphization of singly- and multiply-polytypic structures," in Rigidity Theory and Applications, ed. M. F. Thorpe and P. M. Duxbury (Kluwer/Plenum, New York, 1999) pp.191-215.

12. Linn W. Hobbs, C. Esther Jesurum and Bonnie Berger, "Rigidity constraints in amorphization of multiply-polytopic multiply-connected ceramic structures," Mat. Res. Soc. Symp.Proc. 540 (2000) 717-28.

13. P.K. Gupta and A. R. Cooper, J. Non-Cryst. Solids 123 (1990) 14; P. K. Gupta, J. Amer. Ceram. Soc. 76 (1993) 1088.

14. D. Jacobs and M. F. Thorpe, Phys. Rev. E53 (1996) 3682; M. F. Thorpe, B. D. Djordjevic and D. J. Jacobs, "The structure and mechanical properties of networks," in: Amorphous Insulators and Semiconductors, ed. M. F. Thorpe and M. I. Mitkova (Kluwer Academic Publishers, Dordrecht, Netherlands, 1997) p. 289.

15. W. Primak, Phys. Rev. 110 (1958) 1240; W. Primak and R. Kampwirth, J. Appl. Phys. 39 (1968) 5651.

16. W. J. Weber, R. C. Ewing and Lu-Min Wang, J. Mater. Res. 9 (1994) 688. 
17. W. J. Weber and Hj. Matzke, Radiat. Eff. 98 (1986) 93; R. C. Ewing, R. F. Haaker and W. Lutze, in: Scientific Basis for Nuclear Waste Management V, ed. W. Lutze, Mat. Res. Soc. Symp. Proc. 11 (1982) 389.

18. W. J. Weber, J. Mater. Res. 5 (1990) 2687; Radiat. Eff. 115 (1991) 341; J. Amer. Ceram. Soc. 76 (1993) 1729.

19. H. Inui, H. Mori, T. Sakata and H. Fujita, J. Non-Cryst. Solids 116 (1990) 1.

20. C. K. Kinoshita, Nihon Genshiryoku Gakkaishi 28 (1986) 1009.

21. D. E. Luzzi and M. Meshii, Res. Mechanica 21 (1987) 207.

22. L. W. Hobbs and M. R. Pascucci, "Radiolysis and defect structure in electron irradiated $\alpha$-Quartz,"J. Physique 41 [C6] (1980) 237.

23. L. W. Hobbs, "Electron beam sensitivity in inorganic specimens," Ultramicroscopy 23 (1987) 339; "Murphy's Law and the uncertainty of electron probes,"Scanning Microscopy, Suppl. 4 (1990) 171-83.

24. J. Rankin, L. A. Boatner, C. W. White and L. W. Hobbs, “A cross-sectional TEM study of the effects of annealing conditions on the regrowth of lead-implanted single crystal calcium titanate,”Mat. Res. Soc. Symp. Proc. 100 (1988) 453-58.

25. J. Rankin, L. W. Hobbs, L.A. Boatner and C. W. White, "An in-situ annealing study of lead-implanted single crystal calcium titanate,” Nucl. Instrum. Meth. Phys. Res. B32 (1988) 28-31.

26. F. W. Clinard, Jr., D. E. Peterson, D. L. Rohr and L. W. Hobbs, "Self Irradiation Effects in ${ }^{238} \mathrm{Pu}$-Substituted Zirconolite: I. Temperature Dependence of Damage," $J$. Nucl. Mater. 126 (1984) 245-54.

27. L. M. Wang, S. X. Wang, W. L. Gong, R. C. Ewing and W. J. Weber, "Amorphization of ceramic materials by ion beam bombardment," Mat. Sci. Eng. A253 (1998) 106-113.

28. G. Van Tendeloo, J. Van Landuyt and S. Amelinckx, phys. stat. solidi A33 (1976) 723.

29. W. J. Weber and L. M. Wang, Nucl. Instrum. Meth. B106 (1995) 298; W. J. Weber, L. M. Wang and N. Yu, Nucl. Instrum. Meth. B116 (1996) 322.

30. P. K. Gupta, J. Non-Cryst. Solids 195 (1996) 158.

31. R. A. B. Devine, Nucl. Instrum. Meth. B91 (1994) 378-80. 
32. R. A. Weeks and D. L. Kinser, eds., Effects of Modes of Formation on the Structure of Glasses (Trans. Tech Publications, Aedermannsdorf, Switzerland, 1987); Diffusion \& Defect Data 53\&54 (1988).

33. C. E. Jesurum, V. Pulim and L. W. Hobbs, "Modeling the cascade amorphization of silicas with local-rules reconstruction," Nucl. Instrum. Meth. B114 (1998) 25-34.

34. L. C. Qin and L. W. Hobbs, "Energy-filtered electron diffraction study of vitreous and amorphized silicas,"J. Non-Cryst. Solids 192\&193 (1995) 456-62.

35. S. X. Wang, L. M. Wang, R. C. Ewing and R. H. Doremus, J. Non-Cryst. Solids 238 (1998) 198-213.

36. S. X. Wang, L. M. Wang, R. C. Ewing and R. H. Doremus, J. Non-Cryst. Solids 238 (1998) 214.

37. M. J. Caturla, T. Díaz de la Rubia, L. A. Marqués and G. H. Gilmer, "Ion-beam processing of silicon at keV energies: a molecular dynamics study,” Phys. Rev. B54 (1996) 16683-95.

38. J.-M. Delaye and D. Ghaleb, Nucl. Instrum. Meth. B153 (1999) 157; Phys. Rev. B61 (2000) 14481.

39. R. Devanathan, W. J. Weber and T. Díaz de la Rubia, "Computer simulation of a 10 keV Si displacement cascade in SiC,” Nucl. Instrum. Meth. B141 (1998) 118-22.

40. L. Malerba, J. M. Perlado, A. Sánchez-Rubio, I. Pastor, L. Colombo and T. Díaz de la Rubia, "Molecular dynamics simulation of defect production in irradiated $\beta$-SiC," J. Nucl. Mater. 276 (2000) 235-42.

41. F. Gao and W. J. Weber, "Atomic-scale simulation of $50 \mathrm{keV}$ Si displacement cascades in $\beta$-SiC,” Phys. Rev. B63 (2000) 54101-1-7.

42. W. J. Weber, "Models and mechanisms of irradiation-induced amorphization in ceramics,” Nucl. Instrum. Meth. Phys. Res. B166-167 (2000) 98-106.

43. Rodney C. Ewing, “The metamict state: 1993--the centennial,” Nucl. Instrum. Meth. Phys. Res. B91 (1994) 22-29.

44. C. E. Jesurum, V. Pulim and L. W. Hobbs, "Topological modeling of amorphized tetrahedral ceramic network structures,” J. Nucl. Mater. 253 (1998) 87-103.

45. Linn W. Hobbs, C. Esther Jesurum and Bonnie Berger, "The topology of silica networks," Chapter 1 in: Structure and Imperfections in Amorphous and Crystalline Silica, ed. J.-P. Duraud, R. A. B. Devine and E. Dooryhee (John Wiley \& Sons, London, 2000) pp. 3-47. 
46. L. W. Hobbs and X. Yuan, "Topology and topological disorder in silica," in: Defects in $\mathrm{SiO}_{2}$ and Related Dielectrics: Science and Technology, ed. G. Pacchioni, L. Skuja and D. Griscom (Kluwer, Dordrecht, The Netherlands, 2000) 37-71.

47. A. N. Cormack and Yuan Cao, "Molecular dynamics simulation of silicate glasses," in Modelling of Minerals and Silicated Materials, ed. B. Silvi and P. D'Arco (Kluwer, Netherlands, 1997) p. 227-71.

48. Xianglong Yuan and Alistair N. Cormack, "Local structures of MD-modeled vitreous silica and sodium silicate glasses,” J. Non-Cryst. Solids 283 (2001) 69-87.

49. Clark L. Allred, Jeffrey T. Borenstein and Linn W. Hobbs, "Nuclear radiationinduced dimensional changes in MEMS Si and glass substrates," Mater. Res. Soc. Symp. B: Materials science of Microelectromechanical Systems (MEMS) Devices IV (Boston, MA, 26-30 November 2001).

50. M. Z. Bazant, E. Kaxiras and J. F. Justo,, "Environment-dependent interatomic potential for bulk silicon,” Phys. Rev. B56 (1997) 8542.

51. Meijie Tang and Sidney Yip, "Atomic size effects in pressure-induced amorphization of a binary covalent lattice,” Phys. Rev. Lett. 75 (1995) 2738-41.

52. Meijie Tang and Sidney Yip, "Atomistic simulation of thermomechanical properties of $\beta$-SiC,” Phys. Rev. B52 (1995) 15150-59.

53. C. S. Jayanthi, M. Tang, S. Y. Wu, J. A. Cocks and S. Yip, "Local analysis of structural instability in stressed lattices: crack nucleation in a covalent solid," Phys. Rev. Lett. 79 (1997) 4601-04.

54. Lisa J. Porter, Ju Li and Sidney Yip, "Atomistic modeling of finite temperature properties of $\beta$-SiC. I. Lattice vibrations, heat capacity, and thermal expansion," $J$. Nucl. Mater. 246 (1997) 53-59.

55. $\mathrm{Ju} \mathrm{Li}$, Lisa Porter and Sidney Yip, "Atomistic modeling of finite temperature properties of $\beta$-SiC. II. Thermal conductivity and effects of point defects," J. Nucl. Mater. 225 (1998) 139-52.

56. P. K. Gupta, J. Amer. Ceram. Soc. 76 (1993) 1088.

57. C. J. McHargue, P. S. Sklad and C. W. White, Nucl. Instrum. Meth. Phys. Res. B46 (1990) 79.

58. Hj. Matzke and L. M. Wang, J. Nucl. Mater. 231 (1996) 155.

59. K. E. Sickafus, N. Yu and M. Nastasi, Los Alamos Nat. Lab. Report LA-UR 96-1382 (1996). 
60. R. Devanathan, W. J. Weber, K. E. Sickafus, et al., Nucl. Instrum. Meth. Phys. Res. B141 (1998) 398.

61. A. Meldrum, L. A. Boatner and R. C. Ewing, Nucl. Instrum. Meth. Phys. Res. B141 (1998) 353.

62. T. Hartmann, W. J. Weber, N. Yu, K. E. Sickafus, J. N. Mitchell, C. J. Wetteland, M. G. Hollander and M. Nastasi, Nucl. Instrum. Meth. Phys. Res. B141 (1998) 398.

63. W. J. Weber, N. Yu and L. M. Wang, "Irradiation-induced amorphization in $\beta$-SiC," J. Nucl. Mater. 253 (1998) 53-59.

64. W. Bolse, S. D. Peteves and F. W. Saris, Appl. Phys. A58 (1994) 493.

65. A. Meldrum. L. A. Boatner and R. C. Ewing, Nucl. Instrum. Meth. Phys. Res. B141 (1998) 353.

66. H. Abe, H. Naramoto and C. Kinoshita, Mater. Res. Soc. Symp. Proc. 373 (1995) 383.

67. J. K. N. Lindner, R. Zuschlag and E. H. Te Kaat, Nucl. Instrum. Meth. Phys. Res. B62 (1992) 314.

68. R. K. Eby, R. C. Ewing and R. C. Birtcher, J. Mater. Res. 7 (1992) 3080.

69. A. N. Sreeram, L. W. Hobbs, N. Bordes and R. C. Ewing, Nucl. Instrum. Meth. Phys. Res. B116 (1996) 126.

70. A. N. Sreeram and L. W. Hobbs, Mater. Res. Soc. Symp. Proc. 279 (1993) 559; 321 (1994) 26.

71. N. Yu, K. E. Sickafus and M. Nastasi, Philos. Mag. Lett. 70 (1994) 235

72. K. E. Sickafus, N. Yu and M. Nastasi, Nucl. Instrum. Meth. Phys. Res. B116 (1996) 85.

73. L. L. Snead and S. J. Zinkle, Mater. Res. Soc. Symp. Proc. 439 (1997) 595-606; S. J. Zinkle and L. L. Snead, Mater. Res. Soc. Symp. Proc. 540 (1999) 302

74. F. Gao and W. J. Weber, "Computer simulation of disordering and amorphization by Si and Au recoils in 3C-SiC,” J. Appl. Phys. 89 (2001) 4275-81; F. Gao, W. J. Weber and R. Devanathan, "Atomic-scale simulation of displacement cascades and amoprhization in $\beta$-SiC,” Nucl. Instrum. Meth. B180 (2001) 176-86.

75. W. Bolse, J. Conrad, F. Harbsmeier, M. Borowski and T. Rodle, Mat. Sci. Forum 248/249 (1997) 319. 
76. W. Bolse, "Formation and development of disordered networks in Si-based ceramics under ion bombardment,” Nucl. Instrum.. Meth. B141 (1998) 131.

77. W. Bolse. Nucl. Instrum. Meth. Phys. Res. B148 (1999) 83.

78. H. Inui, H. Mori and T. Sakata, Philos. Mag. B65 (1992) 1; B66 (1992) 737.

79. M. T. Dove, V. Heine and K. D. Hammonds, Min. Mag. 59 (1995) 629; in [11], p. 349.

80. W. J. Weber, R. C. Ewing and A. Meldrum, J. Nucl. Mater. 250 (1997) 147.

81. A. C. Wright, "Neutron and X-ray amorphography,” in Experimental Techniques in Glass Science, ed. C. J. Simmons and O. H. El-Bayoumi (American Ceramic Society, Westerville, OH, 1993) pp. 205-314.

82. A. C. Wright, "The structure of vitreous silica: What have we learned from 60 years of diffraction studies?,” J. Non-Cryst. Solids 179 (1994) 84-115.

83. C. S. Marians and L. W. Hobbs, "Network properties of crystalline polymorphs of silica,” J. Non-Cryst. Solids 124 (1990) 242-53; C. S. Marians and L. W. Hobbs, “Local structure of silica glasses,” J. Non-Cryst. Solids 119 (1990) 269-82.

84. Linn W. Hobbs, C. Esther Jesurum, Alexander Coventry, Vinay Pulim, Russell Schwartz and Bonnie Berger, "Towards a topological description of poorlycrystalline networks," in: Advanced Materials for the21st Century: The Julia R. Weertman Symposium, ed. Y. W. Chung, D. Durand, P. Liaw and G. Olson (TMS Publications, Warrendale, PA, 1999) pp. 475-86.

85. L. W. Hobbs, C. E. Jesurum and B. Berger, "Local topology of silica networks," Philos. Mag. A78 (1998) 679-711.

86. C. E. Jesurum, V. Pulim and L. W. Hobbs, "Modeling the cascade amorphization of silicas with local-rules reconstruction,” Nucl. Instrum. Meth. B114 (1998) 25-34.

87. H. N. Gabow, “An efficient implementation of Edmond's algorithm for maximum matching on graphs,” J. Assoc. Comp. Mach. 23 (1975) 221-34.

88. Xianglong Yuan, Vinay Pulim and Linn W. Hobbs, “Molecular dynamics refinement of topologically generated reconstructions of simulated irradiation cascades in silica networks,” J. Nucl. Mater. 289 (2001) 71-79; erratum, J. Nucl. Mater. 295 (2001) 132.

89. X. Yuan, V. Pulim and L. W. Hobbs, "Refinement of topologically modeled cascade-amorphized silicas using molecular dynamics simulation," in Microstructural Processes in Irradiated Materials, ed. G. E. Lucas, L. Snead, M. A. 
Kirk, Jr. and R. G. Elliman (Materials Research Society, Warrendale, PA, 2001); Mat. Res. Soc. Symp. Proc. 650 (2001) R3.18 1-6.

90. B. W. H. van Beest, G. J. Kramer and R. A. van Santen, "Force fields for silicas and aluminophosphates based on ab initio calculations," Phys. Rev. Lett. 64 (1990) 1955.

91. K. Vollmayr, W. Kob and K. Binder, Phys. Rev. B54 (1996) 15808.

92. Xianglong Yuan and Alastair N. Cormack, "Molecular dynamics simulation of surface structures of silica and silicate glasses," J. Non-Cryst. Solids 27 (2001) 2837.

93. W. Smith and T. R. Forester, http://www.dl.ac.uk/tcsc/software/DL_POLY (last accessed 2001).

94. H. J. C. Berendsen, J. P. M. Postma, W. van Gunster, A. DiNala and J. R. Haak, J. Chem. Phys. 81 (1984) 3684.

95. Xianglong Yuan, "Molecular dynamics simulation of silicate glasses and their surfaces,” Ph.D. dissertation (Alfred University, Alfred, NY, 1999).

96. S. R. Elliott, "Extended-range order, interstitial voids, and the first sharp diffraction peak of network glasses,” J. Non-Cryst. Solids 182 (1995) 40-48; Adv. Phys. 38 (1989) 1.

97. Linn W. Hobbs and Bonnie Berger, "Radiation-induced topological disorder in irradiated ceramics and glasses," renewal proposal for DOE Grant DR-FG0289ER45396 (October 1997).

98. S. J. Zinkle and L. L. Snead, "Influence of irradiation spectra and implanted ions on the amorphization of ceramics,” Nucl. Instrum. Meth. B116 (1996) 92-101.

99. W. Wesch, Nucl. Instrum. Meth. B116 (1996) 305.

100. M. A. Capano and R. J. Trew, MRS Bull. 22 (1997) 19.

101. E. Wendler, A. Heft and W. Wesch, Nucl. Instrum. Meth. B141 (1998) 105.

102. W. J. Weber, L. M. Wang, N. Yu and N. J. Hess, Mater. Sci. Eng. A253 (1998) 62.

103. L.L. Snead and J. C. Hay, J. Nucl. Mater. 273 (1999) 213.

104. Fei Gao and William J. Weber, "Molecular dynamic simulation of cascade overlap and amorphization in 3C-SiC,” Mat. Res. Soc. Symp. Proc. 650 (2001) R3.20 1-6. 
105. L. W. Hobbs, C. E. Jesurum and V. Pulim, "Topological modeling of cascade amorphization in network structures using local rules,” Mater. Sci. Eng. A253 (1998) 16-29.

106. R. Devanathan, W. J. Weber and T. Diaz de la Rubia, "Atomistic simulation of defect production in $\beta$-SiC,” Mat. Res. Soc. Symp. Proc. 504 (1998) 45-50.

107. R. Devanathan and W. J. Weber, "Displacement energy surface in 3C and 6H SiC," J. Nucl. Mater. 278 (2000) 258-65.

108. F. Gao, W. J. Weber and W. Jiang, "Primary damage states produced by Si and Au recoils in SiC: a molecular dynamics and experimental investigation,” Phys. Rev. B63 (2001) 214106-1-6.

109. F. Gao, Ram Devanathan and W. J. Weber, "Si displacement cascades revealed by atomic-scale simulations in 3C-SiC,” Fusion Tech. 39 (2001) 574-78.

110. R. Devanathan, F. Gao and W. J. Weber, "Computer simulation of energy dependence of primary damage states in SiC," Mat. Res. Soc. Symp. Proc. 650 (2001) 3.22 1-6.

111. Fei Gao and William J. Weber, "Empirical potential approach for defect properties in 3C-SiC," Nucl. Instrum. Meth. B (in press, 2001); F. Gao, C. J. Bylaska, W. J. Weber and R. Corrales, "Native defect properties in $\beta$-SiC: Ab-initio and empirical potential calculations," Nucl. Instrum. Meth. B180 (2001) 286-92.

112. X. Yuan and A. N. Cormack, "Highly efficient algorithm for primitive ring statistics in topological networks,” Comp. Mater. Sci. 24 (2002) 12-18.

113. J. Tersoff, Phys. Rev. B49 (1994) 16349.

114. F. Finocchi, G. Galli, M. Parrinello and C. M. Bertoni, Phys. Rev. Lett. 68 (1992) 3044.

115. P. C. Gehlen and J. B. Cohen, Phys. Rev. A139 (1965) 844.

116. C. P. Flynn, Point Defects and Diffusion (Oxford Univ. Press, 1972) pp. 55-59.

117. Xianglong Yuan and Linn W. Hobbs, "Influence of interactomic potentials in MD invetigation of ordering in a-SiC," in" Microstructural Processes in Irradiated Materials, ed. G. E. Lucas, L. Snead, M. A. Kirk, Jr. and R. G. Elliman (Materials Research Society, Warrendale, PA); Mater. Res. Soc. Symp. Proc. 650 (2001) R3.18 $1-6$.

118. J. Tersoff, Phys. Rev. B39 (1989) 5566.

119. J. Tersoff, Phys. Rev. Lett. 64 (1990) 1757. 
120. H. Huang, N. Ghoniem, J. Wong and M. Baskes, Mater. Sci. Eng. 3 (1995) 615.

121. E. Pearson, T. Takai, T. Halicioglu and W. A. Tiller, "Computer modeling of Si and $\mathrm{SiC}$ surfaces and surface processes relevant to crystal growth from the vapor," $J$. Crystal Growth 70 (1984) 33-40.

122. M. I. Baskes, Phys. Rev. B46 (1992) 2727.

123. L. Malerba and J. M. Perlado, "Molecular dynamics simulation of irradiationinduced amorphization of cubic silicon carbide,” J. Nucl. Mater. 289 (2001) 57.

124. Xianglong Yuan and Linn W. Hobbs, "Modeling chemical and topological disorder in irradiation-amorphized silicon carbide," Nucl. Instrum. Meth. B 191 (2002) 7583.

125. A. Romano, M.-J. Caturla, J. Li. B. D. Wirth and S. Yip, "Defect-induced amorphization of $\beta$-SiC: a molecular dynamics study," to be submitted for publication (2002).

126. T. Diaz de la Rubia and M. W. Guinan, J. Nucl. Mater. 174 (1990) 151.

127. R. J. Price, J. Nucl. Mater. 33 (1969) 17.

128. J. M. Gibson and M. M. J. Treacy, "Diminished medium-range order observed in annealed amorphous germanium,” Phys. Rev. Lett. 78 (1997) 1074-77; M. M. J. Treacy and J. M. Gibson, Acta Cryst. A52 (1996) 212-20.

129. J. M. Gibson, M. M. J. Treacy and P. M. Voyles, Ultramicroscopy 83 (2000) 169.

130. L.-C. Qin and L. W. Hobbs, "Quantitative HREM of electron irradiated quartz," Mater. Res. Soc. Symp. Proc. 373 (1995) 329-34.

131. G. Y. Fan and J. M. Cowley, Ultramicroscopy 17 (1985) 345-56; 24 (1988) 49-60.

132. D. E. Jesson and S. J. Pennycook, Proc. Roy. Soc. London A441 (1993) 261-81.

133. E.J. Kirkland, Advanced Computing in Electron Microscopy (Plenum Press, New York, 1998) pp. 63-97.

134. S. J. Zinkle, L. L. Snead, W. S. Eatherly, J. W. Jones and D. K. Hensley, "Effect of low temperature ion irradiation on the microstructure of nitride ceramics," Mater. Res. Soc. Symp. Proc. 540 (1999) 305-10.

135. O. Chauvet, L. Zuppiroli, J. Ardonceau, I. Solomon, Y. C. Wang and R. F. Davis, "Paramagnetic defects in SiC-based materials,"Mater. Sci. Forum 83 (1992) 1201. 
136. D. G. Howitt, H. W. Cha, J. F. DeNatale and J. P. Heuer, J. Amer. Ceram. Soc. 74 (1991) 1145.

137. N. Umesaki, N. Hirosaki and K. Hirao, J. Non-Cryst. Solids 150 (1992) 120.

138. P. Vashishta, A. Nakano, R. K. Kalia nad I. Ebbsjö, J. Non-Cryst. Solids 182 (1995) 59.

139. P. Vashishta, R. K. Kalia, A. Nakano, W. Li and I. Ebbsjö, in: Amorphous Insulators and Semiconductors, ed. M. F. Thorpe and M. I. Mitkova (Kluwer Academic Publishers, Dordrecht, Netherlands, 1997) p. 151.

140. R. K. Kalia, A, Nakano, K. Tsuruta and P. Vashishta, Phys. Rev. Lett. 78 (1997) 689-92; R. K. Kalia, A. Nakano, A. Omeltchenko, K. Tsuruta and P. Vashishta, "Role of ultrafine microstructures in dynamic fracture in nanophase silicon nitride," Phys. Rev. Lett. 78 (1997) 2144-47.

141. F. de Brito Mota, J. F. Juston and A. Fazzio, "Structural properties of amorphous silicon nitride,” Phys. Rev. B58 (1998) 8323-28.

142. A. Zoroddu, F. Bernardini, P. Ruggerone and V. Fiorentini, "First-principles prediction of structure, energetics, formaion enthalpy, elastic constants, polarization and piezoelectric constants of AlN, GaN and InN: Comparison of local and gradientcorrected-density functional theory,” Phys. Rev. B64 (2001) 045208 1-6.

143. W. H. Zachariasen, J. Amer. Chem. Soc. 54 (1932) 3841.

144. C. H. L. Goodman, "Strained mixed-cluster mdoel for glass structure,” Nature 257 (1975) 370-72.

145. R. Hosemann, M. Hentschel, A. Lange, B. Uther and R. Brucknes, "Dreidimensional Analyse der parakristallen Struktur von Keiselglas,“ Z. Kristallogr. 169 (1984) 1333.

146. S. M. Nakhmanson, P. M. Voyles, M. Mousseau, G. T. Barkema and D. A. Drabold, “Realistic models of paracrystalline silicon,” Phys. Rev. B63 (2001) 235207 1-6.

147. C. S. Marians and J. K. Burdett, J. Non-Cryst. Solids 124 (19990) 1.

148. L. Guttman, J. Non-Cryst. Solids 116 (1990) 145.

149. K. Goetzke and H.-J. Klein, J. Non-Cryst. Solids 127 (1991) 215.

150. M. M. J. Treacy, J. M. Gibson and P. J. Keplinski, "Schläfli cluster topological analysis of medium range order in paracrystalline amorphous semiconductor models,” J. Non-Cryst. Solids 231 (1998) 99. 
151. M. M. J. Treacy, P. M. Voyles and J. M. Gibson, "Topological signatures of medium range order in amorphous semiconductor models," in: Amorphous and Heterogeneous Silcon Thin Films 2000, ed. H. M. Branz, R. W. Collins, S. Guha, H. Okamoto and M. Stutzmann, Mater. Res. Soc. Symp. Proc. 609 (2000) A2.5 1-6.

152. A. F. Wells, Further Studies of Three-Dimensional Nets, Monogrqph No. 8 (American Crystallographic Association, Polycrystal Book Service, Pittsburgh, PA, 1979).

153. M. O’Keefe, Z. Kristallogr. 196 (1991) 21.; M. O’Keeffe and B. G. Hyde, Crystal Structures: 1. Patterns and Symmetry, Monograph (Mineralogical Society of America, Washington, DC, 1996).

154. P. J. Burnett and G. A. D. Briggs, "The elastic properties of ion-implanted silicon," J. Mater. Sci. 21 (1986) 1828.

155. T. Diaz de la Rubia and G. H. Gilmer, "Structural transformations and defect production in ion implanted silicon: a molecular dynamics study," Phys. Rev. Lett. 74 (1995) 2507.

156. M.-J. Caturla, T. Diaz de la Rubia and G. H. Gilmer, "Ion beam processing of silicon at keV energies: a molecular dynamics study,” Phys. Rev. B54 (1996) 16683.

157. K. Nordlund, M. Ghaly, R. S. Averback, M. Caturla, T. Diaz de la Rubia and J. Tarus, "Defect production in collision cascades in elemental semiconductors and FCC metals,” Phys. Rev. B57 (1997) 7556.

158. J. Nord, K. Nordlund and J. Keinonen, "Amorphization mechanism and defect structures in ion amorphized Si, Ge and GaAs," Phys. Rev. B (2001) in press.

159. M. Chandrasekhar, H. R. Chandrasekhar, J. M. Meese and S. L. Thaler, "Effects of neutron irradiation on the Raman spectrum of silicon,” Inst. Phys. Conf. Ser. No. 59 (Institute of Physics, Bristol, UK, 1981) p. 205.

160. J. S. Custer, M. O Thompson, D. C. Jacobsen, J. M. Poate, S. Roorda, W. C. Sinke and F. Spaepen, "The density of amorphous Si," Mater. Res. Soc. Symp. Proc. 157 (1989) 689.

161. D. Franzblau and J. Tersoff, "Elastic properties of a network model of glasses," Phys. Rev. Lett. 68 (1992) 2172.

162. F. H. Stillinger and T. A. Weber, "Computer simulation of local order in condensed phases of silicon,” Phys. Rev. B31 (1985) 5262.

163. J. Tersoff, Phys. Rev. B38 (1988) 9902. 
164. J. F. Justo, M. Z. Bazant, E. Kaxiras, V. V. Bulatov and S. Yip, "Interatomic potential for silicon defects and disordered phases,” Phys. Rev. B58 (1998) 2539.

165. R. J. Keyse, A. J. Garratt-Reed, P. J. Goodhew and G. W. Lorimer, Introduction to Scanning Transmission Electron Microscopy (BIOS Scientific Publishers, Oxford, UK, 1998). 


\section{A1. PUBLICATIONS DURING GRANT PERIOD 1996-2002}

\section{Invited Book Chapters}

Linn W. Hobbs, C. Esther Jesurum and Bonnie Berger, "The topology of silica networks," Chapter 1 in: Structure and Imperfections in Amorphous and Crystalline Silica, ed. J.-P. Duraud, R. A. B. Devine and E. Dooryhee (John Wiley \& Sons, London, 2000) pp. 3-47.

L. W. Hobbs and X. Yuan, “Topology and topological disorder in silica," in: Defects in $\mathrm{SiO}_{2}$ and Related Dielectrics: Science and Technology, ed. G. Pacchioni, L. Skuja and D. Griscom (Kluwer, Dordrecht, The Netherlands, 2000) pp. 37-71.

\section{Refereed Journal and Conference Proceedings}

L. W. Hobbs, A. N. Sreeram, B. Berger and C. E. Jesurum, "Structural freedom, topological disorder, and the irradiation-induced amorphization of ceramics structures," [invited] Nucl. Instrum. Meth. Phys. Res. B 116 (1996) 17-25.

A. N. Sreeram, L. W. Hobbs, N. Bordes and R. C. Ewing, "Irradiation-induced amorphization of $\mathrm{AlPO}_{4}$,” Nucl. Instrum. Meth. Phys. Res. B 116 (1996) 126-30.

A. N. Sreeram, L.-C. Qin, A. J. Garratt-Reed and L. W. Hobbs, "Energy-filtered electron diffraction from amorphized solids in the dedicated scanning transmission electron microscope (STEM)," in: Microscopy \& Microanalysis 1996, ed. N. Zaluzek and G. W. Bailey (San Francisco Press, San Francisco, CA, 1996) pp. 702-03.

C. Esther Jesurum, Vinay Pulim, Bonnie A. Berger and Linn. W. Hobbs, "Topological modeling of topologically-disordered tetrahedral structures using local approaches," Materials Sci. Forum 239-241 (1997) 37-40.

A. J. Garratt-Reed, L.-C. Qin, A. N. Sreeram and L. W. Hobbs, "Measurement of radial density functions for glassy and metamict ceramics using energy-filtered electron diffrtaction in STEM," in: Electron Microscopy 1996 (European Fed. Electron Microscopy, Dublin, Ireland, 1997) pp. 23-4.

W. J. Weber, R. C. Ewing, C. A. Angell, G. W. Arnold, A. N. Cormack, J. M. Delaye, D. L. Griscom, L. W. Hobbs, A. Navrotsky, D. L. Price, A. M. Stoneham and M. C. Weinberg, [invited] "Radiation effects in glasses for high-level waste and plutonium disposition,” J. Mater. Res. 12 (1997) 1946-78. 
C. E. Jesurum, V. Pulim, B. Berger and L. W. Hobbs, "Modeling amorphization of tetrahedral structures using local approaches," in: Microstructure Evolution during Irradiation, eds. I. Robertson, G. Was, L. W. Hobbs and T. Diaz de la Rubia (Materials Research Society, Warrendale, PA, 1997); Mat. Res. Soc. Symp. Proc. 439 (1997) 63338.

D. C. Bell, A. J. Garratt-Reed and L. W. Hobbs, "Alternative collection mode for RDF acquisition in FEG-STEM,” Microscopy \& Microanalysis 3 [Suppl. 2] (1997) 1019-20.

C. E. Jesurum, V. Pulim and L. W. Hobbs, "Topological modeling of amorphized tetrahedral ceramic structures,” J. Nucl. Mater. 253 (1998) 87-103.

L. W. Hobbs, C. E. Jesurum, V. Pulim and B. Berger, "Local topology of silica networks," Philos. Mag. A78 (1998) 679-711.

C. E. Jesurum, V. Pulim and L. W. Hobbs, "Modeling the cascade amorphization of silicas with local-rules reconstruction,” Nucl. Instrum. Meth. B114 (1998) 25-34.

L. W. Hobbs, C. E. Jesurum and V. Pulim, "Topological modeling of cascade amorphization in network structures using local rules," Mater. Sci. Eng. A253 (1998) 1629.

W. J. Weber, R. C. Ewing, C. R. A. Catlow, T. Diaz de la Rubia, L. W. Hobbs, C. Kinoshita, Hj. Matzke, A. T. Motta, M. A. Nastasi, E. H. K. Salje, E. R. Vance and S. J. Zinkle, "Radiation effects in crystalline ceramic phases relevant to the immobilization and disposition of nuclear waste and weapons plutonium," J. Mater. Res.. 13 (1998) 1434-84.

C. E. Jesurum, V. Pulim, L. W. Hobbs and B. Berger, "Modeling collision cascade structures of $\mathrm{SiO}_{2}, \mathrm{Si}_{3} \mathrm{~N}_{4}$ and $\mathrm{SiC}$ using local topological approaches," Mat. Res. Soc. Symp. Proc. 504 (1998) 39-44.

D. C. Bell, A. J. Garratt-Reed and L. W. Hobbs, "RDF analysis of ion-amorphized $\mathrm{SiO}_{2}$ and $\mathrm{SiC}$ using post-specimen scanning in the field-emission scanning transmission electron microscope,” Mat. Res. Soc. Symp. Proc. 504 (1998) 147-52.

Linn W. Hobbs, C. Esther Jesurum and Vinay Pulim, "Local topological modeling of glass structure and radiation-induced arrangements in connected networks," [invited] in: Glass: Scientific Research for High-Performance Containment,” ed. E. Vernaz and O. Pinet (CEA/Valrhô, Bagnols-sur-Cèze, France, 1998), 16 pp.

David C. Bell, Anthony J. Garratt-Reed and Linn W. Hobbs, "RDF analysis of radiation amorphized SiC using field emission scanning electron microscopy," Microscopy \& Microanalysis 4 [Suppl. 2] (1998) 700-01. 
Linn W. Hobbs, C. Esther Jesurum and Bonnie Berger, "Rigidity constraints in the amorphization of singly- and multiply-polytopic structures,” [invited] in: Rigidity Theory and Applications, ed. P. M. Duxbury and M. F. Thorpe (Plenum Press, New York, 1999) pp. 191-216.

Linn W. Hobbs, C. Esther Jesurum and Bonnie Berger, "Rigidity constraints in amorphization of singly- and multiply-polytopic structures," [invited] in Microstructural Processes in Irradiated Materials, ed. S. J. Zinkle, G. Lucas, R. Ewing and J. Williams (Materials Research Society, Warrendale, PA, 1999); Mater. Res. Soc. Symp. Proc. 540 (1999) 717-28.

David C. Bell, Clark L. Allred and Linn W. Hobbs, "Local density information from low energy PEELS measurements,” in: Microscopy \& Microanalysis 5 [Supplement 2] (1999) 790-91.

Linn W. Hobbs, C. Esther Jesurum, Alexander Coventry, Vinay Pulim, Russell Schwartz and Bonnie Berger, "Towards a topological description of nearly crystalline networks," in: Advanced Materials for the 21st Century--Julia R. Weertman Symposium , ed. Y.-W. Chung, D. C. Dunand, P. K. Liaw and G. B. Olson (TMS, Warrendale, PA, 1999) pp. 475-86.

Xianglong Yuan, Vinay Pulim and Linn W. Hobbs, "Molecular dynamics refinement of topologically generated reconstructions of simulated irradiation cascades in silica networks," J. Nucl. Mater. 289 (2001) 71-79; “Erratum to 'Molecular dynamics refinement of topologically generated reconstruction of simulated irradiation cascades in silica networks,'” J. Nucl. Mater. 295 (2001) 132.

X. Yuan, V. Pulim and L. W. Hobbs, "Refinement of topologically modeled cascasdeamorphized silicas using molecular dynamics simulation," in Microstructural Processes in Irradiated Materials, ed. G. E. Lucas, L. Snead, M. A. Kirk, Jr. and R. G. Elliman (Materials Research Society, Warrendale, PA, 2001); Mat. Res. Soc. Symp. Proc. 650 (2001) R3.18 1-6.

X. Yuan and L. W. Hobbs, "Influence of interatomic potentials in MD investigation of ordering in a-SiC," in: Microstructural Processes in Irradiated Materials, ed. G. E. Lucas, L. Snead, M. A. Kirk ,Jr., and R. G. Elliman (Materials Research Society, Warrendale, PA, 2001); Mat. Res. Soc. Symp. Proc. 650 (2001) R3.45 1-7.

Michael Corradini, David Campbell, Micheline Draye, Charles Drummond III, Peter Hayward, Linn Hobbs, Edward Lahoda, Robin Rogers, Ben Sternberg and Edwin Zebroski, Research Needs for High-Level Waste Stored in Tanks and Bins at U.S. Department of Energy Sites (National Research Council, Washington, DC, 8 June 2001) 115 pp.

Steven T. Ostheim, Edgar Berkey, Charles H. Drummond III, Linn W. Hobbs, Pavel Hrma, Werner Lutze and Denis Strachan, Independent Evaluation of the Results of 
Additional Tests of the Radioactive Isolation Consortium, LLC (RIC) Advanced Vitrification System, Final Report, Task No. FT50503, DOE Contract No. DEAM26099FT40465 (Concurrent Technologies Corporation, Pittsburgh, PA, 12 July 2001) $37 \mathrm{pp}$.

Linn W. Hobbs, Xianglong Yuan, L. C. Qin, Vinay Pulim and Alexander Coventry, "The nanostructures of amorphous silicas," [invited] Microscopy \& Microanalysis 7 (2002) 29-34.

Xianglong Yuan and Linn W. Hobbs, "Modeling chemical and topological disorder in irradiation-amorphized silicon carbide,” Nucl. Instrum. Meth. Phys. Res. B 191 (2002) 75-83.

\section{Presentations}

Linn W. Hobbs, A. N. Sreeram and C. E. Jesurum, "Structural freedom, topological disorder, and the amorphization of ceramic structures," [Best Invited Paper Award, Eighth Int. Conf. on Radiation Effects in Insulators, Acriale Sicily, 9-15 September 1995] Nucl. Instrum. Meth. B 116 (1996) 17-25.

Linn W. Hobbs, $\underline{C}$. Esther Jesurum and Bonnie Berger, "Topological modeling of topologically-disordered tetrahedral structures using local approaches,” invited paper, 1996 Mardi Gras Conference on Experimental and Simulation Challenges in Nanostructured Materials, Baton Rouge, LA, 15-17 February 1996, unpublished.

Linn W. Hobbs, “On the Topological Stabilization of Amorphizable Structures," invited paper S3.02, Session on Stability and Floppy Modes in Glass, American Physical Society March Meeting, St. Louis, MO, 18-22 March 1996, unpublished.

L. W. Hobbs, C. E. Jesurum, B. A. Berger and V. Pulim, "Reconstruction and Deconstruction in Amorphization of Ceramics," invited paper, Symposium on Amorphization Mechanisms, American Ceramic Society 98th Annual Meeting, Indianapolis, IN, 15-17 April 1996; Amer. Ceram. Soc. Bull. 75 [3] (1996) 219.

Linn W. Hobbs, “Irradiation-Induced Rearrangements of Network Structures,” invited presentation, DOE Council on Materials Panel Workshop on Radiation Effects in Glasses for High-Level Waste and Plutonium Disposal, Sante Fe, NM, 25-29 February 1996; incorporated in: W. J. Weber, R. C. Ewing, C. A. Angell, G. W. Arnold, A. N. Cormack, J. M. Delaye, D. L. Griscom, L. W. Hobbs, A. Navrotsky, D . L. Price, A. M. Stoneham and M. C. Weinberg, "Radiation effects in glasses for high-level waste and plutonium disposition," J. Mater. Res. 12 (1997) 1946-78.

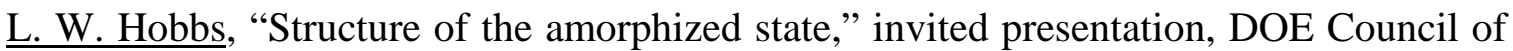
Materials Sciences Panel on Radiation Effects in Crystalline Ceramic Phases Relevant to the Disposition of Nuclear Waste and Weapons Plutonium, Lihue, Kaua'i, HI, 13-17 January 1997; incorporated in: W. J. Weber, R. C. Ewing, C. R. A. Catlow, T. Diaz de la Rubia, L. W. Hobbs, C. Kinoshita, Hj. Matzke, A. T. Motta, M. A. Nastasi, E. H. K. 
Salje, E. R. Vance and S. J. Zinkle, "Radiation effects in crystalline ceramic phases for the immobilization of high-level nuclear waste and plutonium,"J. Mater. Res. 13 (1998) 1434-84.

L. W. Hobbs, “Topological modeling of network structures,” invited paper, T. E. Mitchell Symposium on Defects in Crystals, Los Alamos, NM, 17-18 May 1997; published as: L. W. Hobbs, C. E. Jesurum, V. Pulim and B. Berger, "Local topology of silica networks," Philos. Mag. A 78 (1998) 679-711.

L. W. Hobbs, "Modeling radiation disorder in network structures using topological and local rules approaches," invited paper, Engineering Foundation Conference on Modification of Ceramics and Semiconductors by Ion Bombardment, Il Ciocco [Tuscany], Italy, 19-23 May 1997; published as: L. W. Hobbs, C. E. Jesurum and V. Pulim, "Topological modeling the cascade amorphization in network structures using local-rules" Mater. Sci. Eng. A253 (1998).

L. W. Hobbs, "Topological modeling of structure and disorder in network glasses using local rules," invited presentation, CEA Workshop on Glass: Scientific Research for High Performance Containment, Méjannes-le-Clap [Gard], France, 31 August-7 September 1997; published as: Linn W. Hobbs, C. Esther Jesurum and Vinay Pulim, "Local topological modeling of glass structure \& radiation-induced rearrangements in connected networks," in: Glass: Scientific Research for High Performance Containment, eds. E. Vernaz and O. Pinet (CEA/Valrhô, Bagnols-sur-Cèze, France, 1998).

L. W. Hobbs, "What can topological models tell us about glass structure and properties," Plenary Lecture, Joint Fall Meeting: American Ceramic Society, Glass \& Optical Materials Division; Optical Society of America Conference on Bragg Gratings; 12th International Conference on Optical Fiber Sensors, Williamsburg, VA, 25-28 October 1997; extended abstract published by Optical Soc. of America (1997).

C. E. Jesurum, L. W. Hobbs, V. Pulim and B. Berger, "Modeling collision cascade structure of $\mathrm{SiO}_{2}, \mathrm{Si}_{3} \mathrm{~N}_{4}$ and $\mathrm{SiC}$ using local topological approaches," Platform paper KK 1.6. Symposium on Atomistic Mechanisms in Beam Synthesis and Irradiation of Materials, Materials reseach Society Fall Meeting, Boston, MA, 1-5 December 1997

D. C. Bell, A. J. Garratt-Reed and L. W. Hobbs, "RDF analysis of ion-amorphized $\mathrm{SiO}_{2}$ and SiC using post-specimen scanning in the field-emission scanning transmission electron microscope," Poster paper KK 3.5, Symposium on Atomistic Mechanisms in Beam Synthesis and Irradiation of Materials, Materials Reseach Society Fall Meeting, Boston, MA, 1-5 December 1997; published in: Mat. Res. Soc. Symp. Proc. 504 (1998) 147-52.

L. W. Hobbs and C. M. Aldao, "Materials for Transportation/Energy," Invited discussion chair report, Workshop on Frontiers in Materials Research, Technology and Education, Rio de Janeiro, Brasil, 7-10 June 1998. 
Linn W. Hobbs, C. Esther Jesurum and Bonnie Berger, "Rigidity constraints in the amorphization of singly- and multiply-polytopic structures," invited paper, Workshop on Rigidity Theory and Applications, Traverse City, MI, 14-17 June 1998; published in: Rigidity Theory and Applications, ed. P. M. Duxbury and M. F. Thorpe (Plenum Press, New York, 1999) 191-216.

L. W. Hobbs, C. E. Jesurum, V. Pulim and B. Berger, "Modeling the structure of glass networks topologically uisng local rules algorithms," Platform paper ICG018-P02173XVII, International Congress on Glass, San Francisco, CA, 6-10 July 1998 (unpublished).

David C. Bell, Anthony J. Garratt-Reed and Linn W. Hobbs, "RDF analysis of radiation amorphized SiC using field emission scanning electron microscopy,” Microscopy \& Analysis '98, Atlanta, GA, 12-16 July 1998; extended abstract in: Microscopy \& Microanalysis 4 [Suppl. 2] (1998) 700-01.

Linn W. Hobbs, C. Esther Jesurum and Bonnie Berger, "Rigidity constraints in amorphization of singly and multiply-polytopic structures," paper N3.47, Symposium N, Microstructural Processes in Irradiated Materials, S. J. Zinkle, G. Lucas, R. Ewing and J. Williams, organizers, Materials Research Society Fall Meeting, Boston, MA, 30 November-2 December 1998; published in: Microstructural Processes in Irradiated Materials, ed. S. J. Zinkle, G. Lucas, R. Ewing and J. Williams (Materials Research Society, Warrendale, PA, 1999); Mater. Res. Soc. Symp. Proc. 540 (1999) 717-28.

Linn W. Hobbs, Alexander Coventry, Vinay Pulim, C. Esther Jesurum and Bonnie Berger, "Cascade disordering of silicas and other tetrahedral network structures using local topological modeling," invited paper N4.6, Symposium N, Microstructural Processes in Irradiated Materials, S. J. Zinkle, G. Lucas, R. Ewing and J. Williams, organizers, Materials Research Society Fall Meeting, Boston, MA, 30 November-2 December 1998 (unpublished).

A. Coventry, V. Pulim, C. E. Jesurum, B. Berger and L. W. Hobbs, "Structural modeling of aperiodic silicas using local rules self-assembly and graph theoretical optimization," paper S-B-039-99, $101^{\text {st }}$ Annual Meeting, American Ceramic Society, Indianapolis, IN, 25-28 April 1999 (unpublished).

Linn W. Hobbs, C. Esther Jesurum, Alexander Coventry, Vinay Pulim, Russell Schwartz and Bonnie Berger, "Towards a topological description of nearly crystalline networks," Advanced Materials for the 21st Century--Julia R. Weertman Symposium, TMS Annual Meeting, Cincinnati, OH, 31 October-4 November 1999; published in: Advanced Materials for the 21st Century--Julia R. Weertman Symposium, ed. Y.-W. Chung, D. C. Dunand, P. K. Liaw and G. B. Olson (TMS, Warrendale, PA, 1999) p. 475-86.

A. Coventry, V. Pulim, C. E. Jesurum, B. Berger and L. W. Hobbs, "Structural modeling of aperiodic tetrahedral networks using local rules self-assembly and graph theoretical 
optimization,” IXth International Conference on the Physics of Non-Crystalline Solids, Tucson, AZ, 17-21 October 1999 (unpublished).

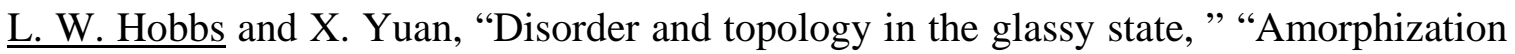
of crystalline silicas," "Modeling of topologically disordered silicas," "Defects in $\mathrm{SiO}_{2}$ : A Summary," four invited lectures, NATO Advanced Study Institute on Defects in $\mathrm{SiO}_{2}$ and Related Dielectrics: Science and Technology, Erice, Sicily, Italy, 8-20 April 2000; published in: Defects in $\mathrm{SiO}_{2}$ and Related Dielectrics, ed. G. Pacchioni, L. Skuja and D. L. Griscom (Kluwer Academic Publishers, Dordrecht, Netherlands, 2000) pp. 37-71.

L. W. Hobbs, L. C. Qin, V. Pulim and A. Coventry, "The nanostructures of amorphous silicas," invited paper, Sir John Meurig Thomas Symposium, Microscopy \& Microanalysis 2000, Philadelphia, PA, 13-17 August 2000; extended abstract in Microscopy \& Analysis 6 [Supplement. 2] (2000) 14-15; published in: Microscopy \& Microanalysis 7 (2001) in press.

Xianglong Yuan, Vinay Pulim and Linn W. Hobbs, "Molecular dynamics refinement of topologically generated reconstructions of simulated irradiation cascades in silica networks," paper B4-010-00, Symposium on Radiation Effects in Ceramics, 102nd Annual Meeting, American Ceramic Society, St. Louis, MO, April 2000; published in: J. Nucl. Mater. 289 (2001) 71-79; “Erratum,” J. Nucl. Mater. 295 (2001) 132.

X. Yuan, V. Pulim and L. W. Hobbs, "Refinement of topologically modeled cascasdeamorphized silicas using molecular dynamics simulation,” Paper. R3.18, Symposium R on Microstructural Processes in Irradiated Materials, Materials Research Society, Fall Meeting, Boston, MA, December 2000; published in: Microstructural Processes in Irradiated Materials, ed. G. E. Lucas, L. Snead, M. A. Kirk, Jr. and R. G. Elliman (Materials Research Society, Warrendale, PA, 2001); Mater. Res. Soc. Symp. Proc. 650 (2001) R3.18 1-7.

X. Yuan and L. W. Hobbs, "Influence of interatomic potentials in MD investigation of ordering in a-SiC," Paper R3.45, Symposium R on Microstructural Processes in Irradiated Materials, Materials Research Society fall meeting, Boston, MA, December 2000; published in: Microstructural Processes in Irradiated Materials, ed. G. E. Lucas, L. Snead, M. A. Kirk ,Jr., and R. G. Elliman (Materials Research Society, Warrendale, PA, 2001); Mater. Res. Soc. Symp. Proc. 650 (2001) R3.45 1-7.

X. Yuan and L. W. Hobbs, “Topologies of amorphous SiC,” paper B4F-02-2001-0, Symposium on Ceramic Science and Technology for the Nuclear Industry, $103^{\text {rd }}$ Annual Meeting, American Ceramic Society, Indianapolis, IN, 22-25 April 2001 (unpublished).

Linn W. Hobbs and Xianglong Yuan, "Molecular dynamics refinement of topologically simulated amorphizing irradiation cascades in silica and silicon carbide," invited paper, Symposium on Radiation Effects and Nuclear Waste Immobilization, Cambridge, UK, 3031 August 2001 (unpublished). 
Xianglong Yuan and Linn W. Hobbs, "Molecular dynamics refinement of topologically simulated irradiation cascades in silica," Paper 01.6, $11^{\text {th }}$ International Congress on Radiation Effects in Insulators (REI-11), Lisbon, Portugal, 3 September 2001 (unpublished).

Xianglong Yuan and Linn W. Hobbs, "Modeling chemical and topological disorder in irradiation-amorphized silicon carbide," Paper P-A.20, $11^{\text {th }}$ International Congress on Radiation Effects in Insulators (REI-11), Lisbon, Portugal, 3 September 2001; published in: Nucl. Instrum. Meth. Phys. Res. B 191 (2002) 75-83. 